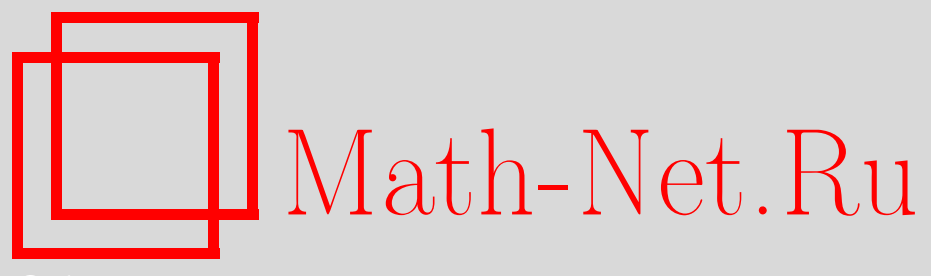

А. В. Борисов, И. С. Мамаев, А. В. Цыганов, Неголономная динамика и пуассонова геометрия, УМН, 2014, том 69, выпуск 3, 87-144

DOI: https://doi.org/10.4213/rm9587

Использование Общероссийского математического портала Math-Net.Ru подразумевает, что вы прочитали и согласны с пользовательским соглашением http://www . mathnet.ru/rus/agreement

Параметры загрузки:

IP : 54.92 .164 .108

26 апреля 2023 г., 02:39:58

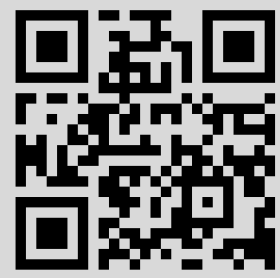




\title{
Неголономная динамика и пуассонова геометрия
}

\begin{abstract}
А. В. Борисов, И. С. Мамаев, А. В. Цыганов
В работе собраны основные известные на настоящее время сведения о нелинейных пуассоновых структурах, возникающих в неголономной механике при рассмотрении интегрируемых систем. Доказано, что использование теории пуассоновых деформаций позволяет привести различные неголономные системы к динамическим системам на хорошо изученных фазовых пространствах с линейными скобками Ли-Пуассона. В результате мы можем не только сравнивать различные неголономные интегрируемые системы друг с другом, но и использовать для их изучения достаточно развитые методы пуассоновой геометрии и топологии.
\end{abstract}

Библиография: 95 названий.

Ключевые слова: неголономные системы, скобки Пуассона, шар Чаплыгина, система Суслова, система Веселовой.

DOI: $10.4213 / \mathrm{rm} 9587$

\section{СОДЕРЖАНИЕ}

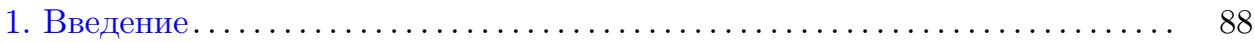

1.1. Основные определения . . . . . . . . . . . . . . . . . . . 90

1.2. Скобки $e(3)$ и пуассоновы структуры ранга $4 \ldots \ldots \ldots \ldots \ldots \ldots . . \ldots 2$

1.3. Геометрическая формулировка метода приводящего множителя 95

1.4. Приведение скобок Пуассона к каноническим ............... 99

2. Движение твердого тела по плоскости ...................... 102

2.1. Шар Чаплыгина ...................................... 104

2.2. Конформно-гамильтоново представление . . . . . . . . . . . . . 105

2.3. Приведение скобок Пуассона к каноническим .............. 106

2.4. Гиростатические обобщения ........................ 108

3. Шар Чаплыгина на сфере - БМФ-система...................... 109

3.1. Скобки Пуассона и конформно-гамильтоново представление... . 109

3.2. Приведение скобок Пуассона к каноническим .............. 111

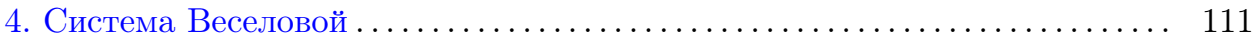

4.1. Эквивалентность системы Веселовой и шара Чаплыгина......... 112

4.2. Гиростатическое обобщение ...................... 114

Работа первого автора выполнена в рамках государственного задания УдГУ “Регулярная и хаотическая динамика". Работа второго автора поддержана грантом Российского научного фонда № 14-19-01303 “Динамика и управление мобильных робототехнических систем”.

(С) А. В. Борисов, И. С. Мамаев, А. В. ЦыГАнов, 2014 
5. Тело вращения на плоскости . ............................ 115

5.1. Гамильтонизация редуцированной системы ............... 118

5.2. Пуассонова структура ранга $4 \ldots \ldots \ldots \ldots \ldots \ldots \ldots \ldots \ldots \ldots \ldots . \ldots \ldots$

5.3. Приведение скобок Пуассона к каноническим ............... 122

5.4. Гиростатические обобщения ......................... 123

5.5. Сфера Рауса ................................... 124

6. Динамически симметричный шар на поверхности вращения .......... 127

6.1. Скобки Пуассона для шара на поверхности вращения.......... 130

6.2. Гамильтонизация на редуцированном подмногообразии ........ . 132

6.3. Приведение скобок Пуассона к каноническим ................ 133

6.4. Движение по параболоиду вращения ................... 134

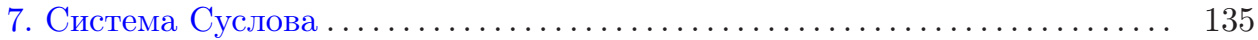

7.1. Пуассоновы структуры при движении по инерции............ 136

7.2. Частный случай движения в потенциальном поле ............ 138

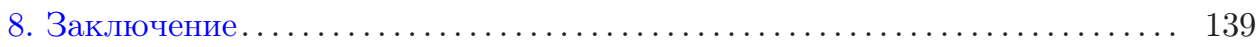

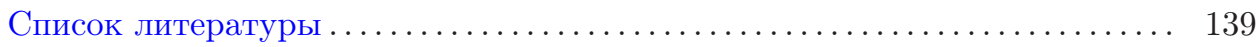

\section{1. Введение}

В обзоре рассматривается ряд возникающих в неголономной механике динамических систем, допускающих описание с помощью методов пуассоновой геометрии [21]. Не вдаваясь в подробности, отметим, что неголономная механика и неголономная геометрия возникли в конце XIX-начале XX в. и в настоящее время наблюдается устойчивый рост новых исследований в этой области (см. [6], [10], [12], [16]-[19], [22]-[24], [26], [27], [36], [39]-[41], [45], [73], [78], [81]-[85]).

Остановимся подробнее на различии между неголономной механикой и неголономной геометрией. В обоих случаях мы имеем дело с некоторыми дифференциальными уравнениями, обобщающими известные уравнения Лагранжа и Гамильтона стандартной классической механики. Однако уравнения неголономной механики постулируются из уравнений идеальности неинтегрируемых (неголономных) связей и выводятся из принципа Даламбера-Лагранжа, а в неголономной геометрии для получения дифференциальных уравнений используется вариационный принцип Гамильтона с учетом связей (хотя, например, в старых работах (см., например, [86]) под неголономной геометрией понимается именно неголономная механика).

Неголономная механика используется для описания динамики систем с качением (где неголономная связь выражает условие непроскальзывания - скорость точки контакта равна нулю). Неголономная геометрия, называемая также субримановой (современное введение в нее см. в [10], [51], [52], [64], [88]-[91]), может применяться для исследования задач оптимального управления (см. [1]), а также для описания динамики реальных систем гидродинамического происхождения (систем с сильной анизотропией тензора инерции). Последняя область исследований получила название вакономной механики, с ней можно ознакомиться по серии работ, указанных в [7]. Отметим также работу [8], в которой 
открыто однопараметрическое семейство моделей систем со связями, “соединяющее" неголономную геометрию и классическую неголономную механику. В работе [11] получены объединяющие вакономные уравнения для неголономных систем, которые могут быть использованы для оптимального управления систем с качением (например, робота-шара).

Различие между неголономной механикой и неголономной геометрией (вакономной механикой) иногда игнорируется, что приводит к выводам, противоречащим принципам механики (см., например, [43], где для описания качения диска по плоскости используются не неголономные, а вакономные уравнения). Отметим, однако, что некоторые траектории для неголономной и вакономной моделей могут совпадать [59]. Уравнения неголономной (субримановой) геометрии заведомо являются гамильтоновыми, однако имеют вырожденный по импульсам гамильтониан, что приводит к новым геометрическим конструкциям (абнормальные экстремали и пр.). Уравнения неголономной механики в общем случае не являются гамильтоновыми и содержат неустранимые члены неголономности. Однако при дополнительных симметриях задачи (что в механике достигается при специальных значениях параметров, описывающих динамические и геометрические свойства системы) возможно получить представление системы в гамильтоновой и конформно-гамильтоновой формах. В зависимости от параметров системы возникает иерархия динамического поведения неголономных систем, впервые предложенная в [23]. Возникающие при этом скобки Пуассона могут иметь весьма нетривиальную и нелинейную структуру и представляют самостоятельный интерес для общей теории пуассоновых структур [21]. Альтернативная точка зрения развивается в обзоре [36] (см. также библиографию к нему), где для описания неголономных систем используется почти пуассонов подход, состоящий в постулировании некоторой скобки типа Пуассона, которая, однако, не удовлетворяет тождеству Якоби. Отметим, однако, что этот подход пока не привел к содержательным результатам.

Наш подход развивает идею иерархии динамического поведения неголономных систем и связан с анализом тензорных инвариантов уравнений движения. По существу, он впервые был намечен В. В. Козловым [53], [55] и систематически развит в работах [23], [24], [26], [27]. К тензорным инвариантам относятся: скалярные функции - первые интегралы, а также поля симметрий, пуассоновы структуры (как правило - вырожденные), инвариантные меры. Их существование для неголономных систем имеет скрытый (не имеющий очевидного симметрийного происхождения) характер. Отметим, что еще в XIX в. (Г. Герц, А. Пуанкаре) было отмечено, что для неголономных систем не применим вариационный принцип Гамильтона, лишь позже (С. А. Чаплыгин [29], см. также [58]) стало понятно, что теорема, аналогичная теореме Нётер, может быть обобщена на неголономные системы при некоторых естественных дополнительных ограничениях. Поиск и исследование скрытых тензорных инвариантов стали возможными после появления мощных систем аналитических вычислений.

Применение этого подхода к задаче о неголономном качении твердого тела по плоскости и сфере было проиллюстрировано в работах [23], [24], [26], [27]. В них было введено понятие конформно-гамильтоновых систем, которые становятся гамильтоновыми после соответствующей “хорошей” замены времени. 
Это приводит к проблеме гамильтонизации неголономных систем, которая заключается в нахождении необходимого и достаточного числа тензорных инвариантов, позволяющих записать систему в конформно-гамильтоновом виде. Некоторые части этих исследований (тесно связанные с существованием инвариантных мер и интегрируемостью) пересекаются с более ранними результатами А. П. Веселова, Л. Е. Веселовой и Ю. И. Федорова. С аналитической точки зрения проблема гамильтонизации рассмотрена в работах [12], [54], [56], в этом случае в ней выделяются "полулокальный" (в окрестности инвариантного многообразия) и глобальный аспекты.

В данном обзоре, предназначенном в основном для математиков, мы не будем останавливаться на выводе уравнений движения и физических принципах неголономной механики, отсылая читателя к прекрасному обзору [5].

Мы рассматриваем в основном классические постановки задач, восходящие к Э. Раусу, П. Аппелю, С. А. Чаплыгину, Г. К. Суслову и В. Вагнеру. Они описывают механические системы, связанные с качением тел без проскальзывания (это условие и приводит к неголономной связи), уравнения движения для них хорошо известны. Для этих систем мы систематизируем известные результаты и приводим ряд новых результатов, связанных с приведением к пуассоновой (гамильтоновой) форме, а также обсуждаем явления, препятствующие такому приведению. В некоторых случаях явно удается найти лишь конформно-гамильтоново разложение, и в этих случаях еще предстоит выяснить, какие результаты пуассоновой геометрии и динамики могут быть обобщены на подобные системы. Как было отмечено в [25], [26], неголономная динамика не только интересна с точки зрения приложения хорошо развитого аппарата гамильтоновой динамики, но и генерирует ряд содержательных теоретических вопросов в области, где система очень близка к гамильтоновой. На этом пути возникает замечательная взаимосвязь с теорией обратимых систем, теорией инвариантных мер, а также новых нелинейных пуассоновых структур (поведение симплектических листов которых может иметь хаотический характер), представляющих большой общематематический интерес.

1.1. Основные определения. Динамическая система на фазовом пространстве $\mathscr{M}, \operatorname{dim} \mathscr{M}=n$, с координатами $\left(x_{1}, \ldots, x_{n}\right)=x$ определяется уравнениями движения

$$
\dot{x}_{i}=X_{i}(x), \quad i=1, \ldots, n .
$$

Эта система дифференциальных уравнений задает векторное поле

$$
X=\sum_{i=1}^{n} X_{i} \frac{\partial}{\partial x_{i}},
$$

которое является линейным оператором на пространстве гладких функций на многообразии $\mathscr{M}$, определяющим эволюцию любой гладкой функции $F(x)$ :

$$
\dot{F}=X(F)=\sum_{i=1}^{n} X_{i} \frac{\partial F}{\partial x_{i}} .
$$

Для гамильтоновой системы векторное поле

$$
X=P \mathrm{~d} H
$$


определяется с помощью функиии Гамильтона $H(x)$ и бивектора, или тензора, Пуассона $P(x)$, задающего скобки Пуассона на фазовом пространстве $\mathscr{M}$ по правилу

$$
\{f, g\}_{P}=\sum_{i, j=1}^{n} P_{i j}(x) \frac{\partial f}{\partial x_{i}} \frac{\partial g}{\partial x_{j}} .
$$

В дальнейшем, если это не вызовет недоразумений, индекс $P$ у скобок мы будем опускать.

Так как скобка Пуассона билинейна, кососимметрична и удовлетворяет тождеству Якоби, то для коэффициентов $P$ выполнены соотношения

$$
P_{i j}=-P_{j i}, \quad \sum_{m}\left(P_{i m} \frac{\partial P_{j k}}{\partial x_{m}}+P_{j m} \frac{\partial P_{k i}}{\partial x_{m}}+P_{k m} \frac{\partial P_{i j}}{\partial x_{m}}\right)=0,
$$

где $i, j, k=1, \ldots, n$. Как известно [60], кратко эти условия можно переписать с помощью скобки Схоутена $[\cdot, \cdot]$ в виде

$$
[P, P]=0 .
$$

Напомним, что скобка Схоутена $[A, B]$ бивекторов $A$ и $B$ является тривектором, элементы которого в локальных координатах $x$ на многообразии $\mathscr{M}$ имеют вид

$$
[A, B]_{i j k}=-\sum_{m=1}^{\operatorname{dim} \mathscr{M}}\left(B_{m k} \frac{\partial A_{i j}}{\partial x_{m}}+A_{m k} \frac{\partial B_{i j}}{\partial x_{m}}+\operatorname{cycle}(i, j, k)\right) .
$$

Наиболее известными бивекторами Пуассона являются:

- постоянный бивектор - каноническая скобка Пуассона:

$$
P=\left(\begin{array}{cc}
0 & I \\
-I & 0
\end{array}\right), \quad\{f, g\}=\sum_{i=1}^{m}\left(\frac{\partial f}{\partial q_{i}} \frac{\partial g}{\partial p_{i}}-\frac{\partial f}{\partial p_{i}} \frac{\partial g}{\partial q_{i}}\right), \quad x=(q, p)
$$

- линейный бивектор - скобка Ли-Пуассона:

$$
P_{i j}=\sum_{k=1}^{n} G_{i j}^{k} x_{k}, \quad\left\{x_{i}, x_{j}\right\}=\sum_{k=1}^{n} G_{i j}^{k} x_{k},
$$

где $G_{i j}^{k}$ - структурные константы алгебры Ли. Подробное руководство, посвященное пуассоновым структурам в гамильтоновой динамике, и подробная библиография содержатся в [21].

Естественным обобщением гамильтоновых систем являются конформно-гамильтоновы системы, для которых векторное поле представляется в виде

$$
X=g(x) P \mathrm{~d} H
$$

где $P$ - тензор Пуассона, а функция $g(x)$ - приводящий множитель - как правило, полагается положительно определенной [6], [63].

В некоторых случаях, даже если теоретически доказано, что система является гамильтоновой или конформно-гамильтоновой, явно найти представление (3) или (7) не удается. Поэтому рассмотрим более общие системы, обладающие набором первых интегралов $H_{1}(x), \ldots, H_{m}(x)$, для которых векторное 
поле представляется с помощью тензора Пуассона, обладающего заданными свойствами:

$$
\begin{gathered}
X=g_{1}(x) P \mathrm{~d} H_{1}+\cdots+g_{m}(x) P \mathrm{~d} H_{m}, \\
{[P, P]=0, \quad\left\{H_{i}, H_{j}\right\}_{P}=0 \quad \forall i, j .}
\end{gathered}
$$

В дальнейшем будем называть это представление разложением по конформно-гамильтоновым полям (или конформно-гамильтоновым разложением).

При этом, конечно, возможность использования такого представления для изучения динамики зависит от конкретного вида коэффициентов разложения $g_{i}$, которые являются функциями на всем фазовом пространстве.

ЗАмЕчАНИЕ 1. Для многих априори негамильтоновых систем, обладающих первым интегралом $H$, можно записать исходное векторное поле $X$ в кососимметричной форме

$$
X=\widehat{P} \mathrm{~d} H,
$$

если ввести подходящее кососимметрическое тензорное $(2,0)$-поле $\widehat{P}$, которое в общем случае не удовлетворяет тождеству Якоби. Такое представление иногда называют почти гамильтоновым [45], [46], [62], [87]. Оно встречается, например, в неголономной механике. Однако при этом приходится отказываться и от всех методов исследования, разработанных в симплектической (пуассоновой) геометрии и топологии. Например, необходимо отказаться от отождествления поверхностей уровня интегралов движения с лагранжевыми подмногообразиями.

Целью данной работы является представление различных систем неголономной механики в одной из возможных форм (3), (7) или (8). Возникающие на этом пути нелинейные бивекторы Пуассона являются, как правило, деформациями скобки Ли-Пуассона алгебры $e(3)$. Используя теорию пуассоновых деформаций, мы можем привести различные неголономные системы к динамическим системам на хорошо изученных фазовых пространствах с канонической скобкой Пуассона. Это позволяет сравнивать различные неголономные интегрируемые системы друг с другом и использовать для их изучения достаточно развитые методы пуассоновой геометрии и топологии [4], [91].

1.2. Скобки $e(3)$ и пуассоновы структуры ранга 4. Для большинства рассматриваемых ниже систем фазовое пространство оказывается диффеоморфным четырехмерному многообразию $T^{*} S^{2}$. Оно, как известно, допускает стандартное вложение в $\mathbb{R}^{6}$, обладающее пуассоновой структурой, отвечающей алгебре $e(3)$ группы движений $E(3)$. Напомним, что эти скобки Ли-Пуассона имеют вид

$$
\left\{L_{i}, L_{j}\right\}_{0}=\varepsilon_{i j k} L_{k}, \quad\left\{L_{i}, \gamma_{j}\right\}_{0}=\varepsilon_{i j k} \gamma_{k}, \quad\left\{\gamma_{i}, \gamma_{j}\right\}_{0}=0
$$

где $\varepsilon_{i j k}$ - полностью антисимметрический тензор. Соответствующий канонический бивектор Пуассона на алгебре $e^{*}(3)$ равен

$$
P_{0}=\left(\begin{array}{ll}
0 & \boldsymbol{\Gamma} \\
\boldsymbol{\Gamma} & \mathbf{L}
\end{array}\right) .
$$


В это определение входят кососимметрические $(3 \times 3)$-матрицы

$$
\boldsymbol{\Gamma}=\left(\begin{array}{ccc}
0 & \gamma_{3} & -\gamma_{2} \\
-\gamma_{3} & 0 & \gamma_{1} \\
\gamma_{2} & -\gamma_{1} & 0
\end{array}\right), \quad \mathbf{L}=\left(\begin{array}{ccc}
0 & L_{3} & -L_{2} \\
-L_{3} & 0 & L_{1} \\
L_{2} & -L_{1} & 0
\end{array}\right)
$$

используемые для описания стандартного изоморфизма

$$
z=\left(\begin{array}{c}
z_{1} \\
z_{2} \\
z_{3}
\end{array}\right) \rightarrow \mathbf{Z}=\left(\begin{array}{ccc}
0 & z_{3} & -z_{2} \\
-z_{3} & 0 & z_{1} \\
z_{2} & -z_{1} & 0
\end{array}\right)
$$

двух трехмерных алгебр Ли $\left(\mathbb{R}^{3}, a \times b\right)$ и $(\mathrm{so}(3),[a, b])$ с векторным произведением и матричным коммутатором.

Напомним, что скобки Пуассона (9) обладают двумя функциями Казимира

$$
C_{1}=(\gamma, \gamma), \quad C_{2}=(\gamma, L), \quad P_{0} \mathrm{~d} C_{i}=0, \quad i=1,2,
$$

и что при нулевом значении функции Казимира $C_{2}=0$ симплектические листы канонического бивектора Пуассона $P_{0}$ симплектоморфны кокасательным пространствам $T^{*} S^{2}$ двумерной сферы.

ЗАмЕчаниЕ 2. Практически все полученные нами ниже скобки Пуассона (в том числе нелинейные) приводятся к скобкам Ли-Пуассона алгебры $e(3)$.

Для динамической системы, интегрируемой по теореме Эйлера-Якоби о последнем множителе, описание поведения в окрестности компактных интегральных многообразий

$$
\boldsymbol{\Sigma}_{h}^{2}=\left\{x \in \mathscr{M}: H_{1}(x)=h_{1}, \ldots, H_{n-2}(x)=h_{n-2}\right\}, \quad h_{k} \in \mathbb{C},
$$

опирается на классические результаты Пуанкаре, Зигеля, Колмогорова и др. о динамических системах на двумерном торе (см., например, [5], [12], [57]).

Напомним, что если связная компонента уровня интегралов движения диффеоморфна двумерному тору и на этом торе мы имеем динамическую систему с инвариантной мерой, то, согласно теореме Колмогорова [49], можно ввести координаты $\varphi_{1}, \varphi_{2}(\bmod 2 \pi)$, в которых эта система принимает вид

$$
\dot{\varphi}_{1}=\frac{c_{1}}{\Phi}, \quad \dot{\varphi}_{2}=\frac{c_{2}}{\Phi} .
$$

Здесь $\Phi$ - гладкая положительная функция на торе, а $c_{1,2}$ - некоторые константы. Замена времени $\mathrm{d} t \rightarrow \Phi \mathrm{d} \tau$ позволяет линеаризовать уравнения движения, и поэтому движение происходит по прямолинейным обмоткам тора, хотя, возможно, и не равномерно [53].

В окрестности неособого тора после замены времени $\Phi^{-1} \mathrm{~d} t \rightarrow \mathrm{d} \tau$ угловые переменные $\varphi_{1}, \varphi_{2}$ могут быть дополнены каноническими переменными действия $I_{1}, I_{2}[12]$, поэтому векторное поле (11) является конформно-гамильтоновым

$$
X=g_{1} P \mathrm{~d} H_{1}, \quad g_{1}=\Phi^{-1},
$$


относительно канонического бивектора Пуассона ранга четыре

$$
P=\left(\begin{array}{cccc}
0 & 0 & 1 & 0 \\
0 & 0 & 0 & 1 \\
-1 & 0 & 0 & 0 \\
0 & -1 & 0 & 0
\end{array}\right)
$$

Отметим, однако, что конструктивного алгоритма нахождения пуассоновой структуры и соответствующего ему конформно-гамильтонова представления поля $X$ в терминах исходных физических переменных в общем случае до сих пор не разработано. Одним из подобных конструктивных методов является метод приводящего множителя Чапльгина, который позволяет эффективно находить скобки Пуассона на полностью приведенном фазовом пространстве $\mathscr{M} / G[24]$.

В случае частично приведенного пространства $\mathscr{M} / G_{1}$ мы будем использовать метод поиска скобок Пуассона, предложенный в работах [81]-[83]. С математической точки зрения основная идея данного подхода состоит в отождествлении совместных поверхностей уровня интегралов движения с лагранжевым слоением симплектических листов искомой пуассоновой структуры.

Действительно, если векторное поле $X(1)$ на $n$-мерном фазовом пространстве обладает $n-2$ интегралами движения $H_{1}, \ldots, H_{n-2}$ и интегральным инвариантом (например, инвариантной мерой), то данное векторное поле интегрируемо в квадратурах согласно теореме Эйлера-Якоби. Мы будем предполагать, что поверхности уровня интегралов являются лагранжевыми поверхностями на симплектических листах некоторого бивектора Пуассона $P$, точно так же, как и в случае интегрируемости гамильтоновых систем по Лиувиллю. Так как размерность лагранжева подмногообразия равна половине размерности симплектического листа, то мы предполагаем, что

$$
\operatorname{rank} P=4
$$

почти всюду. Примеры скобок второго ранга могут быть найдены в рабо$\operatorname{Tax}[37],[66]$.

ЗАмечАниЕ 3. Точки пуассонова многообразия, где $\operatorname{rank} P$ максимален (в нашем случае равен 4), называются регулярными, а точки, где rank $P$ меньше максимального, называются сингулярными. С точки зрения динамики сингулярные точки обычно совпадают с положениями равновесия (устойчивые равновесия) и в этих точках возникают симплектические листы меньших размерностей (тонкие орбиты) и дополнительные функции Казимира.

Для большинства рассматриваемых далее примеров полностью или частично редуцированное фазовое пространство будет шестимерным, $\operatorname{dim} \mathscr{M} / G=6$, с локальными координатами $\left(\gamma_{1}, \gamma_{2}, \gamma_{3}, M_{1}, M_{2}, M_{3}\right)$. Интегрируемое по Эйлеру-Якоби векторное поле $X$ на этом многообразии обладает четырьмя интегралами движения $H_{1}, \ldots, H_{4}$ и инвариантной мерой.

Для того чтобы построить искомый бивектор $P$, нам необходимо решить следующую систему уравнений:

$$
[P, P]=0, \quad\left\{H_{k}, H_{m}\right\}=0, \quad k, m=1, \ldots, 4,
$$


где $[\cdot, \cdot \cdot]$ - скобки Схоутена. Основная сложность при этом заключается в том, что система уравнений (12) имеет априори бесконечное число решений [79]. Поэтому мы ограничимся рассмотрением линейных по переменным $M_{1}, M_{2}$ и $M_{3}$ бивекторов $P$ :

$$
P_{i j}=\sum_{k=1}^{3} c_{i j}^{k}(\gamma) M_{k}+d_{i j}(\gamma),
$$

где $c_{i j}^{k}$ и $d_{i j}$ - неизвестные функции от $\gamma$. Это сужает пространство поиска решений уравнений (12) и позволяет найти частные решения данных уравнений.

Заметим, что во многих случаях при поиске пуассоновой структуры среди первых интегралов $H_{1}, \ldots, H_{4}$ по физическим соображениям можно выбрать пару функций Казимира, например, $\gamma^{2}$ и $(M, \gamma)$ в задаче о шаре Чаплыгина [22]. Здесь мы отказываемся от этого и по аналогии с поиском бигамильтоновой структуры в задаче о волчке Лагранжа [80] считаем, что все интегралы $H_{1}, \ldots, H_{4}$ равноправны.

\section{3. Геометрическая формулировка метода приводящего множи- теля.}

Обобщенные системы Чаплыгина. Напомним, что согласно [24] обобщенной системой Чаплыгина называется механическая система с двумя степенями свободы, уравнения движения которой можно записать в форме

$$
\begin{gathered}
\frac{\mathrm{d}}{\mathrm{d} t}\left(\frac{\partial L}{\partial \dot{q}_{1}}\right)-\frac{\partial L}{\partial q_{1}}=\dot{q}_{2} S, \quad \frac{\mathrm{d}}{\mathrm{d} t}\left(\frac{\partial L}{\partial \dot{q}_{2}}\right)-\frac{\partial L}{\partial q_{2}}=-\dot{q}_{1} S \\
S=a_{1}(q) \dot{q}_{1}+a_{2}(q) \dot{q}_{2}+b(q)
\end{gathered}
$$

где $L$ - функция обобщенных координат $q=\left(q_{1}, q_{2}\right)$ и скоростей $\dot{q}=\left(\dot{q}_{1}, \dot{q}_{2}\right)$, которую также будем называть лагранжианом системы. Как несложно проверить, эта система допускает интеграл энергии обычного вида

$$
E=\sum_{i} \frac{\partial L}{\partial \dot{q}_{i}} \dot{q}_{i}-L
$$

ЗАмечАниЕ 4. Обычная система Чаплыгина получается при специальном выборе функции $S$ (при этом заведомо $b(q)=0$ ) [32]. В работах [28], [48], [76] предложено несколько иное обобщение систем Чаплыгина.

Известно, что если имеется инвариантная мера с плотностью, зависящей только от координат, то система представляется в конформно-гамильтоновой форме [24] (при условии $b(q)=0$ это было доказано самим С. А. Чаплыгиным [32]). Чтобы показать это, выполним преобразование Лежандра для исходной системы (14):

$$
P_{i}=\frac{\partial L}{\partial \dot{q}_{i}}, \quad H=\left.\left(\sum_{i} P_{i} \dot{q}_{i}-L\right)\right|_{\dot{q}_{i} \rightarrow P_{i}},
$$

при этом уравнения движения (14) перепишутся в виде

$$
\begin{aligned}
\dot{q}_{i} & =\frac{\partial H}{\partial P_{i}}, \quad \dot{P}_{1}=-\frac{\partial H}{\partial q_{1}}+\frac{\partial H}{\partial P_{2}} S, \quad \dot{P}_{2}=-\frac{\partial H}{\partial q_{2}}-\frac{\partial H}{\partial P_{1}} S, \\
S & =a_{1}(q) \dot{q}_{1}+a_{2}(q) \dot{q}_{2}+b(q)=A_{1}(q) P_{1}+A_{2}(q) P_{2}+B(q) .
\end{aligned}
$$


Здесь $H$ совпадает с интегралом энергии (15), выраженным через новые переменные.

Предположим теперь, что система допускает инвариантную меру с плотностью, зависящей лишь от координат:

$$
\mu=\mathscr{N}(q) \mathrm{d} P_{1} \mathrm{~d} P_{2} \mathrm{~d} q_{1} \mathrm{~d} q_{2} .
$$

В этом случае уравнение Лиувилля для $\mathscr{N}(q)$ приводится к виду

$$
\dot{q}_{1}\left(\frac{1}{\mathscr{N}} \frac{\partial \mathscr{N}}{\partial q_{1}}-A_{2}(q)\right)+\dot{q}_{2}\left(\frac{1}{\mathscr{N}} \frac{\partial \mathscr{N}}{\partial q_{2}}+A_{1}(q)\right)=0,
$$

и поскольку $\mathscr{N}$ зависит лишь от координат, каждая из скобок должна обращаться в нуль по отдельности:

$$
\frac{1}{\mathscr{N}} \frac{\partial \mathscr{N}}{\partial q_{1}}-A_{2}(q)=0, \quad \frac{1}{\mathscr{N}} \frac{\partial \mathscr{N}}{\partial q_{2}}+A_{1}(q)=0 .
$$

Условие локальной разрешимости системы уравнений (18) имеет вид

$$
\frac{\partial A_{1}}{\partial q_{1}}+\frac{\partial A_{2}}{\partial q_{2}}=0
$$

После замены переменных

$$
P_{i}=\frac{p_{i}}{\mathscr{N}(q)}, \quad i=1,2,
$$

справедливы следующие соотношения для производных:

$$
\frac{\partial H}{\partial P_{i}}=\mathscr{N} \frac{\partial \bar{H}}{\partial p_{i}}, \quad \frac{\partial H}{\partial q_{i}}=\frac{\partial \bar{H}}{\partial q_{i}}+\frac{1}{\mathscr{N}} \frac{\partial N}{\partial q_{i}}\left(\frac{\partial \bar{H}}{\partial p_{1}} p_{1}+\frac{\partial \bar{H}}{\partial p_{2}} p_{2}\right) .
$$

Здесь $\bar{H}(q, p)=H(q, P(q, p))$ - гамильтониан в новых переменных.

Подставляя полученные соотношения в (16) и пользуясь соотношениями (18), получим

$$
\begin{aligned}
\dot{q}_{i} & =\mathscr{N}(q) \frac{\partial \bar{H}}{\partial p_{i}} \\
\dot{p}_{1} & =\mathscr{N}(q)\left(-\frac{\partial \bar{H}}{\partial q_{1}}+\mathscr{N}(q) B(q) \frac{\partial \bar{H}}{\partial p_{2}}\right), \\
\dot{p}_{2} & =\mathscr{N}(q)\left(-\frac{\partial \bar{H}}{\partial q_{2}}-\mathscr{N}(q) B(q) \frac{\partial \bar{H}}{\partial p_{1}}\right) .
\end{aligned}
$$

Таким образом, справедлив следующий результат.

Теорема 1. Если система (16) допускает инвариантную меру вида (17), то она представляется в конформно-гамильтоновой форме

$$
\dot{q}_{i}=\mathscr{N}(q)\left\{q_{i}, \bar{H}\right\}, \quad \dot{p}_{i}=\mathscr{N}(q)\left\{p_{i}, \bar{H}\right\}, \quad i=1,2,
$$

где скобки Пуассона задаются соотношениями

$$
\left\{q_{i}, p_{j}\right\}=\delta_{i j}, \quad\left\{q_{i}, q_{j}\right\}=0, \quad\left\{p_{1}, p_{2}\right\}=\mathscr{N}(q) B(q) .
$$


ДокАЗАТЕЛЬство заключается в непосредственной проверке тождества Якоби.

Система Чаплыгина на $T^{*} S^{2}$. Пусть система описывается парой трехмерных векторов $M, \gamma$, а уравнения движения имеют вид

$$
\dot{M}=(M-S \gamma) \times \frac{\partial H}{\partial M}+\gamma \times \frac{\partial H}{\partial \gamma}, \quad \dot{\gamma}=\gamma \times \frac{\partial H}{\partial M},
$$

где "гамильтониан" $H(M, \gamma)$ - произвольная функция (квадратичная по $M)$, а $S(M, \gamma)$ - линейная по $M$ функция:

$$
S=(K(\gamma), M)=K_{1}(\gamma) M_{1}+K_{2}(\gamma) M_{2}+K_{3}(\gamma) M_{3}
$$

Непосредственной проверкой доказывается, что система (19) всегда допускает три интеграла движения:

$$
F_{1}=\gamma^{2}=\text { const }, \quad F_{2}=(M, \gamma)=\text { const }, \quad F_{3}=\text { const } .
$$

Без ограничения общности можно полагать $\gamma^{2}=1$, так что уравнения (18) описывают динамическую систему на семействе четырехмерных многообразий

$$
\mathscr{M}_{c}^{4}=\left\{M, \gamma \mid \gamma^{2}=1,(M, \gamma)=c\right\},
$$

каждое из которых диффеоморфно $T S^{2}$.

Если всю совокупность переменных обозначить через $x=(\gamma, M)$, то уравнения (19) можно представить в стандартной кососимметричной форме

$$
\dot{x}=P_{0} \frac{\mathrm{d} H}{\mathrm{~d} x}
$$

относительно бивектора Пуассона

$$
P_{0}=\left(\begin{array}{cc}
0 & \boldsymbol{\Gamma} \\
\Gamma & \mathbf{M}
\end{array}\right)-S(x)\left(\begin{array}{ll}
0 & 0 \\
0 & \boldsymbol{\Gamma}
\end{array}\right)
$$

где

$$
\mathbf{M}=\left(\begin{array}{ccc}
0 & -M_{3} & M_{2} \\
M_{3} & 0 & -M_{1} \\
-M_{2} & M_{1} & 0
\end{array}\right), \quad \boldsymbol{\Gamma}=\left(\begin{array}{ccc}
0 & -\gamma_{3} & \gamma_{2} \\
\gamma_{3} & 0 & -\gamma_{1} \\
-\gamma_{2} & \gamma_{1} & 0
\end{array}\right)
$$

Здесь первое слагаемое - стандартная пуассонова структура, отвечающая алгебре $e(3)$. Более того, $P_{0}$ дополнительно удовлетворяет уравнениям

$$
P_{0} \frac{\partial F_{1}}{\partial x}=0, \quad P_{0} \frac{\partial F_{2}}{\partial x}=0 .
$$

Как и выше, предположим, что система (19) допускает инвариантную меру с плотностью, зависящей лишь от $\gamma$ :

$$
\mu=\rho(\gamma) \mathrm{d} M \mathrm{~d} \gamma
$$

4 УМН, т. 69, вып. 3 
При этом уравнение Лиувилля для векторного поля $V(M, \gamma)$, задаваемого системой (19), представляется в виде

$$
\operatorname{div} \rho V=\left(\frac{\partial H}{\partial M}, \rho \gamma \times K-\gamma \times \frac{\partial \rho}{\partial \gamma}\right)=0 .
$$

Отсюда, вследствие невырожденности гамильтониана по $M$, получим векторное уравнение

$$
\left(\frac{1}{\rho} \frac{\partial \rho}{\partial \gamma}-K\right) \times \gamma=0
$$

используя которое можно доказать следующее предложение.

ПрЕДЛОЖЕНИЕ 1. Если функиия $\rho(\gamma)$ удовлетворяет уравнению (21), то тензор $P=\frac{1}{\rho(\gamma)} P_{0}$ удовлетворяет тождеству Якоби.

Таким образом, мы окончательно получим следующую теорему.

ТЕОрема 2. Если система (19) допускает инвариантную меру (20) с плотностью, зависящей лищь от $\gamma$, то она представляется в конформно-гамильтоновой форме

$$
\dot{x}=\rho(\gamma) P(x) \frac{\partial H}{\partial x},
$$

где $P(x)=\rho^{-1} P_{0}(x)$ - пуассонова структура ранга 4 с функицями Казимира

$$
F_{1}=\gamma^{2}, \quad F_{2}=(M, \gamma)
$$

Уравнение (21) может быть разрешено относительно вектора $K$ следующим образом:

$$
K=\rho f(\gamma) \gamma+\frac{1}{\rho} \frac{\partial \rho}{\partial \gamma},
$$

где $f(\gamma)$ - произвольная функция. Тем самым, естественным образом мы получили специальный класс пуассоновых структур на пространстве $\mathbb{R}^{6}(M, \gamma)$, которые могут быть записаны в следующем виде:

$$
P=\frac{1}{\rho}\left(\begin{array}{cc}
0 & \boldsymbol{\Gamma} \\
\boldsymbol{\Gamma} & \mathbf{M}
\end{array}\right)-\left(\frac{1}{\rho^{2}} \frac{\partial \rho}{\partial \gamma}+f(\gamma)(\gamma, M)\right)\left(\begin{array}{ll}
0 & 0 \\
0 & \boldsymbol{\Gamma}
\end{array}\right) .
$$

Пуассоновы структуры этого типа обсуждаются в работе [84] с точки зрения теории деформаций канонических скобок Пуассона.

ЗАмечаниЕ 5. Добавление к скобке (22) слагаемого вида

$$
\Phi(\gamma)\left(\begin{array}{ll}
0 & 0 \\
0 & \Gamma
\end{array}\right)
$$

где $\Phi(\gamma)$ - произвольная функция, также сохраняет тождество Якоби. 
1.4. Приведение скобок Пуассона к каноническим. Если ввести обозначение $g=\rho^{-1}$, то пуассонову структуру (22) можно переписать в более удобной для исследования форме

$$
P=g\left(\begin{array}{cc}
0 & \boldsymbol{\Gamma} \\
\boldsymbol{\Gamma} & \mathbf{M}
\end{array}\right)+\left(\frac{\partial g}{\partial \gamma}-f \cdot \gamma, M\right)\left(\begin{array}{cc}
0 & 0 \\
0 & \boldsymbol{\Gamma}
\end{array}\right) .
$$

Исследуем класс таких пуассоновых структур более детально. Прежде всего отметим, что параметрами этих пуассоновых структур служат две произвольные функции $g(\gamma)>0$ и $f(\gamma)$. Соответствующую пуассонову структуру мы обозначим поэтому через $P_{g, f}$. Все $P_{g, f}$ обладают одними и теми же функциями Казимира $(M, \gamma)$ и $(\gamma, \gamma)$.

Для простоты мы ограничимся рассмотрением физического случая $\gamma^{2}=$ $(\gamma, \gamma)=1$, т. е. ограничим все объекты на пятимерное (пуассоново) многообразие $S^{2}(\gamma) \times \mathbb{R}^{3}(M)$.

Одна из наших целей - выяснить, к какому каноническому виду приводятся эти пуассоновы структуры. Прежде всего отметим, что симплектические листы $P_{g, f}$ диффеоморфны кокасательному расслоению к сфере $T^{*} S^{2}$. Из явного вида (14) пуассоновой структуры можно сделать вывод, что симплектическая структура на листе $T^{*} S^{2}$ будет суммой канонической формы $\mathrm{d} p \wedge \mathrm{d} q$ и некоторой магнитной добавки, т. е. замкнутой 2-формы $\omega_{\text {magn }}$ на сфере. Согласно теореме Мозера [65], с точностью до симплектоморфизма такие формы $\omega_{\text {magn }}$ параметризуются ровно одним числом, а именно интегралом по сфере $\int_{S^{2}} \omega_{\text {magn }}$. Таким образом, для каждой пуассоновой структуры мы имеем однопараметрическое семейство симплектических листов, тип которых также определяется ровно одним параметром. Это наблюдение приводит к гипотезе о том, что, "перераспределив" при необходимости симплектические листы и применив затем некоторый симплектоморфизм к каждому отдельному симплектическому листу, мы сможем перевести любую структуру $P_{g, f}$ в любую другую $P_{\widetilde{g}}, \tilde{f}$.

ЗАмечание 6 . На нулевом уровне $(M, \gamma)=0$ пуассонова структура (23) приводится к каноническому виду алгебры Ли $e(3)$ простейшей заменой [24]

$$
(M, \gamma) \mapsto\left(g^{-1}(\gamma) M, \gamma\right)
$$

ЗАмЕчАНИЕ 7. Весь этот круг вопросов непосредственно примыкает также к работам С. П. Новикова [70], [71].

Мы начнем с того, что опишем класс естественных преобразований, которые сохраняют вид тензора Пуассона $P_{g, f}$, но меняют параметры $g$ и $f$. Так, рассмотрим преобразования следующего вида:

$$
(M, \gamma) \mapsto(\widetilde{M}, \gamma), \quad \widetilde{M}=\mathbf{A}(\gamma) M,
$$

где $\mathbf{A}(\gamma)$ - некоторый линейный оператор в $\mathbb{R}^{3}$, компоненты которого зависят от $\gamma$. 
ПРЕДЛОЖЕНИЕ 2. Для каждой точки $\gamma \in S^{2}$ рассмотрим ортогональное разложение $M=M^{\prime}+M^{\prime \prime}$, где $M^{\prime \prime}=(M, \gamma) \gamma-$ проекция $M$ на вектор $\gamma$, a $M^{\prime}=M-M^{\prime \prime}$ - проекция $M$ на плоскость, перпендикулярную $\gamma$. Пусть

$$
\widetilde{M}=\alpha(\gamma) M^{\prime}+c M^{\prime \prime}+M^{\prime \prime} \times h(\gamma)
$$

где с - константа, $\alpha(\gamma)>0$ - произвольная скалярная функция, а $h(\gamma)$ - произвольная вектор-функиия от $\gamma$. Тогда преобразование (24) переводит пуассонову структуру $P_{g, f}$ в пуассонову структуру $P_{\widetilde{g}, \tilde{f}}$ аналогичного вида, параметры которой задаются следующим образом:

$$
\widetilde{g}=\alpha g, \quad \tilde{f}=\frac{\alpha^{2}}{c} f+\left(\frac{\alpha}{c}-1\right)\left(\widetilde{g}-\left(\gamma, \frac{\partial \widetilde{g}}{\partial \gamma}\right)\right)+\frac{1}{c}\left(\gamma, \widetilde{g} \frac{\partial \alpha}{\partial \gamma}+\widetilde{g}^{2} \operatorname{rot}\left(\frac{h}{\widetilde{g}}\right)\right) .
$$

ДокАзАТЕльство предложения состоит в непосредственной проверке.

Мы ограничимся комментарием о геометрическом смысле преобразования $M \mapsto \widetilde{M}$, использованном в этом утверждении. Рассмотрим ортонормированный базис $e_{1}, e_{2}, e_{3}$ пространства $\mathbb{R}^{3}(M)$, связанный с вектором $\gamma$. А именно, $e_{1}, e_{2}$ - два ортонормированных вектора, лежащих в касательной плоскости к единичной сфере в точке $\gamma$, а $e_{3}$ - вектор нормали к этой сфере в той же точке, т. е. $e_{3}=\gamma$. В этом базисе матрица оператора $\mathbf{A}$ имеет вид

$$
\mathbf{A}=\left(\begin{array}{lll}
\alpha & 0 & a \\
0 & \alpha & b \\
0 & 0 & c
\end{array}\right)
$$

где $\alpha, a, b$ зависят от $\gamma$, а $c$ - константа.

Это в точности общий вид преобразования $\mathbf{A}$, удовлетворяющего нашим требованиям. Действительно, функция Казимира $(M, \gamma)$ должна перейти в себя с возможным домножением на константу, это и есть $c$. Поэтому плоскость, задаваемая уравнением $(M, \gamma)=0$, должна перейти в себя, а в ортогональном направлении преобразование должно быть растяжением в $c$ раз, причем $c$ не должна зависеть от $\gamma$. Эти условия полностью определяют последнюю строчку матрицы А.

Кроме того, соотношения $\left\{M_{i}, \gamma_{j}\right\}=-g \cdot \varepsilon_{i j k} \gamma_{k}$ можно формально переписать в векторной форме как $\{M, \gamma\}=-g M \times \gamma$. Поскольку их вид должен сохраниться, то мы получаем условие

$$
g(\mathbf{A}(\gamma) M) \times \gamma=\widetilde{g} M \times \gamma,
$$

а это в точности означает, что на касательной плоскости к сфере оператор должен действовать как умножение на некоторое число $\alpha$ (зависящее от $\gamma$ ). На элементы $a, b$ никаких ограничений нет, они задаются вектор-функцией $h$ (сама эта функция имеет три компоненты, но существенными являются только две из них, поскольку добавление к $h$ вектора, пропорционального $\gamma$, ничего не меняет).

Полезно обратить внимание на следующее обстоятельство. Множество преобразований, описанных в предложении 2, образует группу (разумеется, бесконечномерную, поскольку ее параметры содержат произвольные функции $\alpha$ 
и $h)$. Легко проверить, что последовательное выполнение преобразований с параметрами $\left(\alpha_{1}, c_{1}, h_{1}\right)$ и $\left(\alpha_{2}, c_{2}, h_{2}\right)$ эквивалентно выполнению преобразования с параметрами $\left(\alpha_{1} \alpha_{2}, c_{1} c_{2}, h_{1} \alpha_{2}+h_{2} c_{1}\right)$. Указанное правило задает групповой закон, который просто копирует матричное умножение:

$$
\left(\begin{array}{cc}
\alpha_{2} & h_{2} \\
0 & c_{2}
\end{array}\right)\left(\begin{array}{cc}
\alpha_{1} & h_{1} \\
0 & c_{1}
\end{array}\right)=\left(\begin{array}{cc}
\alpha_{1} \alpha_{2} & h_{1} \alpha_{2}+h_{2} c_{1} \\
0 & c_{1} c_{2}
\end{array}\right) .
$$

Эта группа естественным образом действует на рассматриваемом классе пуассоновых структур или, что то же самое, на пространстве параметров $g, f$. Выписанные выше правила (25) - это в точности формулы для этого действия. Если действие формально обозначить через $(\widetilde{g}, \widetilde{f})=\Psi_{(\alpha, c, h)}(g, f)$, то оно, как нетрудно убедиться, выполнив последовательно два преобразования, будет удовлетворять стандартному правилу. А именно, если

$$
(\widetilde{g}, \widetilde{f})=\Psi_{\left(\alpha_{1}, c_{1}, h_{1}\right)}(g, f) \quad \text { и } \quad(\tilde{\tilde{g}}, \tilde{\tilde{f}})=\Psi_{\left(\alpha_{2}, c_{2}, h_{2}\right)}(\widetilde{g}, \widetilde{f}),
$$

TO

$$
(\tilde{\tilde{g}}, \tilde{\tilde{f}})=\Psi_{\left(\alpha_{1} \alpha_{2}, c_{1} c_{2}, h_{1} \alpha_{2}+h_{2} c_{1}\right)}(g, f) .
$$

Для явной проверки этого факта формулы (25) удобно переписать в виде

$$
\tilde{g}=\alpha g, \quad \widetilde{f}=\frac{\alpha^{2}}{c}\left(f+g-\left(\gamma, \frac{\partial g}{\partial \gamma}\right)\right)-\left(\widetilde{g}-\left(\gamma, \frac{\partial \widetilde{g}}{\partial \gamma}\right)\right)+\frac{\widetilde{g}^{2}}{c}\left(\gamma, \operatorname{rot}\left(\frac{h}{\widetilde{g}}\right)\right),
$$

после чего проверка труда не составляет.

C точки зрения теории групп теперь было бы естественно задать вопрос: как устроены орбиты этого действия? Другими словами, мы хотим понять, какие пуассоновы структуры можно перевести друг в друга указанными преобразованиями. Ответ оказывается очень простым: описанное действие имеет единственную орбиту, т. е. все пуассоновы структуры рассматриваемого класса эквивалентны между собой. В частности, справедлива следующая теорема.

Теорема 3. Всякая пуассонова структура $P_{g, f}$ вида (23) на уровне $\gamma^{2}=1$ изоморфна стандартной пуассоновой структуре $P_{1,0}$, отвечающей алгебре $\pi u e(3)$.

ДокАЗАТЕЛЬСтво. Нам достаточно подобрать параметры преобразования $(\alpha, c, h)$ в формулах $(25)$ так, чтобы были выполнены равенства $\widetilde{g}=1$ и $\widetilde{f}=0$. Первое условие сразу определяет функцию $\alpha$, а именно, $\alpha=g^{-1}$. Второе условие после этого существенно упрощается и принимает вид

$$
\frac{\alpha^{2}}{c} f+\left(\frac{\alpha}{c}-1\right)+\frac{1}{c}\left(\gamma, \frac{\partial \alpha}{\partial \gamma}\right)+\frac{1}{c}(\gamma, \operatorname{rot} h)=0
$$

или, эквивалентно,

$$
\alpha^{2} f+\alpha+\left(\gamma, \frac{\partial \alpha}{\partial \gamma}\right)-c+(\gamma, \operatorname{rot} h)=0
$$

где неизвестными являются константа $c$ и вектор-функция $h$. Поэтому уравнение можно теперь переписать в виде

$$
(\gamma, \operatorname{rot} h)=F(\gamma)+c
$$


где $F(\gamma)$ - некоторая заданная нам функция, причем удовлетворить выписанному условию мы должны лишь на единичной сфере $\gamma^{2}=1$. Условия разрешимости уравнения такого вида хорошо известны. В дифференциально-геометрическом смысле это уравнение просто означает, что мы ищем первообразную для 2 -формы вида $(F+c) \mathrm{d} \sigma$ на единичной сфере, где $\mathrm{d} \sigma-$ стандартная форма площади. Это можно сделать тогда и только тогда, когда $\int_{S^{2}}(F+c) \mathrm{d} \sigma=0$, чего всегда можно добиться подбором константы $c$. Теорема доказана.

Замечание 8. Аналогично, возможно приведение скобки $P_{g, f}$ к стандартному виду уже на всей алгебре Ли $e(3)$, т. е. без дополнительного ограничения $\gamma^{2}=1$, для этого класс преобразований придется расширить: необходимо будет предположить, что $c$ зависит от $\gamma^{2}$. Поскольку $\gamma^{2}$ - функция Казимира, то во всех преобразованиях к $c\left(\gamma^{2}\right)$ можно будет относиться по-прежнему как к константе, и поэтому формулы существенно не изменятся. Условия разрешимости уравнения $(\gamma, \operatorname{rot} h)=F(\gamma)+c\left(\gamma^{2}\right)$ остаются прежними, но теперь проверять их нужно на сферах всех радиусов. У нас по-прежнему есть возможность добиться их выполнения, поскольку нужные константы теперь можно выбирать в зависимости от радиуса $\gamma^{2}$.

Так, в задаче о шаре Чаплыгина на плоскости, упоминавшейся выше, в уравнении (26) функция $F(\gamma)$ имеет вид

$$
F(\gamma)=-\frac{\mathscr{D}^{-1}}{\left(\mathscr{D}^{-1}-(\gamma, \mathbf{A} \gamma)\right)^{3 / 2}}
$$

Решения уравнения (26) для неизвестных $c$ и $h$ в этом случае могут быть выражены через полные и неполные эллиптические интегралы. Таким образом, несмотря на то что с теоретической точки зрения доказательство возможности приведения скобки $P_{g, f}$ к $e(3)$-скобке большого труда не составляет, получившееся преобразование может оказаться очень громоздким и неалгебраическим.

Аналогичное преобразование скобки (23) к е(3)-скобке указано в [84], [85], там также отмечено, что если допустить сингулярность на $S^{2}$, решение уравнения (26) может быть выражено в явном виде через элементарные функции.

\section{2. Движение твердого тела по плоскости}

Далее мы будем рассматривать различные случаи качения твердого тела по заданной поверхности под действием внешних сил. Предполагается, что во время движения тело касается поверхности только в одной точке и катится без проскалъзывания. Как известно, данные связи являются неголономными, и соответствующие уравнения движения могут быть получены разнообразными методами (см., например, [2], [3], [23], [30], [50], [68], [69], [75], [94]).

В этом разделе мы приведем только необходимые для нас определения и уравнения для случая качения твердого тела по плоскости. Определим две системы координат (см. рис. 1):

- неподвижная $O X Y Z$ - начало $O$ располагается в некоторой точке плоскости, а ось $O Z$ перпендикулярна плоскости; 


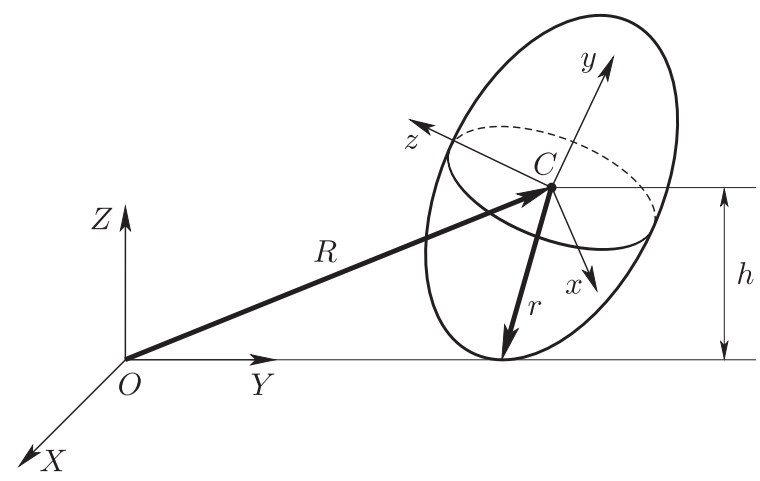

Рис. 1

- подвижная Cxyz - начало $C$ совпадает с центром масс тела, а оси направлены вдоль главных осей инерции.

Пусть $\alpha, \beta, \gamma$ - орты неподвижного пространства (т. е. единичные векторы осей $O X Y Z)$, спроецированные на подвижные оси $C x y z, R=\left(R_{1}, R_{2}, R_{3}\right)$ координаты центра масс тела в неподвижных осях $O X Y Z$; если определить ортогональную матрицу

$$
Q=\left\|\begin{array}{lll}
\alpha_{1} & \beta_{1} & \gamma_{1} \\
\alpha_{2} & \beta_{2} & \gamma_{2} \\
\alpha_{3} & \beta_{3} & \gamma_{3}
\end{array}\right\| \in \operatorname{SO}(3),
$$

то пара $(R, Q) \in \mathbb{R}^{3} \otimes \mathrm{SO}(3)$ однозначно определяет положение тела.

Пусть $v, \omega$ - скорость центра масс и угловая скорость тела, которые будем проецировать на подвижные оси $C x y z$. Вследствие отсутствия проскальзывания в точке контакта ее скорость обращается в нуль:

$$
v+\omega \times r=0,
$$

где $r$ - вектор из центра масс в точку контакта, также спроецированный на подвижные оси.

Если тело всюду выпукло и $\mathfrak{f}(r)=0$ - уравнение его поверхности, то $r$ может быть однозначно выражен через вектор $\gamma$ путем обращения отображения Гаусса

$$
\gamma=-\frac{\nabla \mathfrak{f}(r)}{|\nabla \mathfrak{f}(r)|} .
$$

(Это уравнение выражает тот факт, что нормаль к поверхности тела совпадает с нормалью к плоскости.) В дальнейшем будем полагать, что $r=r(\gamma)-$ заданная функция.

Пользуясь уравнениями динамики твердого тела в подвижных осях Cxyz, после исключения реакции опоры с помощью уравнения связи (28) получим полную систему уравнений, описывающих эволюцию системы:

$$
\begin{gathered}
\mathbf{I} \dot{\omega}+m r \times(\dot{\omega} \times r)+m r \times(\omega \times \dot{r})=\mathbf{I} \omega \times \omega+m(\omega, r) \omega \times r+M_{F}, \\
\dot{\alpha}=\alpha \times \omega, \quad \dot{\beta}=\beta \times \omega, \quad \dot{\gamma}=\gamma \times \omega, \\
\dot{R}_{1}=(\alpha, v), \quad \dot{R}_{2}=(\beta, v), \quad \dot{R}_{3}=(\gamma, v),
\end{gathered}
$$


где $m$ - масса тела, $\mathbf{I}=\operatorname{diag}\left(I_{1}, I_{2}, I_{3}\right)$ - тензор моментов инерции относительно центра масс, $M_{F}$ - момент внешних сил, действующих на тело. В дальнейшем будем полагать, что внешние силы потенциальны с потенциалом $U$, зависящим лишь от $\gamma$, тогда

$$
M_{F}=\gamma \times \frac{\partial U}{\partial \gamma} .
$$

В этом случае система допускает группу симметрий $E(2)$, которая состоит из переносов вдоль плоскости и вращений вокруг вертикали. Вследствие этого в данном случае отделяется шестимерная редуцированная система, описывающая эволюцию векторов $\omega, \gamma$. (Компоненты этих векторов в подвижных осях являются инвариантами действия группы симметрий $E(2)$ на фазовом пространстве системы.) Получившиеся уравнения перепишем в более удобной форме, определив кинетический момент тела относительно точки контакта:

$$
M=\mathbf{I} \omega+m r \times(\omega \times r) .
$$

Разрешая это уравнение относительно $\omega$, получим уравнения на шестимерном приведенном фазовом пространстве $\mathscr{M}$ с координатами $x=(\gamma, M)$ :

$$
\begin{gathered}
\dot{M}=M \times \omega+m \dot{r} \times(\omega \times r)+\gamma \times \frac{\partial U}{\partial \gamma}, \quad \dot{\gamma}=\gamma \times \omega, \\
\omega=\frac{A M+m r \times(A r \times A M)}{1-m(r, A r)}, \quad A=(\mathbf{I}+(r, r))^{-1},
\end{gathered}
$$

которые и будем изучать в дальнейшем.

Векторное поле $X$, отвечающее динамическим уравнениям (32), обладает двумя интегралами

$$
H=\frac{1}{2}(M, \omega)+U(\gamma), \quad C=(\gamma, \gamma)=1
$$

Данная система в зависимости от параметров и возникающих при этом законов сохранения демонстрирует различные типы динамического поведения: от наиболее простого регулярного поведения интегрируемых систем в случае наличия "полного набора законов сохранения" до сложного хаотического поведения, характерного для диссипативных систем, в случае отсутствия инвариантов. Для возможного спектра различных типов поведения неголономных динамических систем при наличии тех или иных тензорных инвариантов в работах [23], [27] предложено название "иерархия динамики".

В рассматриваемых нами ниже специальных случаях векторное поле $X$ обладает дополнительными интегралами и инвариантной мерой.

2.1. Шар Чаплыгина. Рассмотрим качение динамически несимметричного уравновешенного твердого тела со сферической поверхностью радиуса $R$, так называемый шар Чаплыгина [31]. Напомним, что шар называется уравновешенным, если центр масс совпадает с геометрическим центром, и динамически несимметричным, если все три момента инерции отличаются друг от друга. 
В этом случае, согласно (29), получим $r=-R \gamma$ и при отсутствии внешних сил уравнения движения (32) на редуцированном шестимерном фазовом пространстве принимают вид

$$
\dot{M}=M \times \omega, \quad \dot{\gamma}=\gamma \times \omega,
$$

где

$$
M=(\mathbf{I}+d \mathbf{E}) \omega-d(\gamma, \omega) \gamma, \quad d=m b^{2} .
$$

Векторное поле $X$, отвечающее уравнениям движения (34), обладает четырьмя интегралами движения

$$
H_{1}=(M, \omega), \quad H_{2}=(M, M), \quad H_{3}=(\gamma, \gamma), \quad H_{4}=(\gamma, M)
$$

и инвариантной мерой

$$
\mu=g^{-1}(\gamma) \mathrm{d} \gamma \mathrm{d} M, \quad g(\gamma)=\sqrt{1-d(\gamma, \mathbf{A} \gamma)},
$$

где

$$
\mathbf{A}=\left(\begin{array}{ccc}
a_{1} & 0 & 0 \\
0 & a_{2} & 0 \\
0 & 0 & a_{3}
\end{array}\right)=(\mathbf{I}+d \mathbf{E})^{-1}=\left(\begin{array}{ccc}
\left(I_{1}+d\right)^{-1} & 0 & 0 \\
0 & \left(I_{2}+d\right)^{-1} & 0 \\
0 & 0 & \left(I_{3}+d\right)^{-1}
\end{array}\right) .
$$

Таким образом, согласно теореме Эйлера-Якоби, векторное поле $X$ интегрируемо в квадратурах.

2.2. Конформно-гамильтоново представление. Согласно [22], [24], бивектор Пуассона четвертого ранга для шара Чаплыгина

$$
P_{g}=g(\gamma)\left(\begin{array}{cc}
0 & \boldsymbol{\Gamma} \\
\boldsymbol{\Gamma} & \mathbf{M}
\end{array}\right)-\frac{d}{g(\gamma)}(M, \mathbf{A} \gamma)\left(\begin{array}{cc}
0 & 0 \\
0 & \boldsymbol{\Gamma}
\end{array}\right)
$$

состоит из тех же самых $(3 \times 3)$-матриц

$$
\boldsymbol{\Gamma}=\left(\begin{array}{ccc}
0 & \gamma_{3} & -\gamma_{2} \\
-\gamma_{3} & 0 & \gamma_{1} \\
\gamma_{2} & -\gamma_{1} & 0
\end{array}\right), \quad \mathbf{M}=\left(\begin{array}{ccc}
0 & M_{3} & -M_{2} \\
-M_{3} & 0 & M_{1} \\
M_{2} & -M_{1} & 0
\end{array}\right)
$$

что и канонический бивектор Пуассона $P_{0}(10)$, а функция $g \equiv g(\gamma)$ определяет плотность инвариантной меры (37).

Соответствующие скобки Пуассона равны

$$
\left\{M_{i}, M_{j}\right\}_{g}=\varepsilon_{i j k}\left(g M_{k}-\frac{d(M, \mathbf{A} \gamma)}{g} \gamma_{k}\right), \quad\left\{M_{i}, \gamma_{j}\right\}_{g}=\varepsilon_{i j k} g \gamma_{k}, \quad\left\{\gamma_{i}, \gamma_{j}\right\}_{g}=0
$$

где $\varepsilon_{i j k}$ - полностью антисимметрический тензор.

Используя эти скобки Пуассона на полностью редуцированном фазовом пространстве, исходные уравнения движения (34) можно переписать в следующем виде:

$$
\frac{\mathrm{d} x_{k}}{\mathrm{~d} t} \equiv X_{k}=g^{-1}\left\{H, x_{k}\right\}_{g}, \quad \text { где } \quad H=\frac{H_{1}}{2} .
$$

Здесь $H_{1}$ - интеграл движения (36) и, как обычно, $x=\left(\gamma_{1}, \gamma_{2}, \gamma_{3}, M_{1}, M_{2}, M_{3}\right)$. 


\section{3. Приведение скобок Пуассона к каноническим.}

2.3.1. Природа скобок Пуассона (39) может быть объяснена с помощью деформации канонических скобок Пуассона на кокасательных расслоениях и отображения момента (см. также п. 1.4). Действительно, если $g(q)$ - произвольная функция на каком-либо конфигурационном пространстве $Q$ с координатами $q=\left(q_{1}, \ldots, q_{n}\right)$, то, заменив сопряженные переменные $p=\left(p_{1}, \ldots, p_{n}\right)$ в канонической 1-форме Лиувилля и соответствующей симплектической 2-форме

$$
\theta=p_{1} \mathrm{~d} q_{1}+\cdots+p_{n} \mathrm{~d} q_{n}, \quad \Omega=\mathrm{d} \theta,
$$

по правилу

$$
p_{k} \rightarrow g(q) p_{k}
$$

мы получим формы

$$
\theta_{g}=g(q)\left(p_{1} \mathrm{~d} q_{1}+\cdots+p_{n} \mathrm{~d} q_{n}\right), \quad \Omega \rightarrow \Omega_{g}=\mathrm{d} \theta_{g} .
$$

Соответствующие скобки Пуассона

$$
\left\{q_{i}, q_{j}\right\}_{g}=0, \quad\left\{q_{i}, p_{j}\right\}_{g}=g \delta_{i j}, \quad\left\{p_{i}, p_{j}\right\}_{g}=p_{i} \partial_{j} g-p_{j} \partial_{i} g
$$

где $\partial_{k}=\partial / \partial q_{k}$, являются тривиальной деформацией исходных канонических скобок Пуассона

$$
\left\{q_{i}, q_{j}\right\}=0, \quad\left\{q_{i}, p_{j}\right\}=\delta_{i j}, \quad\left\{p_{i}, p_{j}\right\}=0 .
$$

Эти вопросы обсуждаются также в книге [72].

Если теперь отождествить $Q$ с двумерной сферой $S^{2}$, вложенной стандартным образом в $\mathbb{R}^{3}$, так что $q_{i}=\gamma_{i}, i=1,2,3$, то стандартное отображение момента

$$
T^{*} S^{2} \ni(p, \gamma) \rightarrow(M, \gamma) \in\left(\operatorname{so}(3) \ltimes \mathbb{R}^{3}\right)^{*},
$$

определяемое векторным произведением

$$
M=\gamma \times p
$$

отображает скобки Пуассона (42) в скобки Пуассона (23)

$$
\begin{gathered}
\left\{M_{i}, M_{j}\right\}_{g}=\varepsilon_{i j k}\left(g(\gamma) M_{k}+\gamma_{k} \sum_{m=1}^{3} M_{m} \partial_{m} g(\gamma)\right) \\
\left\{M_{i}, \gamma_{j}\right\}_{g}=\varepsilon_{i j k} g(\gamma) \gamma_{k}, \quad\left\{\gamma_{i}, \gamma_{j}\right\}_{g}=0 .
\end{gathered}
$$

Здесь, как и ранее, $\partial_{m}=\partial / \partial \gamma_{m}$.

Если в качестве $g(\gamma)$ взять функцию (37), то скобки Пуассона (44) совпадают со скобками (39), возникающими при рассмотрении неголономного шара Чаплыгина. 
2.3.2. Так как скобки Пуассона (39) являются деформациями канонических скобок Ли-Пуассона специального вида (см. [81]), то они могут быть приведены к этим каноническим скобкам различными способами (см. п. 1.4). Например, в [83] предложена замена переменных, которая при $c \neq 0$ содержит особенность, но представляется через элементарные функции:

$$
\begin{aligned}
& L_{1}=g^{-1}\left(M_{1}-\frac{b \gamma_{1}}{(\gamma, \gamma)}\left(1+\frac{\gamma_{3}^{2}}{\nu}\right)\right)+\frac{c \gamma_{1}}{\gamma_{1}^{2}+\gamma_{2}^{2}}, \\
& L_{2}=g^{-1}\left(M_{2}-\frac{b \gamma_{2}}{(\gamma, \gamma)}\left(1+\frac{\gamma_{3}^{2}}{\nu}\right)\right)+\frac{c \gamma_{2}}{\gamma_{1}^{2}+\gamma_{2}^{2}}, \\
& L_{3}=g^{-1}\left(M_{3}-\frac{b \gamma_{3}}{(\gamma, \gamma)}\left(1-\frac{\gamma_{1}^{2}+\gamma_{2}^{2}}{\nu}\right)\right),
\end{aligned}
$$

где

$$
b=(\gamma, M), \quad c=(\gamma, L), \quad \nu=\gamma_{1}^{2}+\gamma_{2}^{2}-d(\gamma, \gamma)\left(a_{1} \gamma_{1}^{2}+a_{2} \gamma_{2}^{2}\right)
$$

она приводит скобки Пуассона (39) к каноническим скобкам Ли-Пуассона (9) алгебры $e^{*}(3)$. Здесь $b$ и $c$ - числовые значения операторов Казимира, отвечающие связанным друг с другом симплектическим листам.

Применяя обратное к (45) пуассоново отображение

$$
\begin{aligned}
& M_{1}=g\left(L_{1}-\frac{c \gamma_{1}}{\gamma_{1}^{2}+\gamma_{2}^{2}}\right)+\frac{b \gamma_{1}}{(\gamma, \gamma)}\left(1+\frac{\gamma_{3}^{2}}{\nu}\right), \\
& M_{2}=g\left(L_{2}-\frac{c \gamma_{2}}{\gamma_{1}^{2}+\gamma_{2}^{2}}\right)+\frac{b \gamma_{2}}{(\gamma, \gamma)}\left(1+\frac{\gamma_{3}^{2}}{\nu}\right), \\
& M_{3}=g L_{3}+\frac{b \gamma_{3}}{(\gamma, \gamma)}\left(1-\frac{\gamma_{1}^{2}+\gamma_{2}^{2}}{\nu}\right)
\end{aligned}
$$

к исходным интегралам движения (36), мы получим интегрируемую систему на алгебре $e^{*}(3)$ с интегралами движения в инволюции относительно канонических скобок Ли-Пуассона (9).

Более того, если положить $c=0$, то мы получим пуассоново отображение (45) между симплектическими листами скобки Пуассона $\{\cdot, \cdot\}_{g}(39)$ и симплектическим листом скобки Ли-Пуассона $\{\cdot, \cdot\}(9)$

$$
(\gamma, \gamma)=1, \quad(\gamma, L)=0
$$

который симплектоморфен кокасательному расслоению $T^{*} S^{2}$ сферы единичного радиуса. В этом случае исходные интегралы движения (36) имеют вид

$$
\begin{aligned}
\widetilde{H}_{1}= & g^{2}\left(L, \mathbf{A}_{g} L\right)+\frac{2 b g}{\nu}\left((\gamma, \mathbf{A} L)-a_{3} \gamma_{3} L_{3}\right) \\
& +\frac{b^{2}\left(a_{1} \gamma_{1}+a_{2} \gamma_{2}\right)\left(1-d\left(a_{1} \gamma_{1}^{2}+a_{2} \gamma_{2}^{2}\right)\right)}{\nu^{2}}, \\
\widetilde{H}_{2}= & g^{2}(L, L)+\frac{2 b g \gamma_{3} L_{3}}{\nu}+b^{2}\left(1+\frac{\gamma_{3}^{2}\left(\gamma_{1}^{2}+\gamma_{2}^{2}\right)}{\nu^{2}}\right) .
\end{aligned}
$$


ЗАмечАниЕ 9. Используя затем сферо-конические координаты на сфеpe $u_{1}, u_{2}$, мы получим следующие выражения для интегралов движения на $T^{*} S^{2}$ :

$$
\begin{aligned}
& \widetilde{H}_{1}=\mathrm{a} p_{u_{1}}^{2}+2 \mathrm{~b} p_{u_{1}} p_{u_{2}}+\mathrm{c} p_{u_{2}}^{2}+\mathrm{d} p_{u_{1}}+\mathrm{e} p_{u_{2}}+\mathrm{f}, \\
& \widetilde{H}_{2}=\mathrm{A} p_{u_{1}}^{2}+2 \mathrm{~B} p_{u_{1}} p_{u_{2}}+\mathrm{C} p_{u_{2}}^{2}+\mathrm{D} p_{u_{1}}+\mathrm{E} p_{u_{2}}+\mathrm{F} .
\end{aligned}
$$

Здесь $p_{u_{1,2}}-$ моменты, сопряженные координатам $u_{1,2}$, а коэффициенты полиномов в (48) являются аналитическими функциями от $u_{1,2}$. Согласно [61], гамильтоновы системы с такими интегралами допускают частичное разделение переменных в уравнении Гамильтона-Якоби. Переменные разделения $s_{1,2}$ в этом случае являются корнями уравнения

$$
(\mathrm{B}-\mathrm{b} s)^{2}-(\mathrm{A}-\mathrm{a} s)(\mathrm{C}-\mathrm{c} s)=0,
$$

которые в нашем случае равны

$$
s_{1,2}=d\left(1+u_{1,2}\right) u_{1,2}^{-1} .
$$

Как и для случая Клебша [61], эти переменные эволюционируют на примиане алгебраической кривой, которая не является гиперэллиптической, в отличие от разделения переменных Чаплыгина [20], [31].

2.4. Гиростатические обобщения. Следуя [24], рассмотрим, как меняется пуассонова структура (38) при добавлении к телу равномерно вращающегося уравновешенного ротора. Соответствующую систему можно интерпретировать как неголономный гиростат. Эффект гиростата также может быть достигнут при включении в тело многосвязных полостей, полностью заполненных идеальной несжимаемой жидкостью.

В этом случае уравнения движения (32) изменятся следующим образом:

$$
\dot{M}=(M+S) \times \omega+m \dot{r} \times(\omega \times r)+M_{F}, \quad \dot{\gamma}=\gamma \times \omega,
$$

где $S$ - постоянный трехмерный вектор гиростатического момента. Для шара Чаплыгина на плоскости этот вектор произволен, а интегралы движения (36) меняются следующим образом:

$$
H_{1}=(M, \omega), \quad H_{2}=(M+S, M+S), \quad H_{3}=(\gamma, \gamma), \quad H_{4}=(\gamma, M+S) .
$$

Соответствующий уравнениям (49) и новым интегралам движения бивектор Пуассона имеет вид

$$
P_{s g}=g\left(\begin{array}{cc}
0 & \boldsymbol{\Gamma} \\
\boldsymbol{\Gamma} & \mathbf{M}+\mathbf{S}
\end{array}\right)-d g^{-1}(M, \mathbf{A} \gamma)\left(\begin{array}{cc}
0 & 0 \\
0 & \boldsymbol{\Gamma}
\end{array}\right)
$$

где

$$
\mathbf{S}=\left(\begin{array}{ccc}
0 & S_{3} & -S_{2} \\
-S_{3} & 0 & S_{1} \\
S_{2} & -S_{1} & 0
\end{array}\right)
$$

В этом случае векторное поле по-прежнему остается конформно-гамильтоновым:

$$
X=g^{-1} P_{s g} \mathrm{~d} H .
$$

Соответствующие скобки Пуассона были найдены в работе [24]. 


\section{3. Шар Чаплыгина на сфере - БМФ-система}

Следуя [16], [24], рассмотрим динамически несимметричное уравновешенное твердое тело со сферической поверхностью радиуса $b$, которое катится без проскальзывания по поверхности неподвижной сферы радиуса $a$. В этом случае группа симметрий $\mathrm{SO}(3)$ состоит из вращений неподвижного основания, по которому происходит качение.

В подвижной системе координат, связанной с главными осями двигающегося тела, уравнения движения редуцированной системы зависят от отношения радиусов сфер $\kappa=a /(a+b)$ :

$$
\dot{M}=M \times \omega, \quad \dot{\gamma}=\kappa \gamma \times \omega,
$$

где $M$ - угловой момент относительно точки контакта - по-прежнему задается соотношением (35). При любом $\kappa$ уравнения движения (50) обладают тремя интегралами движения

$$
H_{1}=(M, \omega), \quad H_{2}=(M, M), \quad H_{3}=(\gamma, \gamma)
$$

и инвариантной мерой

$$
\mu=g^{-1}(\gamma) \mathrm{d} \gamma \mathrm{d} M, \quad g(\gamma)=\sqrt{1-d(\gamma, \mathbf{A} \gamma)},
$$

где

$$
\mathbf{A}=\left(\begin{array}{ccc}
a_{1} & 0 & 0 \\
0 & a_{2} & 0 \\
0 & 0 & a_{3}
\end{array}\right)=(\mathbf{I}+d \mathbf{E})^{-1}=\left(\begin{array}{ccc}
\left(I_{1}+d\right)^{-1} & 0 & 0 \\
0 & \left(I_{2}+d\right)^{-1} & 0 \\
0 & 0 & \left(I_{3}+d\right)^{-1}
\end{array}\right)
$$

Если $\kappa= \pm 1$, то существует еще один интеграл движения

$$
H_{4}=(\gamma, \mathbf{B} M), \quad \mathbf{B}=\left(\begin{array}{ccc}
b_{1} & 0 & 0 \\
0 & b_{2} & 0 \\
0 & 0 & b_{3}
\end{array}\right)=\operatorname{tr} \mathbf{A}^{-1}+(\kappa-1) \mathbf{A}^{-1}
$$

Значение $\kappa=1$ отвечает предельному случаю, когда опорная поверхность становится плоской, т. е. получается рассматриваемая ранее задача о шаре Чаплыгина (см. п. 2.4).

При $\kappa=-1$ интегрируемость уравнений движения (50) была доказана в работе [16], а конформно-гамильтоново представление и явные квадратуры при $H_{4}=0$ получены в работе [17], поэтому для краткости, следуя [82], эту систему мы будем называть БМФ-системой или системой Борисова-Мамаева-Федорова.

\section{1. Скобки Пуассона и конформно-гамильтоново представление.} При $\kappa=-1$ возникает более сложный бивектор Пуассона, имеющий тем не менее сходную структуру с бивектором $P_{g}(38)$. Действительно, легко проверить, что интегралы движения (51), (53) находятся в инволюции относительно скобок Пуассона, задаваемых линейным по $M$ бивектором Пуассона:

$$
P_{b}=g\left(\begin{array}{cc}
0 & \widehat{\boldsymbol{\Gamma}} \\
-\widehat{\boldsymbol{\Gamma}}^{\top} & \widehat{\mathbf{M}}
\end{array}\right)+g^{-1}(2 d(\gamma, \gamma)-\operatorname{tr} \mathbf{B})\left(\begin{array}{cc}
0 & 0 \\
0 & \widetilde{\boldsymbol{\Gamma}}
\end{array}\right),
$$


причем выполнено равенство $\left[P_{b}, P_{b}\right]=0$. Матрица $\widehat{\boldsymbol{\Gamma}}$ зависит только от $\gamma$ :

$$
\widehat{\boldsymbol{\Gamma}}=\left((\gamma, \gamma) \mathbf{E}-\mathbf{C}-\frac{\mathbf{B}}{2 d}\right) \boldsymbol{\Gamma}_{b}
$$

где $\mathbf{E}$ - единичная матрица,

$$
\mathbf{C}=\left(\begin{array}{ccc}
\gamma_{1}^{2} & \gamma_{1} \gamma_{2} & \gamma_{1} \gamma_{3} \\
\gamma_{2} \gamma_{1} & \gamma_{2}^{2} & \gamma_{2} \gamma_{3} \\
\gamma_{3} \gamma_{1} & \gamma_{3} \gamma_{2} & \gamma_{3}^{2}
\end{array}\right), \quad \boldsymbol{\Gamma}_{b}=\left(\begin{array}{ccc}
0 & b_{3} \gamma_{3} & -b_{2} \gamma_{2} \\
-b_{3} \gamma_{3} & 0 & b_{1} \gamma_{1} \\
b_{2} \gamma_{2} & -b_{1} \gamma_{1} & 0
\end{array}\right)
$$

а элементы оставшихся двух матриц $\widehat{\mathbf{M}}$ и $\widetilde{\boldsymbol{\Gamma}}$ имеют вид

$$
\begin{aligned}
& \widehat{M}_{i j}=-\varepsilon_{i j k}\left(\alpha_{k} \gamma_{k}-(\gamma, \gamma) b_{k} M_{k}+\frac{b_{k}^{2} M_{k}}{2 d}\right), \\
& \widetilde{\Gamma}_{i j}=-\frac{\varepsilon_{i j k} b_{k} \gamma_{k}}{\left(b_{1}+b_{2}\right)\left(b_{2}+b_{3}\right)\left(b_{1}+b_{3}\right)}\left(\left(b_{i}+b_{j}\right) \alpha_{k}+\left(b_{k}-b_{i}\right)\left(b_{k}-b_{j}\right) M_{k} \gamma_{k}\right),
\end{aligned}
$$

где

$$
\alpha_{k}=C_{2}+b_{k}(\gamma, M), \quad C_{2}=(\gamma, \mathbf{B} M) .
$$

Данный бивектор является единственным решением уравнений (12) в классе линейных по $M$ бивекторов Пуассона. Доказательство состоит в непосредственном решении уравнений (12) с использованием линейного по $M_{i}$ анзаца для бивектора $P$ наиболее общего вида (13).

Используя данную пуассонову структуру, исходные уравнения движения (50) можно переписать в форме разложения по конформно-гамильтоновым полям:

$$
\frac{\mathrm{d} x_{k}}{\mathrm{~d} t} \equiv X_{k}=g_{1}^{-1}\left\{H_{1}, x_{k}\right\}_{b}+g_{2}^{-1}\left\{H_{2}, x_{k}\right\}_{b},
$$

где

и

$$
g_{1}(\gamma)=\frac{g(\gamma) s(\gamma)}{(2 d(\gamma, \gamma)-\operatorname{tr} \mathbf{B}) d}, \quad g_{2}(\gamma)=\frac{g(\gamma) s(\gamma)}{2 d}
$$

$$
s(\gamma)=4 d^{2}(\gamma, \gamma)(\gamma, \mathbf{B} \gamma)-2 d((\mathbf{E} \operatorname{tr} \mathbf{B}-\mathbf{B}) \gamma, \mathbf{B} \gamma)+\operatorname{det} \mathbf{B} .
$$

Таким образом, в отличие от обычного шара Чаплыгина, исходное векторное поле не является конформно-гамильтоновым относительно линейной по $M$ пуассоновой структуры, если в качестве гамильтониана выбирается интеграл энергии $H_{1}$. Тем не менее, если поменять гамильтониан, то можно получить представление (17).

ПРЕДЛОЖЕНИЕ 3. Векторное поле X системы (50) при $\kappa=-1$ является конформно-гамильтоновым относительно пуассоновой структуры (54):

$$
X=\left(\frac{g(\gamma) s(\gamma)}{d}\right)^{-1} P_{b} \mathrm{~d} H
$$

с гамильтонианом

$$
H=(2 d(\gamma, \gamma)-\operatorname{tr} \mathbf{B}) H_{1}+2 H_{2}
$$

ДоКАЗАТЕЛЬСтво состоит в непосредственном построении уравнений движения с помощью тензора Пуассона $P_{b}$ и интегралов движения $H_{1,2}$ и $H_{3}$. 
3.2. Приведение скобок Пуассона к каноническим. Как и для шара Чаплыгина на плоскости, в данном случае также существует замена переменных

$$
\begin{aligned}
& L_{1}= \frac{1}{\mathrm{~g}(\gamma) s(\gamma) b_{1} b_{2}\left(\gamma_{1}^{2}+\gamma_{2}^{2}\right)}\left(\alpha_{1}\left(b_{1} \gamma_{2} M_{1}-b_{2} \gamma_{1} M_{2}\right)+\beta_{1} M_{3}+\frac{b \gamma_{1} \gamma_{3} h(\gamma)}{\gamma_{1}^{2}+\gamma_{2}^{2}}\right) \\
& \quad+\frac{c \gamma_{1}}{\gamma_{1}^{2}+\gamma_{2}^{2}}, \\
& L_{2}= \frac{1}{\mathrm{~g}(\gamma) s(\gamma) b_{1} b_{2}\left(\gamma_{1}^{2}+\gamma_{2}^{2}\right)}\left(\alpha_{2}\left(b_{1} \gamma_{2} M_{1}-b_{2} \gamma_{1} M_{2}\right)+\beta_{2} M_{3}+\frac{b \gamma_{2} \gamma_{3} h(\gamma)}{\gamma_{1}^{2}+\gamma_{2}^{2}}\right) \\
& \quad+\frac{c \gamma_{2}}{\gamma_{1}^{2}+\gamma_{2}^{2}}, \\
& L_{3}= \frac{1}{\mathrm{~g}(\gamma) s(\gamma) b_{1} b_{2}\left(\gamma_{1}^{2}+\gamma_{2}^{2}\right)}\left(\alpha_{3}\left(b_{1} \gamma_{2} M_{1}-b_{2} \gamma_{1} M_{2}\right)+\beta_{3} M_{3}-b h(\gamma)\right), \\
& \quad b=(\mathbf{B} \gamma, M), \quad c=(\gamma, L),
\end{aligned}
$$

приводящая $P_{b}$ к каноническому бивектору $P_{0}(10)$. Здесь функция $s(\gamma)$ определена соотношением (56), а

$$
\begin{aligned}
& \alpha_{1}=2 d \gamma_{2}\left(2 d b_{1} \gamma_{1}^{2}+2 d b_{2}\left(\gamma_{2}^{2}+\gamma_{3}^{2}\right)-b_{1} b_{2}\right), \\
& \alpha_{2}=-2 d \gamma_{1}\left(2 d b_{1}\left(\gamma_{1}^{2}+\gamma_{3}^{2}\right)+2 d b_{2} \gamma_{2}^{2}-b_{1} b_{2}\right), \\
& \alpha_{3}=4 d^{2} \gamma_{1} \gamma_{2} \gamma_{3}\left(b_{1}-b_{2}\right),
\end{aligned}
$$

и

$$
\begin{aligned}
& \beta_{1}=-2 d \gamma_{1} \gamma_{3}\left(2 d\left(\gamma_{1}^{2}\left(b_{1} b_{2}-b_{1} b_{3}+b_{2} b_{3}\right)+b_{2}\left(b_{1} \gamma_{1}^{2}+b_{3} \gamma_{3}^{2}\right)\right)-b_{1} b_{2} b_{3}\right), \\
& \beta_{2}=-2 d \gamma_{2} \gamma_{3}\left(2 d\left(\gamma_{1}^{2}\left(b_{1} b_{3}+b_{1} b_{2}-b_{2} b_{3}\right)+b_{1}\left(b_{2} \gamma_{2}^{2}+b_{3} \gamma_{3}^{2}\right)\right)-b_{1} b_{2} b_{3}\right), \\
& \beta_{3}=4 d^{2} b_{3} \gamma_{3}^{2}\left(b_{2} \gamma_{1}^{2}+b_{1} \gamma_{2}^{2}\right)+2 d b_{1} b_{2}\left(\gamma_{1}^{2}+\gamma_{2}^{2}\right)\left(2 d\left(\gamma_{1}^{2}+\gamma_{2}^{2}\right)-b_{3}\right) .
\end{aligned}
$$

Мы не приводим здесь явное выражение для функции $h(\gamma)$, которая является весьма громоздким решением дифференциальных уравнений $\left\{L_{i}, L_{j}\right\}_{b}=$ $\varepsilon_{i j k} L_{k}$. Заметим только, что такое решение существует и не является единственным. (Эта замена также содержит особенность при $c \neq 0$.)

Используя эту замену переменных при $c=(\gamma, L)=0$, мы, как и для шара Чаплыгина, получим динамическую систему на кокасательном расслоении двумерной сферы $T^{*} S^{2}$ с каноническими скобками Пуассона.

\section{4. Система Веселовой}

Рассмотрим движение твердого тела вокруг неподвижной точки в отсутствие внешних сил при наличии неголономной связи

$$
(\omega, \gamma)=0
$$

В данном случае проекция угловой скорости на вектор $\gamma$ равна нулю, что означает отсутствие верчения вокруг вектора $\gamma$ [92], [93]. Уравнения движения имеют вид

$$
\dot{M}=M \times \omega+\lambda \gamma, \quad \dot{\gamma}=\gamma \times \omega
$$


Здесь $M$ - момент количества движения, $\omega=\widehat{\mathbf{A}} M-$ угловая скорость тела, $\gamma-$ орт неподвижной оси в подвижной системе координат и $\widehat{\mathbf{A}}=\mathbf{I}^{-1}$ - диагональная невырожденная матрица (обратная тензору инерции):

$$
\widehat{\mathbf{A}}=\left(\begin{array}{ccc}
\widehat{a}_{1} & 0 & 0 \\
0 & \widehat{a}_{2} & 0 \\
0 & 0 & \widehat{a}_{3}
\end{array}\right)
$$

Дифференцируя в силу системы (58) уравнение связи (57), находим множитель Лагранжа

$$
\lambda=\frac{(\widehat{\mathbf{A}} M \times M, \widehat{\mathbf{A}} \gamma)}{(\widehat{\mathbf{A}} \gamma, \gamma)} .
$$

Векторное поле $X$ имеет четыре интеграла (на уровне связи)

$$
\widehat{H}_{1}=(M, \widehat{\mathbf{A}} M), \quad \widehat{H}_{2}=(M, M)-(\gamma, \gamma)^{-1}(\gamma, M)^{2}, \quad \widehat{H}_{3}=(\gamma, \gamma), \quad \widehat{H}_{4}=(\gamma, \omega)
$$

и инвариантную меру

$$
\mu=\widehat{g} \mathrm{~d} \gamma \mathrm{d} M, \quad \widehat{g}(\gamma)=(\gamma, \widehat{\mathbf{A}} \gamma)^{1 / 2}
$$

Как показано в [24], эта система эквивалентна задаче о качении шара Чаплыгина по плоскости при отсутствии верчения.

Интегралы движения находятся в инволюции относительно скобок Пуассона

$$
\begin{aligned}
\left\{M_{i}, M_{j}\right\}_{a g} & =\frac{\varepsilon_{i j k}}{a_{i} a_{j}}\left(a_{k} \widehat{g}^{-1} M_{k}+\gamma_{k} \sum_{m=1}^{3} a_{m} M_{m} \partial_{m} \widehat{g}^{-1}\right), \\
\left\{M_{i}, \gamma_{j}\right\}_{a g} & =\varepsilon_{i j k} \widehat{g}^{-1} a_{i}^{-1} \gamma_{k}, \quad\left\{\gamma_{i}, \gamma_{j}\right\}_{a g}=0
\end{aligned}
$$

при выполнении условия (57).

4.1. Эквивалентность системы Веселовой и шара Чаплыгина. Можно проверить, что система (58) остается интегрируемой и в случае неоднородных связей [38] (см. также [13], [83]), т. е. вместо (57) получим

$$
(\omega, \gamma)=b, \quad b \in \mathbb{R} .
$$

Вместо $M$ удобно ввести постоянный в пространстве вектор $K$ по правилу

$$
K=M-\frac{((\mathbf{E}-\widehat{\mathbf{A}}) M, \gamma)}{(\gamma, \gamma)}, \quad(\gamma, K)=(\gamma, \omega)=b,
$$

при этом уравнения движения (58) для системы Веселовой примут вид

$$
\dot{K}=K \times \omega, \quad \dot{\gamma}=\gamma \times \omega
$$

где $\omega=\widehat{\mathbf{A}} M$.

Так как якобиан преобразования координат пропорционален квадрату плотности инвариантной меры в исходной задаче Веселовой

$$
\left|\frac{\partial(\gamma, M)}{\partial(\gamma, K)}\right|=\widehat{g}^{2}(\gamma)=(\gamma, \widehat{\mathbf{A}} \gamma)
$$


то данное преобразование координат обратимо (там, где существует инвариантная мера) и, следовательно, корректно определена замена времени, позволяющая проинтегрировать уравнения движения [93].

В новых переменных инвариантная мера имеет вид

$$
\mu=\widehat{g}^{-1} \mathrm{~d} \gamma \mathrm{d} K, \quad \widehat{g}(\gamma)=(\gamma, \widehat{\mathbf{A}} \gamma)^{1 / 2},
$$

а интегралы движения равны

$$
\widehat{H}_{1}=\left(K, \widehat{\mathbf{A}}_{g} K\right), \quad \widehat{H}_{2}=(K, K), \quad \widehat{H}_{3}=(\gamma, \gamma), \quad \widehat{H}_{4}=(\gamma, K),
$$

где

$$
\widehat{\mathbf{A}}_{g}=\widehat{\mathbf{A}}-\widehat{g}^{-2}(\mathbf{E}-\widehat{\mathbf{A}})(\gamma \otimes \gamma)(\mathbf{E}-\widehat{\mathbf{A}}) .
$$

Данные интегралы движения находятся в инволюции относительно скобок Пуассона

$$
\begin{aligned}
\left\{K_{i}, K_{j}\right\}_{v} & =\varepsilon_{i j k} \widehat{g} K_{k}+\varepsilon_{i j k} \gamma_{k}\left(\sum_{m=1}^{3} K_{m} \partial_{m} \widehat{g}-\frac{b}{\widehat{g}}\right), \\
\left\{K_{i}, \gamma_{j}\right\}_{v} & =\varepsilon_{i j k} \widehat{g} \gamma_{k}, \quad\left\{\gamma_{i}, \gamma_{j}\right\}_{v}=0,
\end{aligned}
$$

для которых $(\gamma, \gamma)$ и $(\gamma, K)$ являются функциями Казимира.

Пользуясь этой пуассоновой структурой, уравнения движения (62) можно переписать в конформно-гамильтоновой форме

$$
\frac{\mathrm{d}}{\mathrm{d} t} x_{i}=\widehat{g}^{-1}(\gamma)\left\{H, x_{i}\right\}_{v}, \quad H=\frac{\widehat{H}_{1}}{2}, \quad i=1, \ldots, 6 .
$$

ПРЕДЛОЖЕНИЕ 4. СКобки Пуассона $\{\cdot, \cdot\}_{v}$ (65) и инвариантная мера (63) для системы Веселовой совпадают со скобками Пуассона $\{\cdot, \cdot\}_{g}(39)$ и инвариантной мерой (37) для шара Чапльгина, если $(\gamma, \gamma)=1$ и параметры задач связаны соотношением

$$
\widehat{a}_{i}=1-d a_{i} .
$$

Интегралы движения $\widehat{H}_{i}(64)$ системы Веселовой выражаются через интегралы движения $H_{i}(51)$ системы Чаплыгина:

$$
\widehat{H}_{1}=H_{2}-d H_{1}, \quad \widehat{H}_{2}=H_{2}, \quad \widehat{H}_{3}=H_{3}, \quad \widehat{H}_{4}=H_{4} .
$$

Поскольку наборы интегралов данных систем совпадают (после соответствующей замены параметров), они определяют одно и то же слоение фазового пространства на инвариантные торы.

ЗАмЕчАниЕ 10 . Если $(\gamma, \gamma) \neq 1$, то связь (67) между параметрами задач имеет вид $\widehat{a}_{i}=(\gamma, \gamma)^{-1}-d a_{i}$ и $\widehat{H}_{1}=(\gamma, \gamma)^{-1} H_{2}-d H_{1}$.

Как следствие, скобки Пуассона $\{\cdot, \cdot\}_{v}$ для системы Веселовой приводятся к каноническим скобкам Пуассона на алгебре $e^{*}(3)$ преобразованием

$$
\begin{aligned}
& L_{1}=\widehat{g}^{-1}\left(K_{1}-\frac{b \gamma_{1}}{(\gamma, \gamma)}\left(1+\frac{\gamma_{3}^{2}}{\nu}\right)\right)+\frac{c \gamma_{1}}{\gamma_{1}^{2}+\gamma_{2}^{2}}, \\
& L_{2}=\widehat{g}^{-1}\left(K_{2}-\frac{b \gamma_{2}}{(\gamma, \gamma)}\left(1+\frac{\gamma_{3}^{2}}{\nu}\right)\right)+\frac{c \gamma_{2}}{\gamma_{1}^{2}+\gamma_{2}^{2}}, \\
& L_{3}=\widehat{g}^{-1}\left(K_{3}-\frac{b \gamma_{3}}{(\gamma, \gamma)}\left(1-\frac{\gamma_{1}^{2}+\gamma_{2}^{2}}{\nu}\right)\right), \quad \nu=\widehat{a}_{1} \gamma_{1}^{2}+\widehat{a}_{2} \gamma_{2}^{2},
\end{aligned}
$$


совпадающим с преобразованием (45) с точностью до замены параметров (67).

В работе [13] показано, что эта аналогия между конформно-гамильтоновыми представлениями (на уровне пуассоновых структур) системы Веселовой и задачей о шаре Чаплыгина сохранится при добавлении потенциального поля внешних сил с потенциалом $U(\gamma)$. При этом обе системы в общем случае оказываются неинтегрируемыми.

4.2. Гиростатическое обобщение. Рассмотрим теперь ситуацию, когда к твердому телу добавляется ротор, обладающий гироскопическим моментом $k$. Тогда непосредственным вычислением можно показать, что система остается конформно-гамильтоновой:

$$
\dot{x}=g^{-1} \mathbf{P}_{k}(x) \frac{\partial H}{\partial x},
$$

относительно бивектора Пуассона $\mathbf{P}_{k}$ более общего вида

$$
\mathbf{P}_{k}(x)=g\left(\begin{array}{cc}
0 & \boldsymbol{\Gamma} \\
\boldsymbol{\Gamma} & \mathbf{K}_{k}
\end{array}\right)-g S\left(\begin{array}{cc}
0 & 0 \\
0 & \boldsymbol{\Gamma}
\end{array}\right),
$$

где

$$
\begin{gathered}
\mathbf{K}_{k}=\left(\begin{array}{ccc}
0 & -K_{3}-k_{3} & K_{2}+k_{2} \\
K_{3}+k_{3} & 0 & -K_{1}-k_{1} \\
-K_{2}-k_{2} & K_{1}+k_{1} & 0
\end{array}\right), \\
S=\left(-\frac{\partial g}{\partial \gamma}+f(\gamma) \gamma, K\right)+\Phi(\gamma) .
\end{gathered}
$$

Здесь $x=(\gamma, K)$ - полный набор переменных, а $g$ - функция только от $\gamma$. Тождество Якоби при этом заведомо выполнено, а функции Казимира принимают вид

$$
F_{1}=\gamma^{2}, \quad F_{2}=(K+k, \gamma) .
$$

При добавлении гиростата соотношения (61) необходимо модифицировать. Будем полагать, что $K=\widehat{\mathbf{A}}^{-1} \omega+\lambda \gamma$, где коэффициент $\lambda$ найдем из условия

$$
(K+k, \gamma)=(\omega, \gamma)
$$

Получим

$$
\begin{gathered}
K=\widehat{\mathbf{A}}^{-1} \omega-\left(\left(\widehat{\mathbf{A}}^{-1}-\mathbf{E}\right) \omega+k, \gamma\right) \gamma, \quad \omega=\widehat{\mathbf{A}}(K-S \gamma), \\
S=\frac{(\widehat{\mathbf{A}} K-K-k, \gamma)}{(\widehat{\mathbf{A}} \gamma, \gamma)},
\end{gathered}
$$

здесь $S$ совпадает с соответствующей функцией в скобке $(70)$ при условии, что $g$ задается формулой

$$
g=\sqrt{(\widehat{\mathbf{A}} \gamma, \gamma)}
$$

При этом гамильтониан имеет вид

$$
H=\frac{1}{2}\left((\widehat{\mathbf{A}} K, K)+\frac{(\widehat{\mathbf{A}} K-K-k, \gamma)^{2}}{(\widehat{\mathbf{A}} \gamma, \gamma)}\right)
$$




\section{5. Тело вращения на плоскости}

Начиная с работ [3], [30], [42], [50], [75] исследованию качения без проскальзывания тела вращения по плоскости посвящено большое количество работ см., например, библиографию в [23], [33]. Здесь мы в основном будем следовать работе [23].

Напомним, что для тела вращения его поверхность и центральный эллипсоид инерции являются двумя соосными поверхностями вращения. С математической точки зрения это означает, что мы накладываем следующие ограничения:

$$
I_{1}=I_{2} \neq I_{3}, \quad r_{1}=f_{1}\left(\gamma_{3}\right) \gamma_{1}, \quad r_{2}=f_{1}\left(\gamma_{3}\right) \gamma_{2}, \quad r_{3}=f_{2}\left(\gamma_{3}\right)
$$

Поскольку форма поверхности задается при помощи одного уравнения $\mathfrak{f}(r)=0$, функции $f_{1,2}\left(\gamma_{3}\right)$ в $(71)$ удовлетворяют уравнению

$$
\frac{\mathrm{d} f_{2}\left(\gamma_{3}\right)}{\mathrm{d} \gamma_{3}}=f_{1}\left(\gamma_{3}\right)-\frac{1-\gamma_{3}^{2}}{\gamma_{3}} \frac{\mathrm{d} f_{1}\left(\gamma_{3}\right)}{\mathrm{d} \gamma_{3}}
$$

определяющему меридианальное сечение поверхности вращения. С физической точки зрения это просто следствие равенства нулю проекции скорости изменения вектора $r$ на вектор нормали: $(\dot{r}, \gamma)=0$.

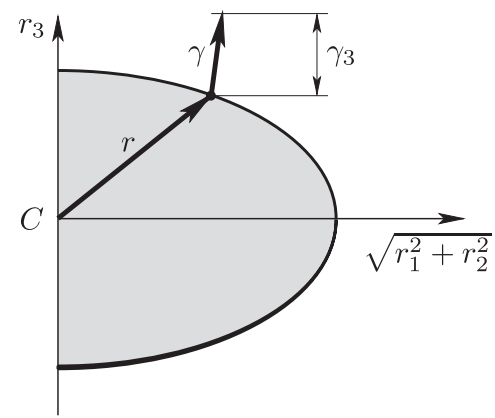

Рис. 2. Меридианальное сечение тела вращения

Кроме этого, предполагается, что потенциал внешних сил $U$ зависит только от $\gamma_{3}$, т. е. только от угла между осью вращения и вертикалью. Для движения в поле тяжести это ограничение означает, что центр масс тела расположен на оси вращения.

В данном случае для общей системы (30) группа симметрий $G=E(2) \times \mathrm{SO}(2)$ состоит из двух подгрупп. Подгруппа движений плоскости $E(2)$ отмечалась выше (см. раздел 1); вторая подгруппа $\mathrm{SO}(2)$ состоит из вращений тела вокруг оси вращения $C z$ (рис. 2), которые иногда называют внутренними симметриями тела [35], [36]. 
Таким образом, векторное поле $X$ приведенной системы (32) инвариантно относительно действия группы $\mathrm{SO}(2)$

$$
\left(\begin{array}{c}
\gamma_{1} \\
\gamma_{2} \\
\gamma_{3} \\
M_{1} \\
M_{2} \\
M_{3}
\end{array}\right) \rightarrow\left(\begin{array}{c}
\cos \theta \gamma_{1}-\sin \theta \gamma_{2} \\
\sin \theta \gamma_{1}+\cos \theta \gamma_{2} \\
\gamma_{3} \\
\cos \theta M_{1}-\sin \theta M_{2} \\
\sin \theta M_{1}+\cos \theta M_{2} \\
M_{3}
\end{array}\right)
$$

которое не является свободным из-за наличия двух стационарных точек

$$
e_{ \pm}=\left(\begin{array}{c}
0 \\
0 \\
\pm 1 \\
0 \\
0 \\
M_{3}
\end{array}\right)
$$

Эти точки соответствуют двум семействам относительных равновесий (параметризуемых величиной $M_{3}$ ), для которых тело вращается вокруг собственной оси, расположенной вертикально.

ЗАмечАние 11. В некоторых работах (см., например, [48]) используется терминология, согласно которой если действие группы симметрий системы обладает неподвижными точками, то она не является системой Чаплыгина.

ЗАмечание 12. Точки (73) могут оказаться на одном и том же интегральном многообразии. В этой ситуации в работе [34] высказана гипотеза, что могут существовать препятствия к гамильтонизации неголономной системы негамильтонова монодромия. Причем в качестве конкретного примера такой системы авторы [34] указывают задачу о качении вытянутого уравновешенного эллипсоида вращения. Как показано в работе [14], в указанной задаче негамильтоновой монодромии не возникает, так как ее лиувиллево слоение эквивалентно слоению гамильтоновой системы, описывающей качение эллипсоида по абсолютно гладкой плоскости. Кроме того, в [14] показано, как монодромия может быть использована при гамильтонизации системы для подходящего выбора функций Казимира.

Генератор действия (72) группы $\mathrm{SO}(2)$, или поле симметрий $X_{S}$, имеет вид

$$
X_{S}=\gamma_{1} \frac{\partial}{\partial \gamma_{2}}-\gamma_{2} \frac{\partial}{\partial \gamma_{1}}+M_{1} \frac{\partial}{\partial M_{2}}-M_{2} \frac{\partial}{\partial M_{1}}
$$

и, как мы позже докажем, входит в разложение $X$ вида (8):

$$
X=P \mathrm{~d} H+\eta X_{S} .
$$

Известно (см., например, [3], [23], [30]), что для векторного поля $X$ (32) в данном случае всегда можно найти пару линейных по импульсам интегралов движения

$$
J_{k}=v_{1}^{(k)}\left(\gamma_{3}\right)\left(\gamma_{1} M_{1}+\gamma_{2} M_{2}\right)+v_{2}^{(k)}\left(\gamma_{3}\right) M_{3}, \quad k=1,2
$$


В общем случае коэффициенты $v_{1}^{(k)}$ и $v_{2}^{(k)}$ являются вещественными аналитическими, но не всегда алгебраическими функциями переменной $\gamma_{3}$, они удовлетворяют следующей системе дифференциальных уравнений:

$$
\begin{aligned}
\frac{g^{2}\left(\left(v_{2}^{\prime}\right)^{(k)}+v_{1}^{(k)}\right)}{m}=m & f_{1} f_{2}\left(\left(1-\gamma_{3}^{2}\right) f_{1}+\gamma_{3} f_{2}\right)\left(v_{2}^{(k)} f_{1}-v_{1}^{(k)} f_{2}\right) \\
+ & I_{1} f_{1}\left(\left(1-\gamma_{3}^{2}\right)\left(v_{1}^{(k)} f_{2}^{\prime}-v_{2}^{(k)} f_{1}^{\prime}\right)-\gamma_{3} v_{1}^{(k)} f_{2}\right. \\
+ & \left.\left(v_{1}^{(k)}\left(\gamma_{3}^{2}-1\right)+\gamma_{3} v_{2}^{(k)}\right) f_{1}\right) \\
\frac{g^{2}\left(v_{1}^{\prime}\right)^{(k)}}{m}=m & f_{1}^{2}\left(\left(1-\gamma_{3}^{2}\right) f_{1}+\gamma_{3} f_{2}\right)\left(v_{2}^{(k)} f_{1}-v_{1}^{(k)} f_{2}\right) \\
& +I_{3}\left(f_{1}^{2} v_{2}^{(k)}-\left(v_{1}^{(k)} f_{2}^{\prime}-v_{2}^{(k)} f_{1}^{\prime}\right) f_{2}\right) .
\end{aligned}
$$

Эта система получена приравниванием нулю коэффициентов при $M_{1,2}$ и $M_{3}$ в уравнениях

$$
\dot{J}_{k}=X\left(J_{k}\right)=0, \quad k=1,2 .
$$

Здесь и всюду далее в этом разделе мы полагаем $(\gamma, \gamma)=1$ и опускаем зависимость функций от $\gamma_{3}$, т. е. используем обозначения

$$
F\left(\gamma_{3}\right)=F, \quad \frac{\mathrm{d} F\left(\gamma_{3}\right)}{\mathrm{d} \gamma_{3}}=F^{\prime}
$$

для всех функций $F$ от переменной $\gamma_{3}$.

Помимо четырех интегралов движения $H, C$ и $J_{1,2}$ векторное поле $(32)$ в данном случае обладает еще инвариантной мерой

$$
\rho=g^{-1}(\gamma) \mathrm{d} \gamma \mathrm{d} M, \quad g(\gamma)=\sqrt{I_{1} I_{3}+m(r, \mathbf{I} r)} .
$$

Таким образом, система (32) для тела вращения является интегрируемой в квадратурах по теореме Эйлера-Якоби.

ПримеР 1. Рассмотрим круглый диск со смещенным вдоль оси динамической симметрии центром масс, при этом

$$
f_{1}=\frac{R}{\sqrt{1-\gamma_{3}^{2}}}, \quad f_{2}=a .
$$

Здесь $R$ - радиус диска (монеты), $a$ - смещение центра масс. Если $a=0$, то функции

$$
v_{1}^{(k)}=L^{(k)}\left(b_{-}, \gamma_{3}\right), \quad v_{2}^{(k)}=c\left(L^{(k)}\left(b_{+}, \gamma_{3}\right)-\gamma_{3} L^{(k)}\left(b_{-}, \gamma_{3}\right)\right), \quad k=1,2,
$$

где

$$
b_{\mp}=\frac{\sqrt{g^{2}-4 m I_{3} R^{2}}}{2 g} \mp \frac{1}{2}, \quad c=-\frac{I_{1}^{1 / 2} \sqrt{g^{2}-4 m I_{3} R^{2}}+g I_{1}}{2 m g R^{2}},
$$

выражаются через функции Лежандра $L^{(1,2)}\left(b_{k}, \gamma_{3}\right)$ первого и второго рода, которые стандартным образом определяются через гипергеометрическую функцию [23].

Если $a \neq 0$, то решения уравнений (76) являются комбинациями функций Уиттекера (см. [8]). 
5.1. Гамильтонизация редуцированной системы. Следуя [23], редуцируем систему по действию (72) группы симметрий $\mathrm{SO}(2)$, выбрав в качестве переменных инвариантные относительно поля симметрий функции

$$
\begin{gathered}
\gamma_{3}, \quad K_{1}=\frac{(M, r)}{f_{1}}, \quad K_{2}=g \omega_{3}, \quad K_{3}=\frac{\kappa\left(\gamma_{1} M_{2}-\gamma_{2} M_{1}\right)}{1-\gamma_{3}^{2}}, \\
\kappa=\sqrt{\frac{1-\gamma_{3}^{3}}{I_{1}+m(r, r)}} .
\end{gathered}
$$

Получим

$$
\begin{aligned}
\dot{\gamma}_{3}= & \kappa K_{3}, \\
\dot{K}_{1}=- & \kappa K_{3} g^{-1} I_{3}\left(1-\left(\frac{f_{2}}{f_{1}}\right)^{\prime}\right) K_{2}, \\
\dot{K}_{2}=- & \kappa K_{3} g^{-1} m f_{1}\left(f_{1}-f_{2}^{\prime}\right) K_{1} \\
\dot{K}_{3}=- & \frac{\kappa}{I_{1}^{2} f_{1}^{2}\left(1-\gamma_{3}^{2}\right)^{2}}\left(f_{2}\left(f_{1}\left(1-\gamma_{3}^{2}\right)+\gamma_{3} f_{2}\right)\left(m f_{1}^{2} K_{1}^{2}+I_{3} K_{2}^{2}\right)\right. \\
& \quad+\gamma_{3} f_{1}^{2} I_{1} K_{1}^{2}+f_{1}\left(f_{1}\left(1-\gamma_{3}^{2}\right)+2 \gamma_{3} f_{2}\right) K_{1} K_{2} g \\
& \left.\quad+m f_{1}^{2} g^{-1} f_{2}\left(1-\gamma_{3}^{2}\right)\left(\gamma_{3} f_{1} I_{1}-f_{2} I_{3}\right) K_{1} K_{2}\right)-\frac{\partial U\left(\gamma_{3}\right)}{\partial \gamma_{3}},
\end{aligned}
$$

при этом интеграл энергии имеет вид

$$
H=\frac{1}{2 I_{1}\left(1-\gamma_{3}^{2}\right)}\left(K_{1}^{2}-\frac{I_{3}}{m f_{1}^{2}} K_{2}^{2}+\frac{m f_{2}^{2}}{I_{1}}\left(K_{1}-\frac{K_{2} g}{m f_{1} f_{2}}\right)^{2}\right)+\frac{1}{2} K_{3}^{2}+U\left(\gamma_{3}\right) .
$$

Векторное поле $\widehat{X}$ приведенной системы (80) является гамильтоновым по отношению к пуассоновой структуре второго ранга:

$$
\widehat{P}=\kappa\left(\begin{array}{cccc}
0 & 0 & 0 & \widehat{X}=\widehat{P} \mathrm{~d} H \\
0 & 0 & 0 & -I_{3} g^{-1}\left(1-\left(f_{2} / f_{1}\right)^{\prime}\right) K_{2} \\
0 & 0 & 0 & -m g^{-1} f_{1}\left(f_{1}-f_{2}^{\prime}\right) K_{1} \\
* & * & * & 0
\end{array}\right), \quad \operatorname{rank} \widehat{P}=2
$$

(звездочками здесь и далее обозначены члены, которые получаются из кососимметричности).

Чтобы найти функции Казимира скобки (82), найдем линейные интегралы системы (80), для этого поделим второе и третье уравнения на первое и получим два линейных неавтономных уравнения

$$
\frac{\mathrm{d} K_{1}}{\mathrm{~d} \gamma_{3}}=-g^{-1} I_{3}\left(1-\left(\frac{f_{2}}{f_{1}}\right)^{\prime}\right) K_{2}, \quad \frac{\mathrm{d} K_{2}}{\mathrm{~d} \gamma_{3}}=-m g^{-1} f_{1}\left(f_{1}-f_{2}^{\prime}\right) K_{1},
$$

которые в других обозначениях были получены Чаплыгиным [30] (см. обсуждение в [23]). 
Общее решение данной системы уравнений всегда существует и имеет вид

$$
K_{i}=c_{1} \Phi_{1}\left(\gamma_{3}\right)+c_{2} \Phi_{2}\left(\gamma_{3}\right), \quad i=1,2,
$$

где $\Phi_{1,2}$ - фундаментальные решения уравнений (83), а константы интегрирования $c_{1,2}$ являются интегралами системы и могут быть выражены через (75). Разрешая полученные соотношения (84) относительно $c_{1,2}$, мы получим эти интегралы движения, которые и являются функциями Казимира бивектора Пуассона $\widehat{P}$.

5.2. Пуассонова структура ранга 4. В отличие от шара Чаплыгина, в этом случае мы имеем явные выражения для механической энергии системы $H$ и геометрического интеграла $C$ (33) и неявные определения для двух линейных по импульсам интегралов движения $J_{1,2}(75)$. Тем не менее, используя линейный по $M_{i}$ анзац (13) и уравнения (76), определяющие функции $v_{1,2}^{(k)}$, мы можем получить решения уравнений (12).

В отличие от волчка Лагранжа, обладающего аналогичными симметриями, в этом случае уравнения (12) имеют два линейных по $M_{i}$ решения $P^{(k)}$, а не три. Эти решения условно можно разделить на "горизонтальную" и "вертикальную" части:

$$
P^{(k)}=\alpha P_{\alpha}^{(k)}+\beta P_{\beta}, \quad k=1,2 .
$$

Здесь $P_{\alpha}^{(k)}$ и $P_{\beta}$ - два согласованных бивектора Пуассона второго ранга,

$$
\left[P_{\alpha}^{(k)}, P_{\beta}\right]=0,
$$

удовлетворяющих уравнениям (12), а $\alpha$ и $\beta$ - произвольные функции от $\gamma_{2} / \gamma_{1}$ и $\gamma_{3}$ соответственно.

Первая составляющая зависит от функций $v_{1,2}^{(k)}$, входящих в определение линейных по импульсам интегралов движения $J_{k}(75)$ :

$$
P_{\alpha}^{(k)}=\zeta_{k}\left(\begin{array}{cc}
0 & \boldsymbol{\Gamma}_{\alpha} \\
-\boldsymbol{\Gamma}_{\alpha}^{\top} & \mathbf{M}_{\alpha}
\end{array}\right), \quad \operatorname{rank} P_{\alpha}^{(k)}=2, \quad k=1,2,
$$

где

$$
\begin{aligned}
\boldsymbol{\Gamma}_{\alpha}= & \left(\begin{array}{cccc}
\frac{\gamma_{1} \gamma_{2}}{\gamma_{1}^{2}+\gamma_{2}^{2}} \frac{v_{2}^{(k)}}{v_{1}^{(k)}} & \frac{\gamma_{2}^{2}}{\gamma_{1}^{2}+\gamma_{2}^{2}} \frac{v_{2}^{(k)}}{v_{1}^{(k)}} & -\gamma_{2} \\
-\frac{\gamma_{1}^{2}}{\gamma_{1}^{2}+\gamma_{2}^{2}} \frac{v_{2}^{(k)}}{v_{1}^{(k)}} & -\frac{\gamma_{1} \gamma_{2}}{\gamma_{1}^{2}+\gamma_{2}^{2}} \frac{v_{2}^{(k)}}{v_{1}^{(k)}} & \gamma_{1} \\
0 & 0 & 0
\end{array}\right), \\
\mathbf{M}_{\alpha}= & \left(\begin{array}{ccc}
0 & \frac{\gamma_{1} M_{1}+\gamma_{2} M_{2}}{\gamma_{1}^{2}+\gamma_{2}^{2}} \frac{v_{2}^{(k)}}{v_{1}^{(k)}} & -M_{2} \\
* & 0 & M_{1} \\
* & * & 0
\end{array}\right), \\
\zeta_{k}= & \exp \left(\int \frac { 1 } { v _ { 1 } ^ { ( k ) } g ^ { 2 } } m \left(m f_{1}^{2}\left(\left(1-\gamma_{3}^{2}\right) f_{2}+\gamma_{3} f_{2}\right)\left(f_{1} v_{2}^{(k)}-f_{2} v_{1}^{(k)}\right)\right.\right. \\
& \left.\left.+I_{1} v_{1}^{(k)} f_{1}\left(\left(1-\gamma_{3}^{2}\right) f_{1}^{\prime}-\gamma_{3} f_{1}\right)+I_{3} v_{2}^{(k)}\left(f_{1}^{\prime} f_{2}-f_{1}^{2}\right)\right) \mathrm{d} \gamma_{3}\right) .
\end{aligned}
$$


Бивекторы Пуассона $P_{\alpha}^{(1)}$ и $P_{\alpha}^{(2)}$ обладают тремя общими функциями Казимира:

$$
P_{\alpha}^{(k)} \mathrm{d} C=0, \quad P_{\alpha}^{(k)} \mathrm{d} \gamma_{3}=0, \quad P_{\alpha}^{(k)} \mathrm{d}\left(\gamma_{1} M_{2}-\gamma_{2} M_{1}\right)=0,
$$

и двумя различными функциями Казимира:

$$
P_{\alpha}^{(1)} \mathrm{d} J_{1}=0, \quad P_{\alpha}^{(2)} \mathrm{d} J_{2}=0,
$$

а также порождают поле симметрий, действуя на $M_{3}$ :

$$
P_{\alpha}^{(k)} \mathrm{d} M_{3}=X_{S} .
$$

Эти бивекторы могут рассматриваться как "вертикальная" составляющая полного решения (85), которая пропадает при редукции по оставшейся $\mathrm{SO}(2)$-симметрии.

Вторая часть решения (85) имеет вид

$$
P_{\beta}=\left(\begin{array}{cc}
0 & \boldsymbol{\Gamma}_{\beta} \\
-\boldsymbol{\Gamma}_{\beta}^{\top} & \mathbf{M}_{\beta}
\end{array}\right), \quad \operatorname{rank} P_{\beta}=2,
$$

где

$$
\begin{aligned}
& \boldsymbol{\Gamma}_{\beta}=\left(\begin{array}{ccc}
\frac{\gamma_{1} \gamma_{2} \gamma_{3}}{\gamma_{1}^{2}+\gamma_{2}^{2}} & -\frac{\gamma_{1}^{2} \gamma_{3}}{\gamma_{1}^{2}+\gamma_{2}^{2}} & 0 \\
\frac{\gamma_{2}^{2} \gamma_{3}}{\gamma_{1}^{2}+\gamma_{2}^{2}} & -\frac{\gamma_{1} \gamma_{2} \gamma_{3}}{\gamma_{1}^{2}+\gamma_{2}^{2}} & 0 \\
-\gamma_{2} & \gamma_{1} & 0
\end{array}\right) \text {, } \\
& \mathbf{M}_{\beta}=\left(\begin{array}{ccc}
0 & -M_{3}+\frac{\gamma_{3}\left(\gamma_{1} M_{1}+\gamma_{2} M_{2}\right)}{\gamma_{1}^{2}+\gamma_{2}^{2}}+m \tau & -m \sigma \gamma_{2} \\
* & 0 & m \sigma \gamma_{1} \\
* & * & 0
\end{array}\right) \text {, } \\
& \tau=\frac{f_{2}}{f_{1}} \sigma-\frac{f_{1}\left(I_{3} f_{2}\left(\gamma_{1} M_{1}+\gamma_{2} M_{2}\right)-\left(\gamma_{1}^{2}+\gamma_{2}^{2}\right) I_{1} f_{1} M_{3}\right)}{g^{2}}\left(1-\left(\frac{f_{2}}{f_{1}}\right)^{\prime}\right), \\
& \sigma=\frac{1}{g^{2}}\left[\left(\gamma_{1} M_{1}+\gamma_{2} M_{2}\right)\left(m(r, \gamma) f_{1}^{3}+I_{3}\left(f_{1}^{2}+f_{1}^{\prime} f_{2}\right)\right)+M_{3}\left(m(r, \gamma) f_{1}^{2} f_{2}\right.\right. \\
& \left.\left.+\left(\gamma_{3} f_{1}-\left(\gamma_{1}^{2}+\gamma_{2}^{2}\right) f_{1}^{\prime}\right) I_{1} f_{1}\right)\right] .
\end{aligned}
$$

Бивектор Пуассона второго ранга $P_{\beta}$ обладает следующими функциями Казимира:

$$
P_{\beta} \mathrm{d} C=0, \quad P_{\beta} \mathrm{d} J_{1}=0, \quad P_{\beta} \mathrm{d} J_{2}=0, \quad P_{\beta} \mathrm{d} \frac{\gamma_{2}}{\gamma_{1}}=0 .
$$

Заметим, что эта пуассонова структура зависит только от формы тела вращения (т. е. только от функций $f_{1,2}$ ), она может рассматриваться как "горизонтальная" составляющая полного решения уравнений (12), которая сохраняется при редукции по оставшейся симметрии системы $X_{S}$ и переходит в скобку (82).

Формально бивекторы $P^{(k)}$ определены локально, так как $\mathbf{M}_{\alpha}$ и $\mathbf{M}_{\beta}$ имеют особенности при

$$
\gamma_{1,2}=0, \quad \gamma_{3}= \pm 1, \quad M_{1,2} \neq 0
$$


Особенность в элементе матрицы $\mathbf{M}_{\alpha}$

$$
\frac{\gamma_{1} M_{1}+\gamma_{2} M_{2}}{\gamma_{1}^{2}+\gamma_{2}^{2}} \frac{v_{2}^{(k)}}{v_{1}^{(k)}}
$$

можно убрать, выбирая интегралы движения таким образом, чтобы $v_{2}^{(k)}=0$ при $\gamma_{3}=1$ или при $\gamma_{3}=-1$. С другой стороны, бивектор $P_{\beta}$, используя свободу выбора функции $\beta$, можно умножить на

$$
\beta=1-\gamma_{3}^{2}
$$

и тем самым получить общее решение без особенностей.

Таким образом, из предыдущего следует, что на регулярной части пуассонова многообразия каждый из бивекторов $P^{(k)}(85)$ обладает двумя функциями Казимира:

$$
P^{(k)} \mathrm{d} C=0, \quad P^{(k)} \mathrm{d} J_{k}=0, \quad k=1,2 .
$$

При этом равенство нулю скобок Схоутена эквивалентно существованию линейных интегралов движения $J_{1,2}(75)$ :

$$
\left[P^{(k)}, P^{(k)}\right]=0 \quad \Leftrightarrow \quad \dot{J}_{k}=X\left(J_{k}\right)=0 .
$$

Полученная нами пуассонова структура на частично редуцированном фазовом пространстве позволяет нам построить конформно-гамильтоново разложение исходного векторного поля $X$.

ПреДложениЕ 5. Для тела вращения на плоскости векторное поле X (32) является линейной комбинацией гамильтонова векторного поля и поля симметрий $X_{S}(74)$ :

$$
X=\beta^{-1} P^{(k)} \mathrm{d} H+\eta_{k} X_{S}, \quad \eta_{k}=\frac{u_{1}\left(\gamma_{1} M_{1}+\gamma_{2} M_{2}\right)+u_{2} M_{3}}{g^{2}},
$$

где коэфбициенть

$$
\begin{aligned}
u_{1}= & \frac{1}{\left(1-\gamma_{3}^{2}\right) v_{1}^{(k)}}\left[m f_{1}\left(1-\gamma_{3}^{2}\right)\left(\beta^{-1} \alpha \zeta_{k}\left(v_{1}^{(k)} f_{2}-v_{2}^{(k)} f_{1}\right)+v_{1}^{(k)}\left(\gamma_{3} f_{1}-f_{2}\right)\right)\right. \\
& \left.\quad+I_{3}\left(\gamma_{3} v_{1}^{(k)}+\beta^{-1} \alpha \zeta_{k} v_{2}^{(k)}\right)\right] \\
u_{2}= & \frac{1}{v_{1}^{(k)}}\left[m f_{2}\left(\beta^{-1} \alpha \zeta_{k}\left(v_{1}^{(k)} f_{2}-v_{2}^{(k)} f_{1}\right)+v_{1}^{(k)}\left(\gamma_{3} f_{1}-f_{2}\right)\right)-I_{1} v_{1}^{(k)}\left(\beta^{-1} \alpha \zeta_{k}+1\right)\right]
\end{aligned}
$$

зависят от функциц $v_{1,2}^{(k)}$, входящих в определение интегралов движения $J_{k}(75)$.

Воспользуемся теперь свободой в выборе функций $\alpha$ и $\beta$ в решении (85). Если положить $\alpha=$ const и

$$
\beta=-\frac{\left(1-\gamma_{3}^{2}\right)\left(f_{1} v_{2}^{(k)}-f_{2} v_{1}^{(k)}\right)^{2} m+I_{1}\left(1-\gamma_{3}^{2}\right)\left(v_{1}^{(k)}\right)^{2}+I_{3}\left(v_{2}^{(k)}\right)^{2}}{\left(1-\gamma_{3}^{2}\right)\left(\gamma_{3} f_{1}-f_{2}\right)\left(f_{1} v_{2}^{(k)}-f_{2} v_{1}^{(k)}\right) m+I_{1}\left(1-\gamma_{3}^{2}\right) v_{1}^{(k)}+I_{3} v_{2}^{(k)}} \frac{\alpha \zeta_{k}}{v_{1}^{(k)}},
$$


то векторное поле $X$ будет иметь вид

$$
X=\beta^{-1} P^{(k)} \mathrm{d} H+\delta J_{k} X_{S},
$$

где

$$
\delta=\frac{\gamma_{3} v_{1}^{(k)}-v_{2}^{(k)}}{\left(1-\gamma_{3}^{2}\right)\left(f_{1} v_{2}^{(k)}-f_{2} v_{1}^{(k)}\right)^{2} m+I_{1}\left(1-\gamma_{3}^{2}\right)\left(v_{1}^{(k)}\right)^{2}+I_{3}\left(v_{2}^{(k)}\right)^{2}} .
$$

ПредложениЕ 6. Для тела вращения на плоскости векторное поле $X$ (32) является конформно-гамильтоновым,

$$
X=\beta^{-1} P^{(k)} \mathrm{d} H,
$$

относительно бивектора Пуассона четвертого ранга при нулевом значении одного из линейных по моментам интегралов движения:

$$
J_{k}=0, \quad k=1,2 .
$$

Таким образом, после замены времени $X$ приводится к гамильтонову векторному полю при нулевом значении одной из функций Казимира бивектора $P^{(k)}$.

5.3. Приведение скобок Пуассона к каноническим. Как и для шара Чаплыгина, рассмотрим преобразования переменных вида

$$
\begin{aligned}
& L_{1}=\frac{1}{\gamma_{1}^{2}+\gamma_{2}^{2}}\left(\frac{\gamma_{1} \gamma_{3}}{\alpha}\left(\frac{v_{1}^{(k)}\left(\gamma_{1} M_{1}+\gamma_{2} M_{2}\right)}{v_{2}^{(k)} \zeta_{k}}+b \varrho_{k}\right)+\frac{\gamma_{2}\left(\gamma_{1} M_{2}-\gamma_{2} M_{1}\right)}{\beta}+c \gamma_{1}\right), \\
& L_{2}=\frac{1}{\gamma_{1}^{2}+\gamma_{2}^{2}}\left(\frac{\gamma_{2} \gamma_{3}}{\alpha}\left(\frac{v_{1}^{(k)}\left(\gamma_{1} M_{1}+\gamma_{2} M_{2}\right)}{v_{2}^{(k)} \zeta_{k}}+b \varrho_{k}\right)-\frac{\gamma_{1}\left(\gamma_{1} M_{2}-\gamma_{2} M_{1}\right)}{\beta}+c \gamma_{2}\right), \\
& L_{3}=\frac{M_{3}}{\alpha \zeta_{k}}-\frac{1+\zeta_{k} \varrho_{k} v_{2}^{(k)}}{\alpha \zeta_{k} v_{2}^{(k)}} b, \quad b=J_{k}, \quad c=(L, \gamma),
\end{aligned}
$$

где

$$
\varrho_{k}=\int \frac{v_{1}^{(k)} \mu_{k}}{\left(v_{2}^{(k)}\right)^{2} \zeta_{k}} \mathrm{~d} \gamma_{3}
$$

и

$$
\mu_{k}=1-m g^{-2}\left(1-\gamma_{3}^{2}\right)\left(I_{1}+m f_{2}^{2}\right) f_{1}^{2}+m g^{-2}\left(m \gamma_{3} f_{2}^{3}-I_{1}\left(1-\gamma_{3}^{2}\right) f_{2}^{\prime}+I_{1} \gamma_{3} f_{2}\right) f_{1} .
$$

Легко проверить, что если $\alpha=$ const, то отображения Пуассона (91)

$$
\psi_{k}:(\gamma, M) \rightarrow(\gamma, L)
$$

приводят скобки Пуассона $\{\cdot, \cdot\}$, отвечающие бивекторам $P^{(k)}(85)$, к каноническим скобкам Ли-Пуассона (9) на алгебре Ли $e^{*}(3)$. Подчеркнем, что эти отображения в общем случае корректно определены только на регулярной части рассматриваемых пуассоновых многообразий и не определены в сингулярных точках $e_{ \pm}$, где $\operatorname{rank} P^{(k)}=2$. 
При

$$
c=(\gamma, L)=0
$$

мы имеем пуассоновы отображения, отождествляющие наше частично редуцированное шестимерное фазовое пространство с кокасательным расслоением двумерной сферы $T^{*} S$ с каноническими скобками Пуассона. Для бивектора Пуассона $P^{(1)}$ с функцией Казимира $J_{1}(75)$,

$$
P^{(1)} \mathrm{d} J_{1}=0,
$$

интегралы движения $H$ и $J_{2}$ после замены переменных примут вид

$$
\begin{aligned}
& H=A L_{3}^{2}+B\left(L_{1} \gamma_{2}-L_{2} \gamma_{1}\right)^{2}+b C L_{3}+b^{2} D \\
& J_{2}=\frac{\zeta_{1}\left(v_{1}^{(1)} v_{2}^{(2)}-v_{1}^{(2)} v_{2}^{(1)}\right)}{v_{1}^{(1)}}\left(\alpha L_{3}+b \varrho_{1}\right)+\frac{v_{2}^{(2)}}{v_{2}^{(1)}} b,
\end{aligned}
$$

где $A, B, C$ и $D$ - функции от $\gamma_{3}$.

5.4. Гиростатические обобщения. Напомним, что при добавлении к катящемуся по плоскости телу ротора, который вращается с постоянной угловой скоростью, уравнения движения (32) изменятся следующим образом:

$$
\dot{M}=(M+S) \times \omega+m \dot{r} \times(\omega \times r)+M_{F}, \quad \dot{\gamma}=\gamma \times \omega,
$$

где $S$ - постоянный трехмерный вектор гиростатического момента.

Для шара Чаплыгина на плоскости этот вектор произволен, а для тела вращения на плоскости интегрируемость сохраняется тогда и только тогда, когда гиростатический момент

$$
S=(0,0, s)
$$

направлен вдоль оси симметрии. В этом случае линейные по импульсам интегралы движения (75) просто "сдвигаются" на линейное по $s$ слагаемое:

$$
J_{k}^{(s)}=J_{k}+s \int v_{1}^{(k)} \mathrm{d} \gamma_{3}, \quad k=1,2 .
$$

Здесь $v_{1}^{(k)}$ - функции переменной $\gamma_{3}$, определяющие интегралы движения $J_{k}(75)$ для тела без ротора. Заметим, что в этом случае даже для диска из примера 1 мы уже получим неалгебраические интегралы движения, так как при интегрировании $\int L^{(1,2)}\left(b_{-}, \gamma_{3}\right) \mathrm{d} \gamma_{3}$ функций Лежандра $L^{(1,2)}$ мы получим неалгебраические выражения.

Решения уравнений (12) в этом случае будут просто "сдвигами" уже полученных нами решений:

$$
P_{s}^{(k)}=P^{(k)}-\beta\left(\begin{array}{ll}
0 & 0 \\
0 & \mathbf{S}
\end{array}\right),
$$

т. е. сдвигом бивектора второго ранга $P_{\beta}$. В этом случае векторное поле

$$
X=\beta^{-1} P_{s}^{(k)} \mathrm{d} H+\eta_{k} X_{S}, \quad k=1,2,
$$

по-прежнему будет линейной комбинацией гамильтонова поля и поля симметрий с теми же самыми коэффициентами $\eta_{k}$, что для тела вращения без ротора. 
5.5. Сфера Рауса. В качестве примера рассмотрим качение сферы Рауса по плоскости [9], [23], [32], [33], [44], [74]. В этом случае центр масс сферы не совпадает с ее геометрическим центром, а ось, проходящая через центр масс и геометрический центр, является осью динамической симметрии, т. е. два главных момента инерции относительно осей, перпендикулярных ей, равны друг другу. Сфера катится по горизонтальной плоскости под действием вертикально направленной постоянной силы тяжести.

В этом случае форма тела вращения задается функциями $f_{1}=R$ и $f_{2}=$ $R \gamma_{3}+a$, т. е.

$$
r=\left(R \gamma_{1}, R \gamma_{2}, R \gamma_{3}+a\right),
$$

где $R$ - радиус сферы, $a$ - расстояние от геометрического центра до центра масс.

Подставляя функции $f_{1,2}$ в уравнения (76), мы легко найдем линейные по моментам интегралы движения $J_{1,2}(75)$. Первый из них,

$$
J_{1}=(M, r),
$$

- это интеграл Джеллета [47], см. также §243 в книге Рауса [75]. Второй интеграл движения,

$$
J_{2}=g(\gamma) \omega_{3}
$$

был найден Э. Раусом в 1884 г. [75] и затем переоткрыт С. А. Чаплыгиным [30]. Напомним, что $\omega_{3}$ - третья компонента вектора угловой скорости, которая выражается через угловой момент согласно $(32)$, а $g \equiv g(\gamma)$ - функция, определяющая плотность инвариантной меры (77).

Так как $v_{1}^{(k)}$ входят также и в определение бивекторов Пуассона, мы их выпишем явно:

$$
\begin{array}{lll}
v_{1}^{(1)}=f_{1}=R, & v_{2}^{(1)}=f_{2}=R \gamma_{3}+a, & \zeta_{1}=g, \\
v_{1}^{(2)}=\frac{m R\left(R \gamma_{3}+a\right)}{g}, & v_{2}^{(2)}=\frac{I_{1}+m\left(R \gamma_{3}+a\right)^{2}}{g}, & \zeta_{2}=R \gamma_{3}+a .
\end{array}
$$

Соответствующие бивекторы Пуассона $P^{(1,2)}(85)$ были найдены в [9]. Используя эти бивекторы Пуассона, мы можем построить различные представления векторного поля $X(32)$ :

$$
X=\beta^{-1} P^{(1)} \mathrm{d} H+\eta_{1} X_{S}=\beta^{-1} P^{(2)} \mathrm{d} H+\eta_{2} X_{S}
$$

или

$$
X=\beta^{-1} P^{(1)} \mathrm{d} H+\frac{\alpha I_{1}}{g^{2}} \eta_{1} P^{(1)} \mathrm{d} J_{2}=\beta^{-1} P^{(2)} \mathrm{d} H-\frac{\alpha I_{1}}{m g^{2}} \eta_{2} P^{(2)} \mathrm{d} J_{1} .
$$

ЗАмечАние 13. Если вместо интегралов Джеллета $J_{1}$ и Рауса $J_{2}$ взять их линейные комбинации

$$
\begin{aligned}
& J_{+}=J_{1}-\frac{\sqrt{I_{3}}(R+a)}{\sqrt{m(R+a)^{2}+I_{1}}} J_{2}=v_{1}^{(+)}\left(\gamma_{1} M_{1}+\gamma_{2} M_{2}\right)+v_{2}^{(+)} M_{3}, \\
& J_{-}=J_{1}+\frac{\sqrt{I_{3}}(R-a)}{\sqrt{m(R-a)^{2}+I_{1}}} J_{2}=v_{1}^{(-)}\left(\gamma_{1} M_{1}+\gamma_{2} M_{2}\right)+v_{2}^{(-)} M_{3}
\end{aligned}
$$


и использовать их в качестве функций Казимира для бивекторов $P_{\alpha}^{( \pm)}$, построенных по правилу (86), то эти бивекторы не будут иметь особенностей в точках $\gamma_{3}=1$ и $\gamma_{3}=-1$ соответственно. Подчеркнем, что для сферы Рауса геометрический центр не совпадает с центом тяжести и поэтому для этой динамической системы положения равновесия (73) будут различными - одно положение устойчиво, а второе нет. Выбирая подходящим образом линейные интегралы движения $J_{ \pm}$, можно построить бивекторы $P_{\alpha}^{( \pm)}(86)$, которые будут иметь особенности только в одном из положений равновесия.

Обсуждение различных приложений общей теории сингулярностей на пуассоновых многообразиях для изучения динамических систем может быть найдено в работе [67] и в приведенных в ней ссылках. Использованию особенностей бигамильтоновых структур при анализе устойчивости периодических решений посвящена работа [15]. В работе [14] исследуются топологические препятствия (негамильтонова монодромия) к гамильтонизации неголономных систем.

Теперь рассмотрим отображение Пуассона (91), приводящее скобки к канонической форме. Для примера мы возьмем скобки, для которых интеграл Джеллета (97) будет функцией Казимира. В этом случае

$$
\varrho_{1}=-\frac{I_{1}+m\left(R \gamma_{3}+a\right)^{2}}{g I_{1}\left(R \gamma_{3}+a\right)}
$$

и при $\alpha=$ const и $c=0$ после отображения Пуассона функция Гамильтона

$$
\begin{gathered}
H=\frac{1}{2 R^{2}\left(1-\gamma_{3}^{2}\right)}\left(\alpha^{2} L_{3}^{2}\left(I_{1} R^{2}\left(\gamma_{3}^{2}-1\right)-\left(R \gamma_{3}+a\right)^{2} I_{3}\right)+\frac{\beta^{2}\left(\gamma_{2} L_{1}-\gamma_{1} L_{2}\right)^{2}}{\left(I_{1}+m(r, r)\right)}\right. \\
\left.-\frac{2 \alpha g b\left(r \gamma_{3}+a\right) L_{3}}{I_{1}}+\frac{I_{1}+m\left(R \gamma_{3}+a\right)^{2} b^{2}}{I_{1}^{2}}\right)+U\left(\gamma_{3}\right)
\end{gathered}
$$

и интеграл Рауса

$$
J_{2}=\alpha I_{1} L_{3}
$$

будут определять динамическую систему на двумерной сфере с каноническими скобками Пуассона. Используя стандартные сферические координаты

$$
\begin{array}{ll}
\gamma_{1}=\sin \phi \sin \theta, & L_{1}=\frac{\sin \phi \cos \theta}{\sin \theta} p_{\phi}-\cos \phi p_{\theta}, \\
\gamma_{2}=\cos \phi \sin \theta, & L_{2}=\frac{\cos \phi \cos \theta}{\sin \theta} p_{\phi}+\sin \phi p_{\theta}, \\
\gamma_{3}=\cos \theta, & L_{3}=-p_{\phi},
\end{array}
$$

где $\phi, \theta$ - углы Эйлера, $p_{\phi}, p_{\theta}$ - сопряженные импульсы:

$$
\left\{\phi, p_{\phi}\right\}=\left\{\theta, p_{\theta}\right\}=1, \quad\{\phi, \theta\}=\left\{\phi, p_{\theta}\right\}=\left\{\theta, p_{\phi}\right\}=0,
$$

мы можем переписать интегралы движения в следующем стандартном виде:

$$
H=\frac{A(\theta) p_{\phi}^{2}+B(\theta) p_{\theta}^{2}+b C(\theta) p_{\phi}+b^{2} D(\theta)}{2}+U(\theta), \quad J_{2}=-\alpha I_{1} p_{\phi} .
$$


Здесь $b$ - значение интеграла Джеллета $J_{1}, \alpha=$ const и

$$
\begin{aligned}
& A(\theta)=\alpha^{2}\left(I_{1}+\frac{I_{3}\left(a^{2}+2 a R \cos \theta+R^{2} \cos ^{2} \theta\right)}{R^{2} \sin ^{2} \theta}\right), \\
& B(\theta)=\frac{\beta^{2}}{I_{1}+m\left(a^{2}+2 a R \cos \theta+R^{2}\right)} \\
& C(\theta)=\frac{2 \alpha g(R \cos \theta+a)}{I_{1} R^{2} \sin ^{2} \theta} \\
& D(\theta)=\frac{I_{1}+m\left(a^{2}+2 a R \cos \theta+R^{2}\right)}{I_{1}^{2} R^{2} \sin ^{2} \theta}
\end{aligned}
$$

Уравнение для угла нутации является гамильтоновым:

$$
\dot{\theta}=\{H, \theta\}+\eta\left\{J_{2}, \theta\right\} \equiv\{H, \theta\},
$$

и, полностью аналогично волчку Лагранжа, легко найти квадратуру для угла нутации:

$$
\dot{\theta}=B(\theta) p_{\theta}=\sqrt{B(\theta)\left(2 E_{1}-A(\theta) E_{2}^{2}-b E_{2} C(\theta)-b^{2} D(\theta)-2 U(\theta)\right)},
$$

где $E_{1}=H$ и $E_{2}=-J_{2} /\left(\alpha I_{1}\right)$ - фиксированные значения интегралов движения. Подставляя полученное решение в уравнение для угла собственного вращения, которое не будет гамильтоновым, мы получим вторую квадратуру

$$
\dot{\phi}=\{H, \phi\}+\eta\left\{J_{2}, \phi\right\}=\frac{\left(I_{1} \sin ^{2} \theta+I_{3} \cos ^{2} \theta\right)+a I_{3} \cos \theta}{g(\theta) I_{1} R \sin ^{2} \theta} d-\frac{\cos \theta}{I_{1} R \sin ^{2} \theta} b .
$$

Конечно, эти уравнения можно получить и без нахождения пуассоновых структур и приведения их к канонической форме. Однако исследование пуассоновой геометрии этой системы позволяет прояснить различные нелокальные свойства поведения траекторий в фазовом пространстве, так, например, расслоение на инвариантные многообразия в данном случае оказывается чисто гамильтоновым (лиувиллево слоение) и т. п.

Гиростатическое обобщение интеграла Джеллета останется алгебраической функцией:

$$
J_{1}^{s}=J_{1}+s R \gamma_{3},
$$

в то время как к интегралу Рауса добавится неалгебраическое слагаемое:

$$
J_{2}^{s}=J_{2}+s\left(\frac{\arctan \left(\frac{\sqrt{m}\left(R I_{1} \gamma_{3}-\left(R \gamma_{3}-a\right) I_{3}\right)}{g \sqrt{I_{1}-I_{3}}}\right) a}{\left(I_{1}-I_{3}\right)^{3 / 2} \sqrt{m} R^{2}}-\frac{g}{\left(I_{1}-I_{3}\right) m I_{1} R^{2}}\right) .
$$

Напомним, что этот интеграл является функцией Казимира для бивектора Пуассона $P_{s}^{(2)}(96)$, элементы которого по-прежнему являются алгебраическими функциями. 


\section{6. Динамически симметричный шар на поверхности вращения}

Рассмотрим теперь качение шара, обладающего массой $m$, радиусом $R$ и тензором инерции $\mathbf{I}=\mu \mathbf{E}$, где $\mathbf{E}-$ единичная матрица, по заданной неподвижной поверхности, на форму которой накладывается ограничение, состоящее в том, что шар всегда касается поверхности только в одной точке контакта.

В данном случае оказывается удобным использовать неподвижную систему координат, при этом форма поверхности задается уравнением

$$
\tilde{\mathfrak{f}}(r)=0 .
$$

Так как движение происходит без проскальзывания, то скорость точки контакта обращается в нуль:

$$
v+\omega \times a=0
$$

где $\omega$ и $v$ - угловая скорость и скорость центра шара, $a$ - вектор, соединяющий центр шара и точку контакта (см. рис. 3).

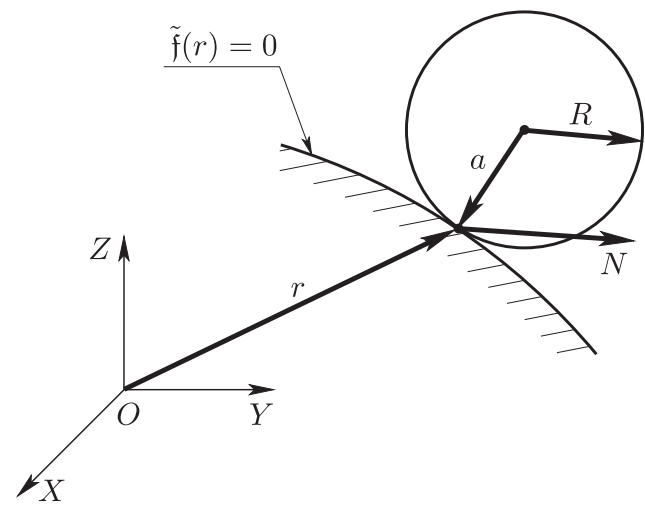

Рис. 3

Пусть $N$ - сила реакции связи (103), $F$ и $M_{F}$ - равнодействующая внешних сил, приложенных к телу, и ее момент, тогда законы сохранения количества движения и углового момента количества движения имеют вид

$$
m \dot{v}=N+F, \quad \dot{\mathbf{I}} \omega=a \times N+M_{F} .
$$

Обозначим через $\gamma$ вектор нормали к поверхности, тогда $a=-R \gamma$, а вместо угловой скорости $\omega$ будем использовать вектор углового момента относительно точки контакта:

$$
M=\mu \omega+d \gamma \times(\omega \times \gamma), \quad d=m R^{2} .
$$

Затем, используя связь (103), исключим из этих уравнений скорость центра масс шара $v$ и реакцию связи $N$ и получим следующую систему динамических уравнений:

$$
\dot{M}=d \dot{\gamma} \times(\omega \times \gamma)+M_{F}, \quad \dot{r}+R \dot{\gamma}=\omega \times R \gamma
$$


которая определяет векторное поле $X$ на шестимерном фазовом пространстве с координатами $x=\left(\gamma_{1}, \gamma_{2}, \gamma_{3}, M_{1}, M_{2}, M_{3}\right)$. Входящий в эти уравнения вектор $r$, определяющий координаты точки контакта, находится с помощью уравнения (102):

$$
\gamma=\frac{\nabla \tilde{\mathfrak{f}}(r)}{|\nabla \tilde{\mathfrak{f}}(r)|} .
$$

Систему (104) необходимо дополнить уравнениями, определяющими ориентацию шара:

$$
\dot{e}_{1}=\omega \times e_{1}, \quad \dot{e}_{2}=\omega \times e_{2}, \quad \dot{e}_{3}=\omega \times e_{3},
$$

где $e_{i}$ - орты осей, связанных с телом, в неподвижной системе координат.

В случае потенциальных внешних сил $F$, порождаемых потенциалом $U=$ $U(r+R \gamma)$, момент внешних сил имеет вид

$$
M_{F}=R \gamma \times \frac{\partial U}{\partial r^{\prime}}, \quad r^{\prime}=r+R \gamma,
$$

а векторное поле $X$ (104) обладает первыми интегралами

$$
H=\frac{1}{2}(M, \omega)+U\left(r^{\prime}\right), \quad C=(\gamma, \gamma)=1 .
$$

Здесь радиус-вектор $r^{\prime}$ задает поверхность, по которой движется центр шара. (Напомним, что все векторы в данном случае записываются в неподвижных осях.)

В общем случае произвольной поверхности группа симметрий $G=\mathrm{SO}(3)$ в этом случае состоит из всевозможных вращений шара. Компоненты векто-

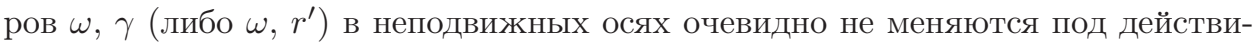
ем этой группы и образуют замкнутый набор инвариантов. Вследствие этого уравнения (104) отделяются от общей системы и образуют редуцированную систему.

Следуя [69], [75], рассмотрим качение однородного шара по поверхности вращения под действием потенциальных внешних сил, направленных вдоль оси симметрии, например, силы тяжести. В этом случае к симметриям системы относительно поворотов шара добавляются повороты поверхности вращения относительно ее оси симметрии [95]. Таким образом, в этом случае полная группа симметрий есть $G=\mathrm{SO}(3) \times \mathrm{SO}(2)$, а редуцированное векторное поле $X$ (104) инвариантно относительно поворотов вокруг оси $O Z$.

В дальнейшем более удобным оказывается вместо поверхности качения рассматривать поверхность, по которой движется центр шара, определяемый радиус-вектором $r^{\prime}=r+R \gamma$. Ее параметризацию в осесимметричном случае можно задать следующим образом:

$$
r_{1}=(f-R) \gamma_{1}, \quad r_{2}=(f-R) \gamma_{2}, \quad r_{3}=\int\left(f-\left(1-\gamma_{3}^{2}\right) g_{3}^{-1} f^{\prime}\right) \mathrm{d} \gamma_{3}-R \gamma_{3}
$$

(см. детальное обсуждение в работе [27]). Функция $f=f\left(\gamma_{3}\right)$ предполагается достаточно выпуклой, чтобы поверхность имела только одну точку контакта с шаром. 
Векторное поле $X$ (104) инвариантно относительно подгруппы симметрий $\mathrm{SO}(2)$, порождаемой полем симметрий

$$
X_{S}=\gamma_{1} \frac{\partial}{\partial \gamma_{2}}-\gamma_{2} \frac{\partial}{\partial \gamma_{1}}+M_{1} \frac{\partial}{\partial M_{2}}-M_{2} \frac{\partial}{\partial M_{1}} .
$$

Кроме этого, векторное поле $X$ обладает инвариантной мерой

$$
f^{3}\left(f-\left(1-\gamma_{3}^{2}\right) \gamma_{3}^{-1} f^{\prime}\right) \mathrm{d} \gamma \mathrm{d} M
$$

Как и при качении тела вращения по плоскости, в данном случае существуют линейные по $M$ интегралы поля $X$

$$
J_{k}=v_{1}^{(k)}\left(\gamma_{1} M_{1}+\gamma_{2} M_{2}\right)+v_{2}^{(k)} M_{3}, \quad k=1,2,
$$

где функции $v_{1,2}\left(\gamma_{3}\right)$, входящие в определение интегралов, удовлетворяют уравнениям

$$
\begin{aligned}
& v_{1}^{\prime}=\frac{f^{\prime}\left(v_{1}\left(\mu+\gamma_{3}^{2} d\right)-\gamma_{3} v_{2} d\right)}{f(\mu+d)}, \\
& v_{2}^{\prime}=v_{1}-\frac{f^{\prime}\left(1-\gamma_{3}^{2}\right)\left(v_{1}\left(\mu+\gamma_{3}^{2} d\right)-\gamma_{3} v_{2} d\right)}{\gamma_{3} f(\mu+d)} .
\end{aligned}
$$

Эта система получена из уравнений

$$
\dot{J}_{k}=X\left(J_{k}\right)=0, \quad k=1,2,
$$

приравниванием нулю коэффициентов при $M_{1,2}$ и $M_{3}$ соответственно. Вещественные аналитические решения уравнений (108) всегда существуют, но не всегда являются алгебраическими функциями.

ПримеР 2. Пусть центр шара движется по эллипсоиду вращения, тогда

$$
f\left(\gamma_{3}\right) \equiv f=-\frac{b_{1}}{b_{1}\left(1-\gamma_{3}^{2}\right)+b_{3} \gamma_{3}^{2}},
$$

где $b_{1,2}^{1 / 2}$ - главные полуоси эллипсоида. При этом уравнения (108) имеют следующие независимые решения:

$$
\begin{gathered}
v_{1}^{(1,2)}=b_{1}^{-1} \gamma_{3}^{3 / 2} f L^{(1,2)}\left(\nu-\frac{1}{2}, \frac{3}{2}, \frac{\sqrt{b_{1}}}{f}\right) \\
v_{2}^{(1,2)}=\gamma_{3}^{1 / 2} f\left(\frac{\gamma_{3}^{2}-1}{b_{1}}+\frac{1}{\left(b_{1}-f^{2}\right)(\nu+1)}\right) L^{(1,2)}\left(\nu-\frac{1}{2}, \frac{3}{2},-\frac{\sqrt{b_{1}}}{f}\right) \\
\quad+\frac{\gamma_{3}^{1 / 2} f^{2}}{\sqrt{b_{1}}\left(b_{1}-f^{2}\right)(\nu+1)} L^{(1,2)}\left(\nu+\frac{1}{2}, \frac{3}{2},-\frac{\sqrt{b_{1}}}{f}\right) .
\end{gathered}
$$

Здесь $L^{(1,2)}$ - присоединенные функции Лежандра первого и второго рода, которые могут быть выражены через гипергеометрическую функцию, и

$$
\nu=\sqrt{\frac{\mu}{\mu+d}} .
$$


Отметим, что, согласно [27], в этом случае существует также алгебраический интеграл

$$
H_{2}=\frac{b_{1}^{2} \mu(\gamma, M)^{2}}{\mu+d}+\left(b_{1}-b_{2}\right)\left(\frac{f \mu\left(\gamma_{3}(\gamma, M)-M_{3}\right)}{\mu+d}\right)^{2},
$$

что позволяет легко получить решения уравнений движения.

Таким образом, в данном случае система уравнений (104) допускает четыре независимых интеграла (105), (107) и инвариантную меру (106), следовательно, она является интегрируемой по теореме Эйлера-Якоби [19], [53].

6.1. Скобки Пуассона для шара на поверхности вращения. Как и для тела вращения на плоскости, подставим в уравнения (12) анзац (13) и составим систему уравнений на функции $c_{i j}^{k}$ и $d_{i j}$, приравнивая нулю коэффициенты при $M_{i}$. Решая полученную переопределенную систему алгебро-дифференциальных уравнений, мы получим два решения

$$
P^{(k)}=\alpha P_{\alpha}^{(k)}+\beta P_{\beta}, \quad \operatorname{rank} P^{(k)}=4, \quad k=1,2 .
$$

Здесь $P_{\alpha}^{(k)}$ и $P_{\beta}$ - два совместных бивектора Пуассона второго ранга,

$$
\left[P_{\alpha}^{(k)}, P_{\beta}\right]=0
$$

удовлетворяющих уравнениям (12), а коэффициенты $\alpha$ и $\beta$ являются произвольными функциями от $\gamma_{2} / \gamma_{1}$ и $\gamma_{3}$ соответственно, т.е., как и ранее, $\alpha=$ $\alpha\left(\gamma_{2} / \gamma_{1}\right)$ и $\beta=\beta\left(\gamma_{3}\right)$.

Первая часть решений (110) имеет ту же форму, что и бивекторы $P_{\alpha}^{(k)}(86)$ для тела вращения на плоскости:

$$
P_{\alpha}^{(k)}=\zeta_{k}\left(\begin{array}{cc}
0 & \boldsymbol{\Gamma}_{\alpha} \\
-\boldsymbol{\Gamma}_{\alpha}^{\top} & \mathbf{M}_{\alpha}
\end{array}\right), \quad \operatorname{rank} P_{\alpha}^{(k)}=2, \quad k=1,2,
$$

где

$$
\begin{aligned}
& \boldsymbol{\Gamma}_{\alpha}=\left(\begin{array}{cccc}
\frac{\gamma_{1} \gamma_{2}}{\gamma_{1}^{2}+\gamma_{2}^{2}} \frac{v_{2}^{(k)}}{v_{1}^{(k)}} & \frac{\gamma_{2}^{2}}{\gamma_{1}^{2}+\gamma_{2}^{2}} \frac{v_{2}^{(k)}}{v_{1}^{(k)}} & -\gamma_{2} \\
-\frac{\gamma_{1}^{2}}{\gamma_{1}^{2}+\gamma_{2}^{2}} \frac{v_{2}^{(k)}}{v_{1}^{(k)}} & -\frac{\gamma_{1} \gamma_{2}}{\gamma_{1}^{2}+\gamma_{2}^{2}} \frac{v_{2}^{(k)}}{v_{1}^{(k)}} & \gamma_{1} \\
0 & 0 & 0
\end{array}\right) \\
& \mathbf{M}_{\alpha}=\left(\begin{array}{cccc}
0 & \frac{\gamma_{1} M_{1}+\gamma_{2} M_{2}}{\gamma_{1}^{2}+\gamma_{2}^{2}} \frac{v_{2}^{(k)}}{v_{1}^{(k)}} & -M_{2} \\
* & 0 & M_{1} \\
* & * & 0
\end{array}\right)
\end{aligned}
$$

с точностью до замены функций $v_{1,2}^{(1,2)}$, удовлетворяющих $(76)$, на функции $v_{1,2}^{(1,2)}$, удовлетворяющие (108), и замены $\zeta_{k}$ на

$$
\zeta_{k}=\exp \left(\int \frac{d f^{\prime}\left(v_{1}^{(k)}\left(\gamma_{3}^{2}-1\right)-\gamma_{3} v_{2}^{(k)}\right)}{v_{1}^{(k)} f(\mu+d)} \mathrm{d} \gamma_{3}\right) .
$$


Каждый из бивекторов $P_{\alpha}^{(1)}$ и $P_{\alpha}^{(2)}$ обладает четырьмя функциями Казимира

$$
P_{\alpha}^{(k)} \mathrm{d} C=0, \quad P_{\alpha}^{(k)} \mathrm{d} J_{k}=0, \quad P_{\alpha}^{(k)} \mathrm{d} \gamma_{3}=0, \quad P_{\alpha}^{(k)} \mathrm{d}\left(\gamma_{1} M_{2}-\gamma_{2} M_{1}\right)=0
$$

и определяет поле симметрий, действуя на $M_{3}$ :

$$
P_{\alpha}^{(k)} \mathrm{d} M_{3}=X_{S} .
$$

Вторая, или “горизонтальная", составляющая $P_{\beta}$ решений (110) имеет вид

$$
P_{\beta}=\left(\begin{array}{cc}
0 & \boldsymbol{\Gamma}_{\beta} \\
-\boldsymbol{\Gamma}_{\beta}^{\top} & \widetilde{\mathbf{M}}_{\beta}
\end{array}\right), \quad \operatorname{rank} P_{\beta}=2
$$

Здесь $\boldsymbol{\Gamma}_{\beta}-$ та же самая матрица, что и в (87), но

$$
\widetilde{\mathbf{M}}_{\beta}=\left(\begin{array}{ccc}
0 & -M_{3}+\sigma & \frac{d \gamma_{2} f^{\prime}\left(M_{3}\left(\gamma_{3}^{2}-1\right)+\left(\gamma_{1} M_{1}+\gamma_{2} M_{2}\right) \gamma_{3}\right)}{f(\mu+d)} \\
* & 0 & -\frac{d \gamma_{1} f^{\prime}\left(M_{3}\left(\gamma_{3}^{2}-1\right)+\left(\gamma_{1} M_{1}+\gamma_{2} M_{2}\right) \gamma_{3}\right)}{f(\mu+d)} \\
* & * & 0
\end{array}\right),
$$

где

$$
\sigma=\frac{\left(1-\gamma_{3}^{2}\right)\left(\mu+\gamma_{3}^{2} d\right) f^{\prime} M_{3}}{\gamma_{3} f(\mu+d)}+\left(\frac{\left(\mu+\gamma_{3}^{2} d\right) f^{\prime}}{f(\mu+d)}-\frac{\gamma_{3}}{1-\gamma_{3}^{2}}\right)\left(\gamma_{1} M_{1}+\gamma_{2} M_{2}\right) .
$$

Бивектор $P_{\beta}$ обладает следующими функциями Казимира:

$$
P_{\beta} \mathrm{d} C=0, \quad P_{\beta} \mathrm{d} J_{1}=0, \quad P_{\beta} \mathrm{d} J_{2}=0, \quad P_{\beta} \mathrm{d} \frac{\gamma_{2}}{\gamma_{1}}=0 .
$$

Как и для тела вращения на плоскости, бивекторы $P_{\alpha}^{(k)}$ и $P_{\beta}$ имеют формальные особенности при $\gamma_{3}= \pm 1$. Используя свободу выбора коэффициентов $\alpha, \beta$ и свободу выбора линейных интегралов, играющих роль функций Казимира, можно построить решения $P^{(k)}(110)$ без особенностей.

В общем случае на регулярной части пуассонова многообразия бивекторы $P^{(k)}(110)$ имеют по две функции Казимира каждый:

$$
P^{(k)} \mathrm{d} C=0, \quad P^{(k)} \mathrm{d} J_{k}=0, \quad k=1,2 .
$$

Как и ранее, равенство нулю скобок Схоутена эквивалентно существованию линейных интегралов движения $J_{1,2}(108)$ :

$$
\left[P^{(k)}, P^{(k)}\right]=0 \quad \Leftrightarrow \quad \dot{J}_{k}=X\left(J_{k}\right)=0 .
$$

Это означает, что оба эти условия приводят к одной и той же системе уравнений на функции $v_{1,2}^{(k)}(108)$.

Найденная нами пуассонова структура на частично редуцированном фазовом пространстве позволяет изучить структуру векторного поля $X$. 
ПрЕДЛОЖЕНИЕ 7. Для шара на поверхности вращения векторное поле $X$ (104) на шестимерном частично редуцированном фазовом пространстве является суммой гамильтонова поля и поля симметрий $X_{S}(74)$ :

$$
\begin{aligned}
X & =-\frac{\gamma_{3} R}{\beta\left(\gamma_{3} f-\left(1-\gamma_{3}^{2}\right) f^{\prime}\right)} P^{(k)} \mathrm{d} H+\eta_{k} X_{S}, \\
\eta_{k} & =\frac{u_{1}\left(\gamma_{1} M_{1}+\gamma_{2} M_{2}\right)+u_{2} M_{3}}{f(d+\mu)},
\end{aligned}
$$

а коэфбициенты разложения

$$
\begin{aligned}
& u_{1}=-\frac{\gamma_{3} R}{1-\gamma_{3}^{2}}+\frac{\alpha \zeta_{k} \gamma_{3} R f\left(d \gamma_{3}\left(1-\gamma_{3}^{2}\right) v_{1}^{(k)}+\left(\gamma_{3}^{2} d-\mu-d\right) v_{2}^{(k)}\right)}{\beta \mu v_{1}^{(k)}\left(1-\gamma_{3}^{2}\right)\left(\gamma_{3} f-\left(1-\gamma_{3}^{2}\right) f^{\prime}\right)} \\
& u_{2}=R+\frac{\alpha \zeta_{k} \gamma_{3} R f\left(v_{1}^{(k)}\left(\mu+\gamma_{3}^{2} d\right)-d \gamma_{3} v_{2}^{(k)}\right)}{\beta \mu v_{1}^{(k)}\left(\gamma_{3} f-\left(1-\gamma_{3}^{2}\right) f^{\prime}\right)}
\end{aligned}
$$

зависят от функций $v_{1}^{(k)}$ и $v_{2}^{(k)}$, входящих в определение(107) интегралов движения $J_{k}$.

Если положить $\alpha=$ const и

$$
\beta=-\frac{\left(1-\gamma_{3}^{2}\right)\left(\gamma_{3} v_{1}^{(k)}-v_{2}^{(k)}\right)^{2} d+\mu\left(\left(v_{1}^{(k)}\right)^{2}+\left(1-\gamma_{3}^{2}\right)\left(v_{2}^{(k)}\right)^{2}\right)}{\left(\gamma_{3} f-\left(1-\gamma_{3}^{2}\right) f^{\prime}\right)\left(v_{1}^{(k)}\left(1-\gamma_{3}^{2}\right)+\gamma_{3} v_{2}^{(k)}\right)} \frac{\gamma_{3} f \alpha \zeta_{k}}{\mu v_{1}^{(k)}}
$$

мы получим

$$
X=-\frac{\gamma_{3} R}{\beta\left(\gamma_{3} f-\left(1-\gamma_{3}^{2}\right) f^{\prime}\right)} P^{(k)} \mathrm{d} H+\delta J_{k} X_{S}
$$

где

$$
\delta=\frac{R\left(\gamma_{3} v_{1}^{(k)}-v_{2}^{(k)}\right)}{\left(1-\gamma_{3}^{2}\right)\left(\gamma_{3} v_{1}^{(k)}-v_{2}^{(k)}\right)^{2} d+\mu\left(\left(v_{1}^{(k)}\right)^{2}+\left(1-\gamma_{3}^{2}\right)\left(v_{2}^{(k)}\right)^{2}\right)} .
$$

ПреДЛОЖЕНИЕ 8. Для шара на поверхности вращения векторное поле $X$ (104) конформно-гамильтоново,

$$
X=-\frac{\gamma_{3} R}{\beta\left(\gamma_{3} f-\left(1-\gamma_{3}^{2}\right) f^{\prime}\right)} P^{(k)} \mathrm{d} H,
$$

относительно бивектора Пуассона четвертого ранга при

$$
J_{k}=0, \quad k=1,2 .
$$

6.2. Гамильтонизация на редуцированном подмногообразии. Следуя работе [27], рассмотрим инвариантное относительно действия (72) группы симметрий $\mathrm{SO}(2)$ подпространство с координатами

$$
\gamma_{3}, \quad K_{1}=f(\gamma, M), \quad K_{2}=\mu \omega_{3}=\frac{\mu M_{2}+d \gamma_{3}(\gamma, M)}{\mu+d}, \quad K_{3}=\frac{\gamma_{2} M_{1}-\gamma_{2} M_{1}}{\sqrt{1-\gamma_{3}^{2}}} .
$$

Как и ранее, эти координаты определены только при $\gamma_{3} \neq \pm 1$. 
Бивекторы Пуассона $P^{(1,2)}(110)$ имеют одну общую проекцию на это редуцированное четырехмерное пространство. Если положить

$$
\beta=-\frac{R}{f-\left(\left(1-\gamma_{3}^{2}\right) / \gamma_{3}\right) f^{\prime}} \quad \text { и } \quad \varkappa=\frac{\sqrt{1-\gamma_{3}^{2}} R}{f-\left(\left(1-\gamma_{3}^{2}\right) / \gamma_{3}\right) f^{\prime}},
$$

то эта проекция имеет вид

$$
\widehat{P}=\varkappa\left(\begin{array}{cccc}
0 & 0 & 0 & 1 \\
0 & 0 & 0 & \frac{f^{\prime}}{\gamma_{3}} K_{2} \\
0 & 0 & 0 & \frac{d}{(\mu+d) f} K_{1} \\
* & * & * & 0
\end{array}\right), \quad \operatorname{rank} \widehat{P}=2 .
$$

Исходное векторное поле $X$ (114) на редуцированном подмногообразии является гамильтоновым,

$$
\widehat{X}=\widehat{P} \mathrm{~d} H,
$$

по отношению к данной пуассоновой структуре второго ранга, обладающей функциями Казимира $J_{1,2}$. Как и ранее, поделив два гамильтоновых уравнения

$$
\dot{K}_{1}=\left\{H, K_{1}\right\}=-\frac{\varkappa f^{\prime}}{\gamma_{3}(\mu+d)} K_{2} K_{3}, \quad \dot{K}_{2}=\left\{H, K_{2}\right\}=-\frac{\varkappa d}{f(\mu+d)^{2}} K_{1} K_{3}
$$

на третье

$$
\dot{\gamma}_{3}=\left\{H, g_{3}\right\}=-\frac{\varkappa K_{3}}{\mu+d},
$$

мы получим систему линейных неавтономных дифференциальных уравнений первого порядка

$$
\frac{\mathrm{d} K_{1}}{\mathrm{~d} \gamma_{3}}=\frac{f^{\prime}}{\gamma_{3}} K_{2}, \quad \frac{\mathrm{d} K_{2}}{\mathrm{~d} \gamma_{3}}=\frac{d}{f(\mu+d)} K_{1},
$$

аналог которых был получен Раусом [75] в переменных, связанных с полуподвижной системой координат.

6.3. Приведение скобок Пуассона к каноническим. Рассмотрим приведение бивекторов $P^{(k)}(110)$ к канонической форме, используя отображения Пуассона

$$
\psi_{k}:(\gamma, M) \rightarrow(\gamma, L),
$$

которые по форме совпадают с отображениями (91), но в которых

$$
\varrho_{k}=\int \frac{v_{1}^{(k)}}{\left(v_{2}^{(k)}\right)^{2} \zeta_{k}}\left(1-\frac{\left(1-\gamma_{3}^{2}\right)\left(\mu+\gamma_{3}^{2} d\right) f^{\prime}}{\gamma_{3} f(\mu+d)}\right) \mathrm{d} \gamma_{3},
$$

функции $\zeta_{k}$ определены соотношением (112), а функции $v_{1,2}^{(1,2)}$ удовлетворяют уравнениям (108). Исправленные таким образом отображения $\psi_{k}$ приводят бивекторы Пуассона (110) к каноническому бивектору (10) на алгебре Ли $e^{*}(3)$ при $\alpha=$ const. 
Используя это отображение, мы можем отождествить исходную динамическую систему (104) с динамической системой на сфере с каноническими скобками Пуассона. Соответствующая функция Гамильтона будет иметь стандартный вид

$$
H=A L_{3}^{2}+B\left(L_{1} \gamma_{2}-L_{2} \gamma_{1}\right)^{2}+b C L_{3}+b^{2} D,
$$

где $A, B, C$ и $D$ - функции только от $\gamma_{3}$, а второй интеграл движения будет линейным по $L_{3}$.

Так как функции $\beta$ произвольны, то мы всегда можем отождествить коэффициенты $B$ в гамильтонианах (93) и (120), отвечающих разным неголономным системам. Однако в общем случае вопрос об отождествлении гамильтониана (120), отвечающего шару на какой-либо конкретной поверхности вращения, с гамильтонианом (93), отвечающим некоему телу вращения специального вида на плоскости, с точностью до канонических преобразований координат на сфере остается открытым.

6.4. Движение по параболоиду вращения. Предположим, что центр шара движется по параболоиду вращения $z=c\left(x^{2}+y^{2}\right)$. В этом случае

$$
f\left(\gamma_{3}\right)=-\frac{1}{2 c \gamma_{3}}
$$

и уравнения (108) имеют следующие решения:

$$
v_{1}^{(1,2)}=\gamma_{3}^{ \pm \nu}, \quad v_{2}^{(1,2)}=\gamma_{3}^{1 \pm \nu} \pm \frac{\nu}{1 \pm \nu} \gamma_{3}^{ \pm \nu-1}
$$

где $\nu$ - вещественное число, определяемое массой шара, его радиусом и величиной тензора инерции:

$$
\nu=\sqrt{\frac{\mu}{\mu+d}}, \quad d=m R^{2} .
$$

ЗАмЕчАниЕ 14. Согласно [27], произведение этих интегралов движения $H_{2}=J_{1} J_{2}$ будет алгебраическим интегралом движения второго порядка по моментам.

В этом случае в определение бивектора Пуассона и отображения, приводящего его к канонической форме, входят функции

$$
\zeta_{1,2}=\gamma_{3}^{1 \pm \nu} \quad \text { и } \quad \varrho_{1,2}= \pm \frac{(1 \mp \nu)^{2}}{2 \nu} \frac{\gamma_{3}^{2} \mp \nu\left(1-\gamma_{3}^{2}\right)}{\gamma_{3}^{2} \pm \nu\left(1-\gamma_{3}^{2}\right)} \gamma_{3}^{\mp 2 \nu} .
$$

Используя, например, вместо интегралов движения $J_{1,2}$ их линейные комбинации

$$
J_{ \pm}=\frac{\nu+1}{2 \nu} J_{1} \pm \frac{\nu-1}{2 n u} J_{2} \equiv v_{1}^{( \pm)}\left(\gamma_{1} M_{1}+\gamma_{2} M_{2}\right)+v_{2}^{( \pm)} M_{3},
$$

мы получим бивекторы $P_{\alpha}^{( \pm)}(111)$, которые не имеют особенностей при $\gamma_{1,2}=0$ и $\gamma_{3}= \pm 1$. Тем самым, используя свободу выбора линейных интегралов движения, можно получить пуассоновы структуры без особенностей в точках пуассонова многообразия, отвечающих устойчивым положениям равновесия. 
Теперь рассмотрим отображение Пуассона (91), приводящее скобки к канонической форме. Если положить $c=0$ в $(91)$, то, применяя пуассоново отображение к бивектору $P^{(1)}$, обладающему функцией Казимира $J_{1}=b$, мы получим следующие выражения для интегралов движения на сфере:

$$
\begin{gathered}
H=\frac{1-\nu^{2}}{2 d\left(1-\gamma_{3}^{2}\right)}\left(\frac{\alpha^{2} \gamma_{3}^{2 \nu} L_{3}^{2}}{(1-\nu)^{2}}+\beta^{2}\left(\gamma_{2} L_{1}-\gamma_{1} L_{2}\right)^{2}\right. \\
\left.+\frac{\alpha\left(2 \gamma_{3}^{2}-1\right) b L_{3}}{\nu}+\frac{(1-\nu)^{2} b^{2} \gamma_{3}^{-2 \nu}}{4 \nu^{2}}\right)
\end{gathered}
$$

и

$$
J_{2}=\frac{2 \alpha \nu}{\nu^{2}-1} L_{3}
$$

Используя стандартные сферические координаты (100), мы, как и ранее, можем проинтегрировать уравнения движения в квадратурах совершенно стандартным способом.

\section{7. Система Суслова}

Рассмотрим движение твердого тела с неподвижной точкой, подчиненного неголономной связи

$$
(\omega, a)=0
$$

где $\omega$ - вектор угловой скорости и $a$ - произвольный постоянный в теле вектор. Реализация подобной связи, предложенной Г. К. Сусловым, обсуждается в работах [18], [77].

Уравнения движения в подвижной системе координат, жестко связанной с телом, имеют вид

$$
\frac{\mathrm{d}}{\mathrm{d} t} \mathbf{I} \omega=\mathbf{I} \omega \times \omega+\lambda a, \quad \frac{\mathrm{d}}{\mathrm{d} t} \gamma=\gamma \times \omega
$$

где $\mathbf{I}$ - тензор инерции, $\lambda$ - неопределенный множитель Лагранжа, $\gamma$ - вектор Пуассона, отвечающий неподвижному орту.

Связанную с телом подвижную систему координат можно всегда выбрать так, чтобы $a=(0,0,1)$ и соответствующий центробежный момент инерции равнялся нулю: $I_{12}=0$. В этом случае уравнение связи принимает вид

$$
\omega_{3}=0,
$$

а уравнения движения записываются в форме

$$
\begin{gathered}
I_{11} \dot{\omega}_{1}=-\omega_{2}\left(I_{13} \omega_{1}+I_{23} \omega_{2}\right), \quad I_{22} \dot{\omega}_{2}=\omega_{1}\left(I_{13} \omega_{1}+I_{23} \omega_{2}\right), \quad \dot{\omega}_{3}=0, \\
\dot{\gamma}_{1}=-\gamma_{3} \omega_{2}, \quad \dot{\gamma}_{2}=\gamma_{3} \omega_{1}, \quad \dot{\gamma}_{3}=\gamma_{1} \omega_{2}-\gamma_{2} \omega_{1} .
\end{gathered}
$$

Соответствующее векторное поле $X$ обладает интегралами движения

$$
H_{1}=\frac{1}{2}\left(I_{11} \omega_{1}^{2}+I_{22} \omega_{2}^{2}\right), \quad H_{2}=(\gamma, \gamma), \quad H_{3}=\omega_{3}
$$

И

$$
H_{4}=(f, \gamma), \quad f=\left(f_{1}(\omega), f_{2}(\omega), f_{3}(\omega)\right)
$$


Входящий в это определение вектор $f$ является некоторым частным решением уравнения $\dot{f}+\omega \times f=0$, так что

$$
\dot{H}_{4}=(\dot{f}, \gamma)+(f, \dot{\gamma})=(\dot{f}, \gamma)+(f, \gamma \times \omega)=(\dot{f}+\omega \times f, \gamma)=0
$$

Кроме этих четырех интегралов движения векторное поле $X$ (123) обладает инвариантной мерой

$$
\mu=g^{-1} \mathrm{~d} \gamma \mathrm{d} \omega, \quad g=I_{13} \omega_{1}+I_{23} \omega_{2} .
$$

Свойства этой меры, а также условия существования однозначного интеграла движения $H_{4}$ подробно обсуждаются в работе [18].

Как и ранее, мы подставим выражения для интегралов движения $H_{1}, \ldots, H_{4}$ и линейный по $\omega$ анзац

$$
P_{i j}=\sum_{k=1}^{3} c_{i j}^{k}(\gamma) \omega_{k}+d_{i j}(\gamma)
$$

где $c_{i j}^{k}$ и $d_{i j}$ - неизвестные функции от $\gamma$, в уравнения (12) и будем решать полученные таким образом уравнения. Однако, в отличие от предыдущих случаев, мы выделим один из интегралов $H_{3}=\omega_{3}$ и будем предполагать, что именно этот интеграл является функцией Казимира искомого бивектора Пуассона:

$$
P \mathrm{~d} H_{3}=P \mathrm{~d} \omega_{3}=0 .
$$

Тем самым, уравнение связи (122) фиксирует симплектический лист, на котором эволюционирует рассматриваемая динамическая система.

7.1. Пуассоновы структуры при движении по инерции. Уравнения (12) для бивектора Пуассона имеют несколько решений второго ранга. Мы выпишем только одно из них:

$$
P=\left(\begin{array}{cc}
0 & \widehat{\mathbf{W}} \\
-\widehat{\mathbf{W}}^{\top} & 0
\end{array}\right), \quad \widehat{\mathbf{W}}=\left(\begin{array}{ccc}
-g_{1} I_{22} \omega_{2} & g_{1} I_{11} \omega_{1} & 0 \\
-g_{2} I_{22} \omega_{2} & g_{2} I_{11} \omega_{1} & 0 \\
-g_{3} I_{22} \omega_{2} & g_{3} I_{11} \omega_{1} & 0
\end{array}\right)
$$

где $g_{k}$ - произвольные функции от $\gamma$, связанные соотношением

$$
g_{1} \gamma_{1}+g_{2} \gamma_{2}+g_{3} \gamma_{3}=0
$$

Уравнения (12) имеют также решения четвертого ранга. Мы выпишем одно из таких решений в случае, когда дополнительный интеграл $H_{4}(125)$ является линейным полиномом по скоростям $\omega$, так же как и используемый нами анзац для бивектора Пуассона. Напомним [18], что при

$$
I_{13}=0, \quad I_{23}=\sqrt{\left(I_{11}-I_{22}\right) I_{22}}
$$

интеграл движения $H_{4}(125)$ имеет следующий вид:

$$
H_{4}=(\mathbf{I} \gamma, \omega)=I_{11} \omega_{1} \gamma_{1}+I_{22} \omega_{2} \gamma_{2}+I_{23} \omega_{2} \gamma_{3} .
$$


В этом случае решение уравнений (12),

$$
P=\alpha P_{\alpha}+\beta P_{\beta},
$$

состоит из двух совместных бивекторов Пуассона ранга два: $P_{\alpha}$ и $P_{\beta}$. Первый бивектор имеет вид

$$
P_{\alpha}=\left(\begin{array}{cc}
\mathbf{W}_{\alpha} & 0 \\
0 & 0
\end{array}\right)
$$

где

$$
\mathbf{W}_{\alpha}=\frac{\left(I_{23} \gamma_{3}+I_{22} \gamma_{2}\right)^{2}}{I_{23}\left(I_{23} \gamma_{2}-I_{22} \gamma_{3}\right)}\left(\begin{array}{ccc}
0 & I_{23} \omega_{2} & -I_{22} \omega_{2} \\
-I_{23} \omega_{2} & 0 & I_{11} \omega_{1} \\
I_{22} \omega_{2} & -I_{11} \omega_{1} & 0
\end{array}\right)
$$

Второй бивектор равен

$$
P_{\beta}=\left(\begin{array}{cc}
0 & \boldsymbol{\Gamma}_{\beta} \\
-\boldsymbol{\Gamma}_{\beta}^{\top} & \mathbf{W}_{\beta}
\end{array}\right)
$$

где

$\boldsymbol{\Gamma}_{\beta}=\left(\begin{array}{ccc}\left(I_{23} \gamma_{3}+I_{22} \gamma_{2}\right) \gamma_{1} & -I_{11} \gamma_{1}^{2} & 0 \\ -\frac{\left(I_{23} \gamma_{3}+I_{22} \gamma_{2}\right)\left(I_{23}\left(\gamma_{1}^{2}+\gamma_{3}^{2}\right)+I_{22} \gamma_{2} \gamma_{3}\right)}{I_{23} \gamma_{2}-I_{22} \gamma_{3}} & \frac{\left(I_{23}\left(\gamma_{1}^{2}+\gamma_{3}^{2}\right)+I_{22} \gamma_{2} \gamma_{3}\right) I_{11} \gamma_{1}}{I_{23} \gamma_{2}-I_{22} \gamma_{3}} & 0 \\ \frac{\left(I_{23} \gamma_{3}+I_{22} \gamma_{2}\right)\left(I_{23} \gamma_{2} \gamma_{3}+I_{22}\left(\gamma_{1}^{2}+\gamma_{2}^{2}\right)\right)}{I_{23} \gamma_{2}-I_{22} \gamma_{3}} & -\frac{\left(I_{23} \gamma_{2} \gamma_{3}+I_{22}\left(\gamma_{1}^{2}+\gamma_{2}^{2}\right)\right) I_{11} \gamma_{1}}{I_{23} \gamma_{2}-I_{22} \gamma_{3}} & 0\end{array}\right)$

и

$$
\mathbf{W}_{\beta}=\left(\begin{array}{ccc}
0 & H_{4} & 0 \\
-H_{4} & 0 & 0 \\
0 & 0 & 0
\end{array}\right)
$$

Коэффициенты $\alpha, \beta$ в разложении (126) являются произвольными функциями от функций Казимира бивекторов $P_{\beta}$ и $P_{\alpha}$ соответственно. Например, бивектор $P_{\alpha}$ обладает следующими функциями Казимира:

$$
P_{\alpha} \mathrm{d} H_{4}=0, \quad P_{\alpha} \mathrm{d} \omega_{i}=0, \quad i=1,2,3,
$$

и поэтому коэффициент $\beta$ является произвольной функцией от $\omega$. Для бивектора $P_{\beta}$ нам удалось найти всего три функции Казимира из четырех:

$$
P_{\beta} \mathrm{d} H_{4}=0, \quad P_{\beta} \mathrm{d} H_{2}=0, \quad P_{\beta} \mathrm{d} \omega_{3}=0 .
$$

Полное решение (126) является в общем случае бивектором Пуассона ранга четыре с функциями Казимира

$$
P \mathrm{~d} H_{4}=0, \quad P \mathrm{~d} \omega_{3}=0 .
$$

Если подставить в определение бивектора $P(126)$ явные мероморфные решения уравнений Пуассона, приведенные для этого случая в работе [40], то можно доказать, что особенность при $I_{23} \gamma_{2}-I_{22} \gamma_{3}=0$ при физическом движении никогда не достигается. 
ПРЕДЛОЖЕНИЕ 9. Векторное поле X (123) на регулярной части пуассонова многообразия можно представить в виде

$$
X=\nu_{1} P \mathrm{~d} H_{1}+\nu_{2} P \mathrm{~d} H_{2},
$$

где

$u$

$$
\nu_{1}=-\frac{\omega_{2}\left(I_{23}\left(\omega_{1} \gamma_{2}-\omega_{2} \gamma_{1}\right)+\left(I_{11}-I_{22}\right) \omega_{1} \gamma_{3}\right)}{\beta I_{11} H_{4}\left(I_{22}\left(\omega_{1} \gamma_{2}-\omega_{2} \gamma_{1}\right)+I_{23} \omega_{1} \gamma_{3}\right)}
$$

$$
\nu_{2}=\frac{1}{2 \alpha} \frac{I_{23}\left(I_{23}\left(\omega_{1} \gamma_{1} \gamma_{2}-\omega_{2} \gamma_{1}^{2}-\omega_{2} \gamma_{3}^{2}\right)-\left(\omega_{1} \gamma_{1}+\omega_{2} \gamma_{2}\right) I_{22} \gamma_{3}\right)}{H_{4}\left(I_{23} \gamma_{3}+I_{22} \gamma_{2}\right)^{2}} .
$$

Обсуждение возможных приложений этого разложения исходного векторного поля по гамильтоновым выходит за рамки данного обзора.

Итак, в этом частном случае мы доказали, что и для задачи Суслова совместные поверхности интегралов движения могут быть отождествлены с лагранжевыми подмногообразиями.

7.2. Частный случай движения в потенциальном поле. Если вектор $a=(0,0,1)$ является собственным вектором тензора инерции: $\mathbf{I} a=\mu a, \mu=I_{33}$, т. е. если

$$
I_{13}=I_{23}=0,
$$

то можно рассмотреть интегрируемую систему, описывающую движение в потенциальном поле [18]. В этом частном случае уравнения движения имеют вид

$$
\begin{gathered}
I_{11} \dot{\omega}_{1}=\gamma_{2} \frac{\partial U}{\partial \gamma_{3}}-\gamma_{3} \frac{\partial U}{\partial g_{2}}, \quad I_{22} \dot{\omega}_{2}=\gamma_{3} \frac{\partial U}{\partial \gamma_{1}}-\gamma_{1} \frac{\partial U}{\partial g_{3}}, \quad \omega_{3}=0 \\
\dot{\gamma}_{1}=-\gamma_{3} \omega_{2}, \quad \dot{\gamma}_{2}=\gamma_{3} \omega_{1}, \quad \dot{\gamma}_{3}=\gamma_{1} \omega_{2}-\gamma_{2} \omega_{1} .
\end{gathered}
$$

Согласно [18] соответствующее векторное поле $X$ гамильтоново:

$$
X=P \mathrm{~d} H, \quad H=\frac{1}{2}(\gamma, \gamma),
$$

если

$$
U(\gamma)=\mu \gamma_{3}+V\left(\gamma_{1}, \gamma_{2}\right)
$$

Бивектор Пуассона в этом случае равен

$$
P=\left(\begin{array}{cccccc}
0 & 0 & -\omega_{2} & 0 & I_{22}^{-1} \partial_{3} U & 0 \\
0 & 0 & \omega_{1} & -I_{11}^{-1} \partial_{3} U & 0 & 0 \\
\omega_{2} & -\omega_{1} & 0 & I_{11}^{-1} \partial_{2} U & -I_{22}^{-1} \partial_{1} U & 0 \\
0 & I_{11}^{-1} \partial_{3} U & -I_{11}^{-1} \partial_{2} U & 0 & 0 & 0 \\
-I_{22}^{-1} \partial_{3} U & 0 & I_{22}^{-1} \partial_{1} U & 0 & 0 & 0 \\
0 & 0 & 0 & 0 & 0 & 0
\end{array}\right),
$$

где $\partial_{k}=\partial / \partial \gamma_{k}$. Если $\mu \neq 0$, то это пуассонова структура четвертого ранга:

$$
\operatorname{rank} P=4 .
$$

Как и ранее, это означает, что совместные поверхности интегралов движения для этой неголономной системы могут быть отождествлены с лагранжевыми подмногообразиями. 


\section{8. Заключение}

Изучение неголономного движения твердого тела обычно начинается с рассмотрения свободного движения твердого тела, затем накладываются голономные и неголономные связи и делается редукция по симметриям, существующим в задаче. Напомним, что фазовым пространством для свободного движения твердого тела является 12-мерное пространство $T E(3)$, где $E(3)$ - группа движений трехмерного евклидова пространства. Данное пространство является пуассоновым многообразием, снабженным каноническим бивектором Пуассона, что и позволяет нам переписать уравнения движения свободного твердого тела в гамильтоновой форме.

На каждой стадии перехода от гамильтоновых уравнений движения свободного тела к уравнениям, описывающим неголономное движение на редуцированном по симметриям фазовом пространстве, можно найти следы (нелинейные деформации) исходной пуассоновой структуры на многообразии $T E(3)$. Детальное изучение всех проявлений исходной пуассоновой структуры еще впереди, а в этом обзоре мы собрали все основные известные на данное время сведения о нелинейных пуассоновых структурах, возникающих в неголономной механике в трехмерном евклидовом пространстве.

Авторы выражают благодарность А. В. Болсинову, И. А. Бизяеву, А. А. Килину за полезные обсуждения и комментарии, а также В. В. Козлову за полезные советы, способствовавшие существенному улучшению работы.

\section{Список литературы}

[1] А.А. Аграчев, Ю.Л. Сачков, Геометрическая теория управления, Физматлит, M., 2005, 392 с.; пер. с англ.: А. A. Agrachev, Yu.L. Sachkov, Control theory from the geometric viewpoint, Encyclopaedia Math. Sci., 87, Control theory and optimization, II, Springer-Verlag, Berlin, 2004, xiv+412 pp.

[2] P. Appell, Traité de mécanique rationelle, v. II: Dynamique des systèmes. Mécanique analytique, Gauthier-Villars, Paris, 1896, iv+538 pp.

[3] P. Appell, "Sur l'intégration des équations du mouvement d'un corps pesant de révolution roulant par une arête circulaire sur un plan horizontal; cas particulier du cerceau", Rend. Circ. Mat. Palermo, 14:1 (1900), 1-6.

[4] V.I. Arnold, "Symplectic geometry and topology", J. Math. Phys., 41:6 (2000), 3307-3343.

[5] В. И. Арнольд, В. В. Козлов, А. И. Нейштадт, Математические аспекты классической и небесной механики, Эдиториал УРСС, М., 2002, 416 с.; Динамические системы - 3, Итоги науки и техники. Сер. Соврем. пробл. матем. Фундам. напр., 3, ВИНИТИ, М., 1985, 5-290; англ. пер.: V. I. Arnol'd, V. V. Kozlov, A. I. Nersshtadt, "Mathematical aspects of classical and celestial mechanics", Dynamical systems, III, Encyclopaedia Math. Sci., 3, Springer-Verlag, Berlin, 1988, 1-291.

[6] P. Balseiro, L. C. García-Naranjo, "Gauge transformations, twisted Poisson brackets and Hamiltonization of nonholonomic systems", Arch. Ration. Mech. Anal., 205:1 (2012), 267-310.

[7] L. Bates, J. Śniatycki, "Nonholonomic reduction", Rep. Math. Phys., 32:1 (1993), 99-115.

[8] M. Batista, "The nearly horizontally rolling of a thick disk on a rough plane", Regul. Chaotic Dyn., 13:4 (2008), 344-354.

[9] I. A. Bizayev, A. V. Tsiganov, "On the Routh sphere problem", J. Phys. A, 46:8 (2013), 085202, 11 pp. 
[10] A. M. Bloch, J. E. Marsden, D. V. Zenkov, "Nonholonomic dynamics", Notices Amer. Math. Soc., 52:3 (2005), 324-333.

[11] S. V. Bolotin, T. V. Popova, "On the motion of a mechanical system inside a rolling ball", Regul. Chaotic Dyn., 18:1-2 (2013), 159-165.

[12] A. V. Bolsinov, A. V. Borisov, I. S. Mamaev, "Hamiltonization of nonholonomic systems in the neighborhood of invariant manifolds", Regul. Chaotic Dyn., 16:5 (2011), 443-464.

[13] А. В. Болсинов, А. В. Борисов, И. С. Мамаев, "Геометризация теоремы Чаплыгина о приводящем множителе", Нелинейная динам., 9:4 (2013), 627-640.

[14] А. В. Болсинов, А. А. Килин, А. О. Казаков, "Топологическая монодромия в неголономных системах", Нелинейная динам., 9:2 (2013), 203-227.

[15] A. V. Bolsinov, A. A. Oshemkov, "Bi-Hamiltonian structures and singularities of integrable systems", Regul. Chaotic Dyn., 14:4-5 (2009), 431-454.

[16] А. В. Борисов, Ю.Н.Федоров, "О двух видоизмененных интегрируемых задачах динамики", Вестн. Моск. ун-та. Сер. 1. Матем., мех., 1995, № 6, 102-105; Неголономные динамические системы. Интегрируемость, хаос, странные аттракторы, ред. А. В. Борисов, И. С. Мамаев, Институт компьютерных исследований, М.-Ижевск, 2002, 67-70; англ. пер.: А. V. Borisov, Y. N. Fedorov, "On two modified integrable problems in dynamics", Moscow Univ. Math. Bull., 50:6 (1995), 16-18.

[17] A. V. Borisov, Y. N. Fedorov, I.S. Mamaev, "Chaplygin ball over a fixed sphere: an explicit integration", Regul. Chaotic Dyn., 13:6 (2008), 557-571.

[18] A. V. Borisov, A. A. Kilin, I. S. Mamaev, "Hamiltonicity and integrability of the Suslov problem", Regul. Chaotic Dyn., 16:1-2 (2011), 104-116.

[19] A. V. Borisov, A. A. Kilin, I. S. Mamaev, "On the model of non-holonomic billiard", Regul. Chaotic Dyn., 16:6 (2011), 653-662.

[20] А. В. Борисов, А.А. Килин, И. С. Мамаев, "Обобщение преобразования Чаплыгина и явное интегрирование шарового подвеса", Нелинейная динам., 7:2 (2011), 313-338.

[21] А. В. Борисов, И. С. Мамаев, Пуассоновы структуры и алгебры Ли в гамилътоновой механике, Библиотека "R \& C Dynamics", Издательский дом "Удмуртский университет", Ижевск, 1999, 464 с.

[22] А. В. Борисов, И. С. Мамаев, "Гамильтоновость задачи Чаплыгина о качении шара", Матем. заметки, 70:5 (2001), 793-795; англ. пер.: А. V. Borisov, I. S. Mamaev, "Chaplygin's ball rolling problem is Hamiltonian", Math. Notes, 70:5 (2001), $720-723$.

[23] A. V. Borisov, I. S. Mamaev, "Rolling of a rigid body on plane and sphere. Hierarchy of dynamics", Regul. Chaotic Dyn., 7:2 (2002), 177-200.

[24] A. V. Borisov, I. S. Mamaev, "Conservation laws, hierarchy of dynamics and explicit integration of nonholonomic systems", Regul. Chaotic Dyn., 13:5 (2008), 443-490.

[25] А. В. Борисов, И. С. Мамаев, “Динамика саней Чаплыгина”, ПмМ, 73:2 (2009), 219-225; англ. пер.: А. V. Borisov, I. S. Mamaev, "The dynamics of a Chaplygin sleigh", J. Appl. Math. Mech., 73:2 (2009), 156-161.

[26] A. V. Borisov, I. S. Mamaev, I. A. Bizyaev, "The hierarchy of dynamics of a rigid body rolling without slipping and spinning on a plane and a sphere", Regul. Chaotic Dyn., 18:3 (2013), 277-328.

[27] A. V. Borisov, I. S. Mamaev, A. A. Kilin, "Rolling of a ball on a surface. New integrals and hierarchy of dynamics", Regul. Chaotic Dyn., 7:2 (2002), 201-219.

[28] F. Cantrijn, J. Cortés, M. de León, D. Martín de Diego, "On the geometry of generalized Chaplygin systems", Math. Proc. Cambridge Philos. Soc., 132:2 (2002), 323-351.

[29] С.А. Чаплыгинъ, "О нькоторомъ возможномъ обобщеніи теоремы площадей съ примъненіемъ къ задачь о катаніи шаровъ”, Матем. сб., 20:1 (1897), 1-32.

[30] С. А. Чаплыгин, "О движении тяжелого тела вращения на горизонтальной плоскости", Тр. отд. физ. наук Моск. об-ва любителей естествознания, антропологии и этнографии, 9:1 (1897), 10-16; англ. пер.: S. A. Chaplygin, "On a motion of 
a heavy body of revolution on a horizontal plane", Regul. Chaotic Dyn., 7:2 (2002), $119-130$.

[31] С. А. Чаплыгинъ, "О катаніи шара по горизонтальной плоскости", Матем. сб., 24:1 (1903), 139-168; англ. пер.: S. A. Chaplygin, "On a ball's rolling on a horizontal plane", Regul. Chaotic Dyn., 7:2 (2002), 131-148.

[32] С. А. Чаплыгинъ, "Къ теоріи движенія неголономныхъ системъ. Теорема о приводящемъ множитель", Матем. сб., 28:2 (1912), 303-314; англ. пер.: S. А. Chaplygin, "On the theory of motion of nonholonomic systems. The reducing-multiplier theorem", Regul. Chaotic Dyn., 13:4 (2008), 369-376.

[33] R. Cushman, "Routh's sphere", Rep. Math. Phys., 42:1-2 (1998), 47-70.

[34] R. Cushman, J. J. Duistermaat, "Non-Hamiltonian monodromy", J. Differential Equations, 172:1 (2001), 42-58.

[35] R. Cushman, H. Duistermaat, J. Śniatycki, Geometry of nonholonomically constrained systems, Adv. Ser. Nonlinear Dynam., 26, World Scientific Publishing Co., Hackensack, NJ, 2010, xviii+404 pp.

[36] K. Ehlers, J. Koiller, R. Montgomery, P. M. Rios, "Nonholonomic systems via moving frames: Cartan equivalence and Chaplygin Hamiltonization", The breadth of symplectic and Poisson geometry, Progr. Math., 232, Birkhäuser Boston, Boston, MA, 2005, $75-120$.

[37] F. Fassò, A. Giacobbe, N. Sansonetto, "Periodic flows, rank-two Poisson structures, and nonholonomic mechanics", Regul. Chaotic Dyn., 10:3 (2005), 267-284.

[38] Ю.Н. Федоров, "О двух интегрируемых неголономных системах в классической динамике", Вестн. Моск. ун-та. Сер. 1. Матем., мех., 1989, № 4, 38-41; англ. пер.: Yu. N. Fedorov, "Two integrable nonholonomic systems in classical dynamics", Moscow Univ. Math. Bull., 44:4 (1989), 7-12.

[39] Y.N. Fedorov, B. Jovanović, "Hamiltonization of the generalized Veselova LR system", Regul. Chaotic Dyn., 14:4-5 (2009), 495-505.

[40] Y. N. Fedorov, A. J. Maciejewski, M. Przybylska, "The Poisson equations in the nonholonomic Suslov problem: integrability, meromorphic and hypergeometric solutions", Nonlinearity, 22:9 (2009), 2231-2260.

[41] O. E. Fernandez, T. Mestdag, A. M. Bloch, "A generalization of Chaplygin's reducibility theorem", Regul. Chaotic Dyn., 14:6 (2009), 635-655.

[42] E. G. Gallop, "On the rise of a spinning top", Part 3, Proc. Cambridge Philos. Soc., 19 (1904), 356-373.

[43] П.А. Гриффитс, Внешние дифференциальные системь и вариационное исчисление, Мир, М., 1986, 360 с.; пер. с англ.: Ph.A. Griffiths, Exterior differential systems and the calculus of variations, Progr. Math., 25, Birkhäuser, Boston, MA, 1983, ix+335 pp.

[44] J. Hermans, "A symmetric sphere rolling on a surface", Nonlinearity, 8:4 (1995), 493-515.

[45] S. Hochgerner, L. García-Naranjo, "G-Chaplygin systems with internal symmetries, truncation, and an (almost) symplectic view of Chaplygin's ball", J. Geom. Mech., 1:1 (2009), 35-53.

[46] A. Ibort, M. de León, J. C. Marrero, D. Martín de Diego, "Dirac brackets in constrained dynamics", Fortschr. Phys., 47:5 (1999), 459-492.

[47] J.H. Jellet, A treatise on the theory of friction, MacMillan and Co., Dublin, Hodges, Foster, and Co., 1872, 220 pp.

[48] J. Koiller, "Reduction of some classical non-holonomic systems with symmetry", Arch. Ration. Mech. Anal., 118:2 (1992), 113-148.

[49] А.Н. Колмогоров, "О динамических системах с интегральным инвариантом на торе", Докл. АН СCCP, 93:5 (1953), 763-766; англ. пер.: A. N. Kolmogorov, "On dynamical systems with an integral invariant on a torus", Selected works of A. N. Kolmogorov, v. I: Mathematics and mechanics, Mathematics and Its Applications, Soviet Ser., 25, ed. V. M. Tikhomirov, Kluwer Acad. Publ., Dordrecht, 1991, 344-348. 
[50] D. Korteweg, "Ueber eine ziemlich verbreitete unrichtige Behandlungsweise eines Problems der rollenden Bewegung, über die Theorie dieser Bewegung und insbesondere über kleine rollende Schwingungen um eine Gleichgewichtslage", Nieuw Archiefvoor Wiskunde, 4 (1899), 130-155.

[51] В. В. Козлов, “Динамика систем с неинтегрируемыми связями. I", Вестн. Моск. ун-та. Сер. 1. Матем., мех., 1982, № 3, 92-100; II, 1982, № 4, 70-76; III, 1983, № 3, 102-111; IV, 1987, № 5, 76-83; V, 1988, № 6, 51-54; англ. пер.: V. V. Kozlov, "The dynamics of systems with nonintegrable constraints. I", Mosc. Univ. Mech. Bull., 47:3-4 (1982), 27-34; II, 37:3-4 (1982), 74-80; III, 38:3 (1983), 40-51; IV, 42:5 (1987), 40-49; V, 43:6 (1988), 23-29.

[52] В.В. Козлов, "Реализация неинтегрируемых связей в классической механике", Докл. АН СССР, 272:3 (1983), 550-554; англ. пер.: V. V. Kozlov, "Realization of nonintegrable constraints in classical mechanics", Soviet Phys. Dokl., 28:9 (1983), 735-737.

[53] V. V. Kozlov, "On the theory of integration of the equations of nonholonomic mechanics", Adv. in Mech., 8:3 (1985), 85-107.

[54] В.В. Козлов, “Линейные системы с квадратичным интегралом", ПММ, 56:6 (1992), 900-906; англ. пер.: V. V. Kozlov, "Linear systems with a quadratic integral", J. Appl. Math. Mech., 56:6 (1992), 803-809.

[55] В.В. Козлов, “Тензорные инварианты квазиоднородных систем дифференциальных уравнений и асимптотический метод Ковалевской-Ляпунова", Матем. заметки, 51:2 (1992), 46-52; англ. пер.: V. V. Kozlov, "Tensor invariants of quasihomogeneous systems of differential equations, and the Kovalevskaya-Lyapunov asymptotic method", Math. Notes, 51:2 (1992), 138-142.

[56] В. В. Козлов, “Лиувиллевость инвариантных мер вполне интегрируемых систем и уравнение Монжа-Ампера", Матем. заметки, 53:4 (1993), 45-52; англ. пер.: V. V. Kozlov, "The Liouville property of invariant measures of completely integrable systems and the Monge-Ampère equation", Math. Notes, 53:4 (1993), 389-393.

[57] В.В. Козлов, Методы качественного анализа в динамике твердого тела, 2-е изд., НИЦ "Регулярная и хаотическая динамика", М.-Ижевск, 2000, 248 с.; 1-е изд., Изд-во Моск. ун-та, М., 1980, 231 с.

[58] В. В. Козлов, Н. Н. Колесников, "О теоремах динамики", ПММ, 42:1 (1978), 28-33; англ. пер.: V.V. Kozlov, N. N. Kolesnikov, "On theorems of dynamics", J. Appl. Math. Mech., 42:1 (1978), 26-31.

[59] A.D. Lewis, R. M. Murray, "Variational principles for constrained systems: theory and experiment", Internat. J. Non-Linear Mech., 30:6 (1995), 793-815.

[60] A. Lichnerowicz, "Les variétés de Poisson et leurs algèbres de Lie associées", J. Differential Geom., 12:2 (1977), 253-300.

[61] В. Г. Марихин, В.В.Соколов, "О приведении пары квадратичных по импульсам гамильтонианов к канонической форме и о вещественном частичном разделении переменных для волчка Клебша", Нелинейная динам., 4:3 (2008), 313-332.

[62] C. M. Marle, "Various approaches to conservative and nonconservative nonholonomic systems", Rep. Math. Phys., 42:1-2 (1998), 211-229.

[63] C.-M. Marle, "A property of conformally Hamiltonian vector fields; application to the Kepler problem", J. Geom. Mech., 4:2 (2012), 181-206.

[64] R. Montgomery, A tour of subriemannian geometries, their geodesics and applications, Math. Surveys Monogr., 91, Amer. Math. Soc., Providence, RI, 2002, xx+259 pp.

[65] J. Moser, "On the volume elements on a manifold", Trans. Amer. Math. Soc., 120:2 (1965), 286-294.

[66] Н.К. Мощук, "О приведении уравнений движения некоторых неголономных систем Чаплыгина к форме уравнений Лагранжа и Гамильтона”, ПММ, 51:2 (1987), 223-229; англ. пер.: N. K. Moshchuk, "Reducing the equations of motion of certain non-holonomic Chaplygin systems to Lagrangian and Hamiltonian form", J. Appl. Math. Mech., 51:2 (1988), 172-177. 
[67] V. Narayanan, P. J. Morrison, Rank change in Poisson dynamical systems, 2013, 32 pp., arXiv: 1302.7267.

[68] Ю. И. Неймарк, Н. А. Фуфаев, Динамика неголономных систем, Наука, М., 1967, 520 с.; англ. пер.: Ju. I. Neimark, N. A. Fufaev, Dynamics of nonholonomic systems, Transl. Math. Monogr., 33, Amer. Math. Soc., Providence, RI, 1967, ix+518 pp.

[69] F. Noether, Über rollende Bewegung einer Kugel auf Rotationsflächen, K. B. LudwigMaximilians-Universität München, 1909, 56 pp.

[70] С. П. Новиков, "Гамильтонов формализм и многозначный аналог теории Морса", УМH, 37:5(227) (1982), 3-49; англ. пер.: S. P. Novikov, "The Hamiltonian formalism and a many-valued analogue of Morse theory", Russian Math. Surveys, 37:5 (1982), $1-56$.

[71] С.П. Новиков, И. Шмельцер, “Периодические решения уравнений Кирхгофа для свободного движения твердого тела в жидкости и расширенная теория Люстерника-Шнирельмана-Морса. 1", Функи. анализ и его прил., 15:3 (1981), 54-66; англ. пер.: S. P. Novikov, I. Shmel'tser, "Periodic solutions of Kirchhoff's equations for the free motion of a rigid body in a fluid and the extended theory of Lyusternik-Shnirel'man-Morse (LSM). I", Funct. Anal. Appl., 15:3 (1981), 197-207.

[72] С. П. Новиков, И. А. Тайманов, Современные геометрические структуры и поля, МЦНМО, М., 2005, 584 с.; англ. пер.: S. P. Novikov, I. A. Taimanov, Modern geometric structures and fields, Grad. Stud. Math., 71, Amer. Math. Soc., Providence, RI, 2006, xx+633 pp.

[73] T. Ohsawa, O.E. Fernandez, A. M. Bloch, D. V. Zenkov, "Nonholonomic Hamilton-Jacobi theory via Chaplygin Hamiltonization", J. Geom. Phys., 61:8 (2011), 1263-1291.

[74] A. Ramos, "Poisson structures for reduced non-holonomic systems", J. Phys. A, 37 (2004), 4821-4842.

[75] Э. Дж. Раус, Динамика системы твердых тел, I, II, Наука, М., 1983, 464 с., 544 с.; пер. с англ.: Е. J. Routh, The advanced part of a treatise on the dynamics of a system of rigid bodies, Being Part 2 of 'A treatise on the whole subject'. With numerous examples, 4th ed., revised and enlarged, MacMillan and Co., London, 1884, 343 pp.; Advanced dynamics of a system of rigid bodies, Dover Publications, New York, 1960, $496 \mathrm{pp}$.

[76] С. В. Станченко, "О неголономных системах Чаплыгина", ПММ, 53:1 (1989), 16-23; англ. пер.: S. V. Stanchenko, "Non-holonomic Chaplygin systems", J. Appl. Math. Mech., 53:1 (1989), 11-17.

[77] Г. К. Суслов, Теоретическая механика, Гостехиздат, М.-Л., 1946, 655 с.

[78] M. Svinin, A. Morinaga, M. Yamamoto, "On the dynamic model and motion planning for a spherical rolling robot actuated by orthogonal internal rotors", Regul. Chaotic Dyn., 18:1-2 (2013), 126-143.

[79] A. V. Tsiganov, "On two different bi-Hamiltonian structures for the Toda lattice", J. Phys. A, 40:24 (2007), 6395-6406.

[80] A. V. Tsiganov, "On bi-Hamiltonian geometry of the Lagrange top", J. Phys. A, 41:31 (2008), 315212, 12 pp.

[81] A. Tsiganov, "Integrable Euler top and nonholonomic Chaplygin ball", J. Geom. Mech., 3:3 (2011), 337-362.

[82] A.V. Tsiganov, "One invariant measure and different Poisson brackets for two non-holonomic systems", Regul. Chaotic Dyn., 17:1 (2012), 72-96.

[83] A.V. Tsiganov, "On the Poisson structures for the nonholonomic Chaplygin and Veselova problems", Regul. Chaotic Dyn., 17:5 (2012), 439-450.

[84] А. В. Цыганов, "Об одном семействе конформно-гамильтоновых систем", ТМФ, 173:2 (2012), 179-196; англ. пер.: A. V. Tsiganov, "One family of conformally Hamiltonian systems", Theoret. Math. Phys., 173:2 (2012), 1481-1497.

[85] А. В. Цыганов, "К задаче Чаплыгина о качении шара", Матем. заметки, 94:4 (2013), 637-640; англ. пер.: A. V. Tsiganov, "On the Chaplygin problem of the rolling of a ball", Math. Notes, 94:4 (2013), 599-602. 
[86] В. В. Вагнер, "Геометрическая интерпретация движения неголономных динамических систем", Тр. семинара по векторному и тензорному анализу, 5, МГУ, М., $1941,301-327$.

[87] A. J. van der Schaft, B. M. Maschke, "On the Hamiltonian formulation of nonholonomic mechanical systems", Rep. Math. Phys., 34:2 (1994), 225-233.

[88] А. М. Вершик, Л.Д. Фаддеев, "Дифференциальная геометрия и лагранжева механика со связями”, Докл. АН СCCP, 202 (1972), 555-557; англ. пер.: A. M. Vershik, L. D. Faddeev, "Differential geometry and Lagrangian mechanics with constraints", Soviet Phys. Dokl., 17 (1972), 34-36.

[89] А. М. Вершик, Л. Д. Фаддеев, "Классическая и неклассическая динамика со связями", Геометрия и топология в глобальных нелинейных задачах, Новое в глобальном анализе, Изд-во Воронеж. гос. ун-та, Воронеж, 1984, 23-48.

[90] А. М. Вершик, В. Я. Гершкович, "Неголономные задачи и геометрии распределений”, прил. к кн.: Ф. Гриффитс, Внешние дифференциалъные системъ и вариаиионное исчисление, Мир, М., 1986, 318-349.

[91] А. М. Вершик, В. Я. Гершкович, "Неголономные динамические системы. Геометрия распределений и вариационные задачи", Динамические системы - 7, Итоги науки и техники. Сер. Соврем. пробл. матем. Фундам. напр., 16, ВИНИТИ, M., 1987, 5-85; англ. пер.: A. M. Vershik, V. Ya. Gershkovich, "Nonholonomic dynamical systems. Geometry of distributions and variational problems", Dynamical systems, VII, Encyclopaedia Math. Sci., 16, Springer-Verlag, Berlin, 1994, 1-81.

[92] А.П. Веселов, Л.Е. Веселова, "Интегрируемые неголономные системы на группах Ли", Матем. заметки, 44:5 (1988), 604-619; англ. пер.: А.P. Veselov, L. E. Veselova, "Integrable nonholonomic systems on Lie groups", Math. Notes, 44:5 (1988), 810-819.

[93] Л.Е. Веселова, “Новые случаи интегрируемости уравнений движения твердого тела при наличии неголономной связи", Геометрия, дифференииалъные уравнения и механика (Москва, 1985), МГУ, 1986, 64-68.

[94] П.В. Воронец, К задаче о движении твердого тела, катящегося без скольжения по данной поверхности под действием данных сил, Предварит. сообщ., чит. в заседании Физ.-мат. о-ва при Ун-те св. Владимира 23 февр. 1909 г., Тип. Имп. Ун-та Св. Владимира, Киев, 1909, 11 с.

[95] D. V. Zenkov, "The geometry of the Routh problem", J. Nonlinear Sci., 5:6 (1995), 503-519.

\section{Алексей Владимирович Борисов}

Удмуртский государственный университет

E-mail: borisov@rcd.ru

\section{Иван Сергеевич Мамаев}

(Ivan S. Mamaev)

Удмуртский государственный университет;

Ижевский государственный технический

университет им. М. Т. Калашникова

E-mail: mamaev@rcd.ru

\section{Андрей Владимирович Цыганов (Andrey V. Tsiganov)}

Санкт-Петербургский государственный университет

E-mail: andrey.tsiganov@gmail.com 